Portland State University

PDXScholar

$2-22-1980$

\title{
The Effect of Residual Stress Distribution on the Ultimate Strength of Tubular Beam-Columns
}

Steven L. Barrett

Portland State University

Follow this and additional works at: https://pdxscholar.library.pdx.edu/open_access_etds

Part of the Civil Engineering Commons

Let us know how access to this document benefits you.

\section{Recommended Citation}

Barrett, Steven L., "The Effect of Residual Stress Distribution on the Ultimate Strength of Tubular BeamColumns" (1980). Dissertations and Theses. Paper 3050.

https://doi.org/10.15760/etd.3045

This Thesis is brought to you for free and open access. It has been accepted for inclusion in Dissertations and Theses by an authorized administrator of PDXScholar. Please contact us if we can make this document more accessible: pdxscholar@pdx.edu. 
AN ABSTRACT OF THE THESIS OF Steven L. Barrett for the Master of Science in Applied science presented February 22, 1980.

Title: The Effect of Residual stress Distribution on the Ultimate strength of Tubular Beam-Columns.

APPROVED BY MEMBERS OF THE THESIS COMMITTEE:

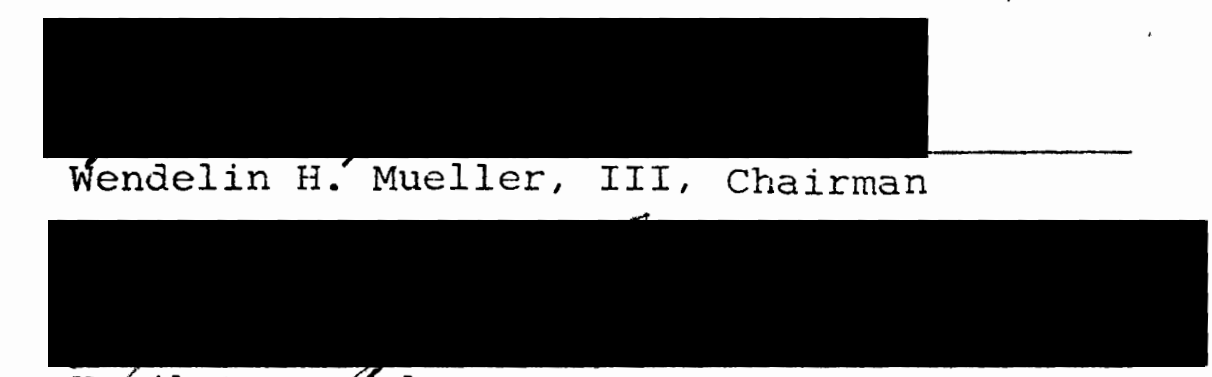

Hacik Erzugfomlu

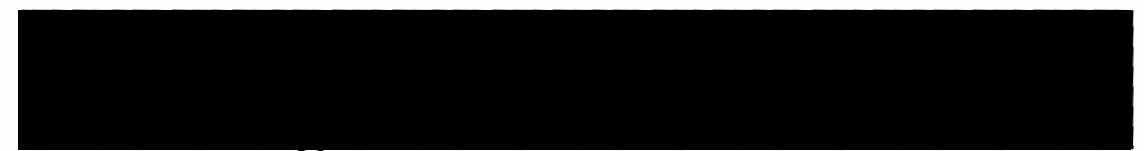

Ansel Jabsinson

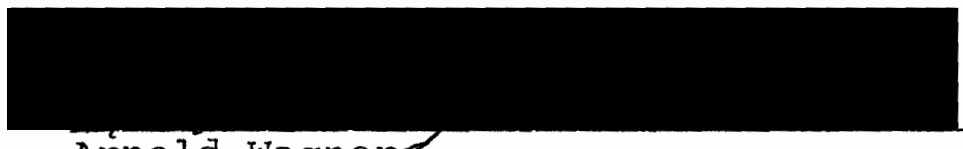

Arnold Wagnerd

Using data for the longitudinal residual stress distribution in welded steel tubes, curves describing these distributions are selected for study. Each of these curves are checkea for static balance across the tube cross section. The curves that exhibit an imbalance are adjusted by a combination of a simplified model for each 
and the use of a computer program that is developed to calculate the resulting forces and moments on the cross section. The residual stress in the area of the tube wall opposite the longitudinal weld is found to be the most important in the adjustment to obtain exact equilibrium. The method of adjustment is rational and based on maintaining a smooth curve shape that matches the raw data the closest and producing a curve that is balanced within the accuracy limits required.

The balanced longitudinal residual stress distributions are used to determine M-P- $\varnothing$ relationships, where $M$ equals moment, $P$ equals axial load, and $\varnothing$ equals curvature. A computer program developed by Wagner (13) is modified to determine these relationships. The program was originally developed for a bending axis through the weld and the results are found to be inaccurate for other orientations. The problem is identified as the creation of a moment on the cross section from an uneven distribution of axial stress caused during the iteration to determine axial strain from an applied axial force. The problem is solved by adding another iteration to the program to redistribute the axial strains and eliminate the moment.

The M-P- $\varnothing$, relationships obtained from this computer program for three longitudinal residual stress distributions with five different bending axis orientations are compared and one residual stress distribution curve is selected 
to compare with Wagner's (13) distribution. The beam column failure load computer program (13) is used with the $\mathrm{M}-\mathrm{P}-\varnothing$ relationships generated and the results are compared with test data.

The conclusion is reached that residual stresses have a large influence on the behavior of welded steel tube beam columns. The difference in effect of each residual stress distribution is not large. This could be because all of the curves have the same general shape with very large stresses close to the weld and rapidly decreasing away from it. With the effect of residual stresses included, the beam column failure load program still overestimates actual member strength. Therefore, other conditions effecting member strength when used as beam columns require further study and the residual stress distribution labeled curve number three which is Chen and Ross's $(3,4)$ curve forced to two maximums from $\mathrm{x} / \mathrm{d}=1.0$ to $\mathrm{x} / \mathrm{d}=\pi$ is recommended for use in further research. 
THE EFFECT OF RESIDUAL STRESS

DISTRIBUTION ON THE ULTIMATE STRENGTH

$\mathrm{OF}$

TUBULAR BEAM-COLUMNS

by

STEVEN L. BARRETT

A thesis submitted in partial fulfillment of the requirements for the degree of

MASTER OF SCIENCE

in

APPLIED SCIENCE

Portland State University

February 1980 
TO THE OFFICE OF GRADUATE STUDIES AND RESEARCH:

The members of the Committee approve the thesis

of Steven L. Barrett presented February 22, 1980.
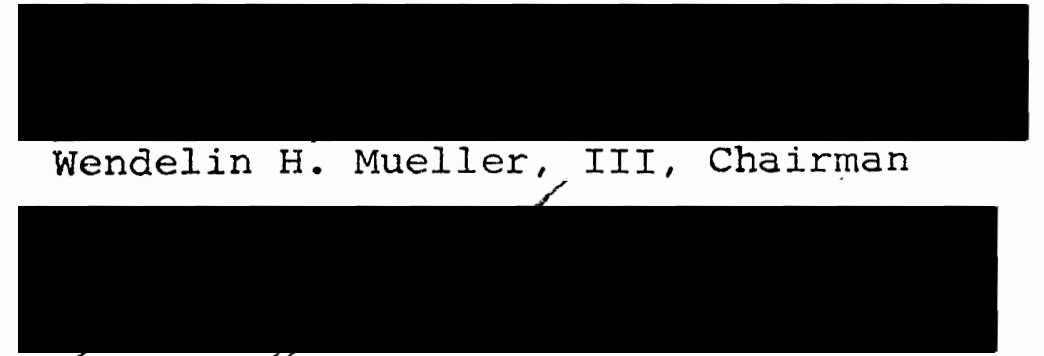

Kacik Exźurumiu
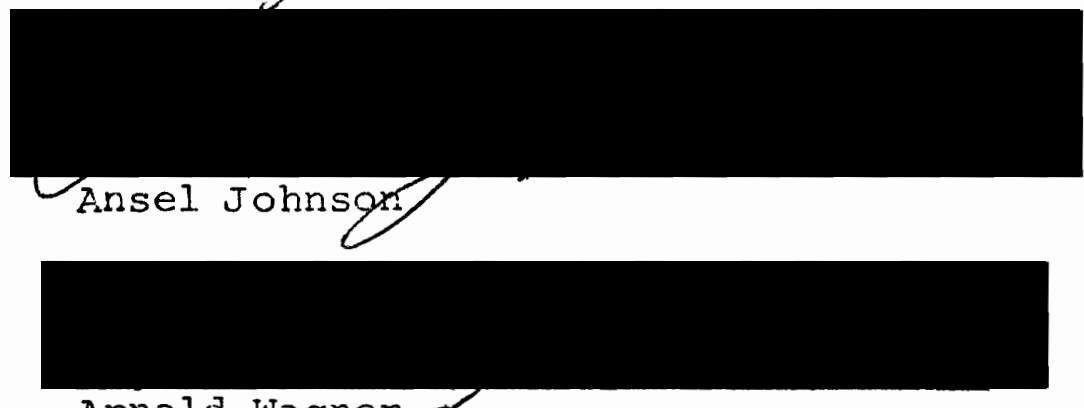

APPROVED:

Arnola Wagner

Frañ N. Rad, Gead, Civil-Structural Engineering

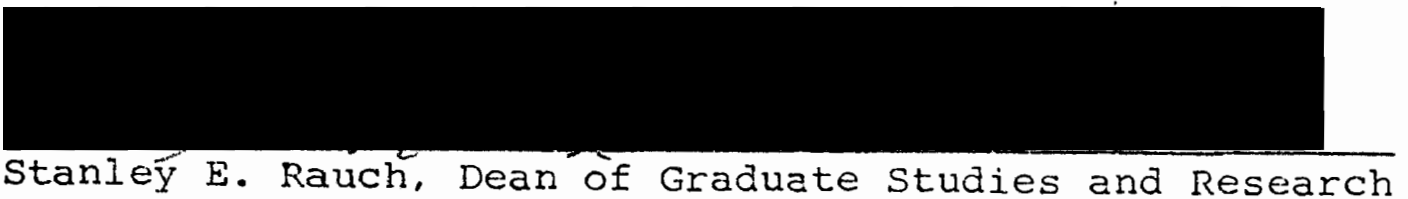


TABLE OF CONTENTS

PAGE

LIST OF FIGURES

CHAPTER

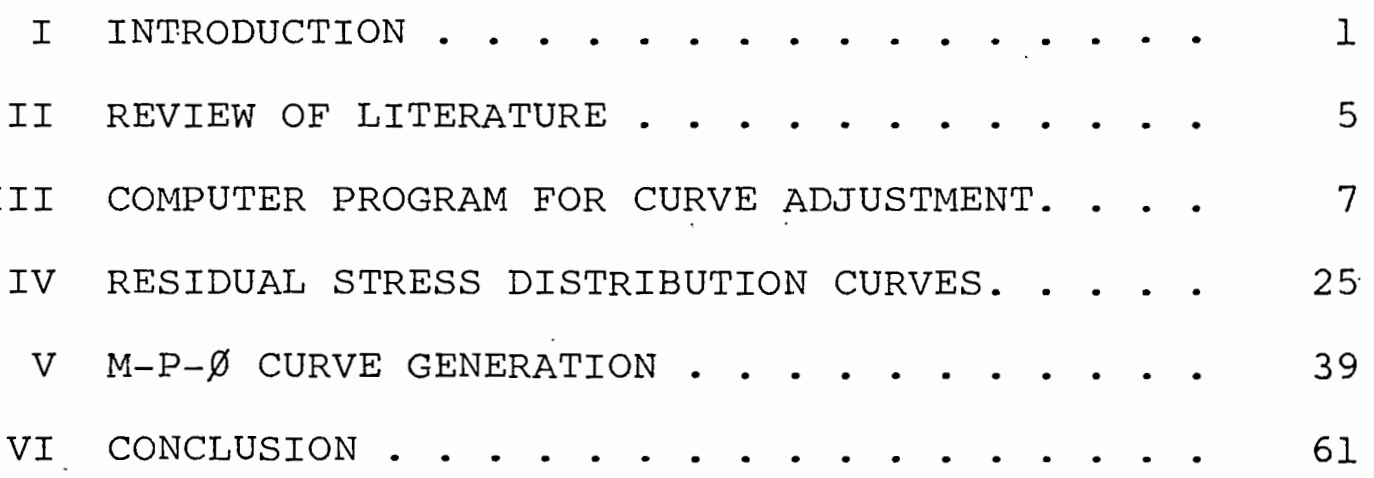

REFERENCES . • • • • • • • • • • • • • • • • • • • 
LIST OF FIGURES

FIGURE

PAGE

1. Longitudinal Residual Stress in a Welded Steel Tube . . . . . . . . . . . . . 9 9

2. Longitudinal Residual Stress . . . . . . . 9

3. Area Under Curve . . . . . . . . . . . . 11

4. Arc Length - Lever Arm . . . . . . . . . . 11

5. Areas Under Curve. . . . . . . . . . . . 13

6. Areas Under Curve. . . . . . . . . . . . 14

7. Flow Chart - Moment, Force Summation . . . . 15

8. Residual Stress From Tran (II) . . . . . . 18

9. Approximate Curve. . . . . . . . . . . . 21

10. Approximate Curve. . . . . . . . . . . 22

11. Residual Stress $\operatorname{Tran}(11)$. . . . . . . . 26

12. Simplified Curve . . . • • . • • • • . . 27

13. Residual Stress Chen \& Ross $(3,4)$. . . . . 29

14. Residual Stress, Balanced Chen \& Ross $(3,4)$ Curve 1. • • • • . . • • . • . . . 31

15. Residual Stress First Section Average - Chen. \& Ross $(3,4)-\operatorname{Tran}(I 1) \quad$ Curve 2. . . . 32 16. Fluctuation in Curve shape . . . . . . . . 33

17. Residual' Stress First Portion, Smoothed Curve from $\operatorname{Tran}(11)$. . . . . . . . . . 
18. Residual Stress Chen \& Ross $(3,4)$ Forced to

Two Maximums Curve 3. . . . . . .

19. Flow Diagram for Calculation of M-P- $\varnothing$ Data

(Wagner) ............ . . 40

20. Orientation of Axes and Residual Stress

Distribution (Wagner $(12,13)$. . . . 44

21. M-P- $\varnothing$ Curve 1 (Chen \& Ross)......... . 46

22. M-P- $\varnothing$ Curve 2 (First Section Average). . . . 47

23. M-P- $\varnothing$ Curve 3 (Chen \& Ross Forced To Two

Maximums). . . . . . . . . . 48

24. Moment Imbalance .. . . . . . . . . 53

25. M-P- $\varnothing$ Curves From Improved Generation Program. 56

26. Beam Column Results - Wagner's Distribution. • 59

27. Beam Column Results - Curve 3 (Fig. 18). . . 60

28. Longitudinal Residual Stress Distribution

Curves ............. . . 62

29. Moment Curvature Relationships . . . . . 63

30. Effect of Residual Stress on Strength Under

Axial Load . . . . . . . . . . 64

31. Effect of Residual Stress .......... 65

32. Example for Testing Curve Program. . . ... 81 
CHAPTER I

INTRODUCTION

The idealized model that is used to exhibit the behavior of steel in most mechanics of materials texts has well defined characteristics. The idealized material is stress-strain free prior to application of any load, there are no thermal strains, the material is homogenous and it has a bilinear stress strain relationship with constant slope to yield and a constant value beyond. If this material is used in an idealized member it is possible to theoretically predict the member's exact behavior under load. For different cross section shapes it is possible to determine the effect of slenderness on the member."s behavior if it is used as a compression member, a beam, or a beam-column.

For a real structural member the prediction of actual behavior is much more difficult. Actual materials are not entirely homogenous and do not have bilinear stress strain curves. A real structural member is not stress free prior to loading, may have a complex cross section shape which has, no simple closed form solution for critical buckling load, may have accidental eccentricity of load, initial crookedness and any number of stresses 
induced during erection. Each of these conditions that can be accurately measured in the laboratory should be investigated to determine its individual effect on the strength of the member. Once the relative magnitude of the individual effect is known, a decision can be made whether or not to include that effect in every member analysis. To that end, there has been considerable research done on certain structural shapes in various materials. The largest volume of research has been done on rolled steel wide flange shapes. due to their widespread application for structures of all types. $(1,2,5,8,10)$

Because the information available for the steel wide flange shape is extensive, there has been a tendency to apply it to other cross section shapes where data has not yet been developed. For any particular case this practice may or may not be conservative. It is not possible to completely discount any of the previously mentioned effects on a cross section shape other than a wide flange based on the argument that such an effect did not alter the behavior of the wide flange as idealized. Nor is it possible to make a direct comparison without some data being developed for the particular cross section.

The complete description of the effect of all possible initial conditions on a welded steel tube cross section for all types of loading is too broad in scope for this study. Therefore the single consideration of the effect of residual 
stress was selected to study. Residual stresses are those stresses which are present in a member after it is fabricated to its finished form. Usually these residual stresses cause a reduction in strength in fatigue, fracture and stability although for some distributions a strengthening at certain load levels will be exhibited. These stresses may result from uneven cooling after hot rolling as for structural shapes and plates, from cold bending during fabrication, from welding or from cutting, drilling and punching during fabrication. Usually residual stresses with the largest magnitude result from cooling and welding. In many cases, residual stresses in the region of a weld will be greater than the yield stress. It therefore becomes important to determine the distribution and magnitude of residual stress in a member that has extensive welding during fabrication. Recent research has suggested some possible distributions of both longitudinal and circumferential residual stresses in welded steel tubes. $(3,7,11,12,13)$

The primary goal of this project was to analyze and select one longitudinal residual stress distribution in welded steel tubes from the literature and test data available and then determine the effect of this distribution on the ultimate beam-column failure load of the tube. The circumferential residual stress was not included since the project was simplified excluding the effects of 
local buckling or ovaling from consideration. After determination of the residual stress distribution, momentcurvature-axial load curves could be generated by computer. These describe the performance of the cross section of the tube. Then, using these curves, an iterative open form computer solution calculates the beam column failure load. The solution obtained is compared to the ideal case of no residual stress and with the results of previous research using different distributions. From this comparison it is possible to determine the relative effect of residual stresses in determining failure load and the effect of changing the distribution. 
CHAPTER II

REVIEW OF LITERATURE

The distribution of longitudinal residual stress described by a linear variation between three peak values was used by Wagner $(12,13)$ in the absence of actual test data. This distribution gave a starting point for the computation of $\mathrm{M}-\mathrm{P}-\varnothing$ curves, where M equals moment, $\mathrm{P}$ equals axial load and $\varnothing$ equals curvature with the effect of some residual stress added. A computer program was developed using the assumed general shape that could adjust the value of residual stress on each element of the tube such that force and rotational equilibrium would be satisfied.

While there is a large volume of information on the magnitude and distribution of residual stresses in wide flange sections $(4,8)$, there is a scarcity of information about the distribution and magnitude in welded steel tubes or its effect on the beam column failure load. Some investigators have suggested possible theoretical distributions (7) while others (4) have attempted to use more realistic patterns in correlating experimental results. They, however, discounted the necessity of static equilibrium across the cross section considering the imbalance of 
internal forces to be of little consequence. Further, no consideration of the uniformity of the stress field through the tube wall thickness was made.

Recently however Tran (11) developed test data that first confirmed a uniform field of longitudinal stress through the wall of a tube and showed a very close approximation to static equilibrium across the section. 
In order to determine the best curve to describe the longitudinal residual stress distribution in the welded tube it was necessary to generate a method of rapidly checking the balance of forces and moments that each distribution curve implied. If the residual stress distribution through the thickness of the tube wall was not uniform, it would be necessary to check the balance for several layers and use that information in the generation of moment-curvature axial load curves. However, Tran (11) has shown that the distribution through the tube wall is uniform. Therefore, it is only necessary to consider one layer equal to the tube wall thickness. To check the balance of forces and moments accurately and quickly, it was decided to write a computer program that could handle any general curve describing the residual stress pattern.

Consider the general longitudinal residual stress distribution shown in Figure 1. The ordinates of the residual stresses a re measured along the $\mathrm{x}$ axis with the weld located on the $Y$ axis. To make the curve easier to represent, it is convenient to straighten the circumference 
of the tube, in effect unrolling the tube so it is flat (Fig. 2). Now the area under any portion of this curve will represent a force, since the distance along the circumference multiplied by the wall thickness is area. Area multiplied by stress, which is force per unit area, is force. This force wili be located at the center of gravity of the area under the stress curve. The distance from any single point on the tube circumference to the force, multiplied by the force will equal moment. Since the tube is under no external loading and therefore must be in static equilibrium for internal stresses, the summation of forces and the summation of moments over the entire cross section of the tube must be equal to zero.

In general the problem is to find the area (force), center of gravity and first moment of area (moment) about some point for segments under a general curve that the mathematical description for is not known. The method used will be a linear approximation of segments of the curve, in other words, short straight lines between points on the curve. Sign convention used for this development were: Tensile stresses positive, plotted above the $\mathrm{X}$ axis (Fig. 2); compressive stresses negative, plotted below the $\mathrm{X}$ axis; positive moment about any singular point, clockwise; negative moment counter clockwise. There are several possible conditions that must be accounted for if the program is to handle all possible 


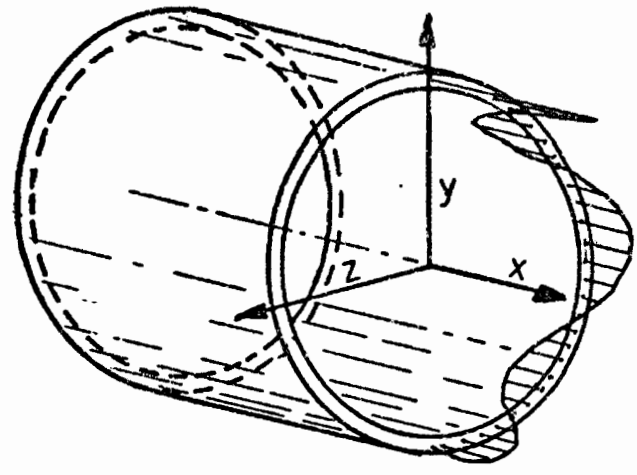

Figure 1. Longitudinal residual stress in a welded steel tube

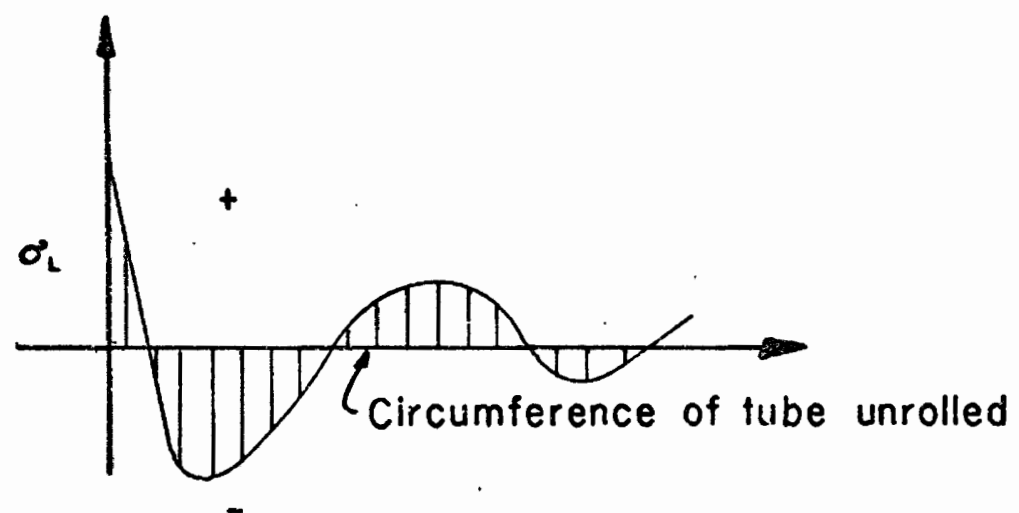

Figure 2. Longitudinal residual stress 
Refering to Figure 3:

Let $\mathrm{Y}_{1}=$ First ordinate on curve

$$
\begin{aligned}
& \mathrm{Y}_{2}=\text { Second ordinate on curve } \\
& \mathrm{X}_{1}=\text { First abscissa on curve } \\
& \mathrm{X}_{2}=\text { second abscissa on curve }
\end{aligned}
$$

C.G. ${ }_{1}=$ - Distance from $Y$ axis to center of gravity of area

C.G. 2 = Distance from $Y$ axis to center of gravity of area 2

$$
\text { Area }_{1}=\left(X_{2}-X_{1}\right)\left(Y_{1}\right)
$$$$
\text { C.G. } 1=\frac{\left(x_{2}-x_{1}\right)}{2}+x_{1}=\frac{x_{2}-x_{1}+2 x_{1}}{2}=\frac{x_{1}+x_{2}}{2}
$$

$$
\text { Area }_{2}=\frac{\left(X_{2}-X_{1}\right)\left(Y_{2}-Y_{1}\right)}{2}
$$

C.G. $2=2 / 3\left(x_{2}-x_{1}\right)+x_{1}=\frac{2 x_{2}-2 x_{1}+3 x_{1}}{3}=\frac{2 x_{2}+x_{1}}{3}$

Now since this is for a tube the $\mathrm{x}$ distances can be thought of as arc lengths. (Fig. 4)

C.G. x $_{\mathrm{x}}=$ Arc Length

$\frac{1}{2}$ circumference $=\frac{2 \pi r}{2}=\pi r$

$\theta($ radians $\left.)=\frac{(C \cdot G \cdot x)}{(\pi r}\right) \frac{(2 \pi)}{(2)}=\frac{C \cdot G \cdot x}{r}$

Lever $\mathrm{arm}=\mathrm{L}=\mathrm{r}-(\cos \theta) r=(1-\cos \theta) r$

Moment $=$ (Force) (Lever Arm)

Force $=$ Area, Lever Arm $=\mathrm{L}$

$M=\left(F_{1}\right)\left(I_{1}\right)+\left(F_{2} \cdot L_{2}\right)$

$M=\left(\right.$ Area $\left._{1}\right)\left(r-\operatorname{Cos} \frac{\left(\mathrm{C} \cdot \mathrm{G}_{1}\right)}{r}+\left(\operatorname{Area}_{2}\right)\left(r-\operatorname{Cos} \frac{\left(\mathrm{C} \cdot \mathrm{G}_{2}\right)}{r}\right)\right.$

$$
\begin{gathered}
M=\left[\left(X_{2}-X_{1}\right)\left(Y_{1}\right)\right]\left[1-\cos \frac{\left(X_{1}+X_{2}\right)}{2 r}\right](r)+\left[\frac{\left(2 X_{2}+X_{1}\right)}{3}\right. \\
\left.\left(Y_{2}-Y_{1}\right)\right]\left[I-\cos \frac{\left(2 X_{2}+X_{1}\right)}{r}\right](r)
\end{gathered}
$$




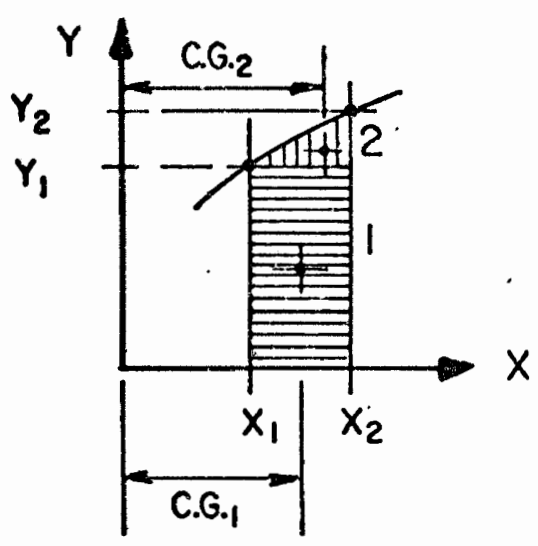

Figure 3. Area under curve
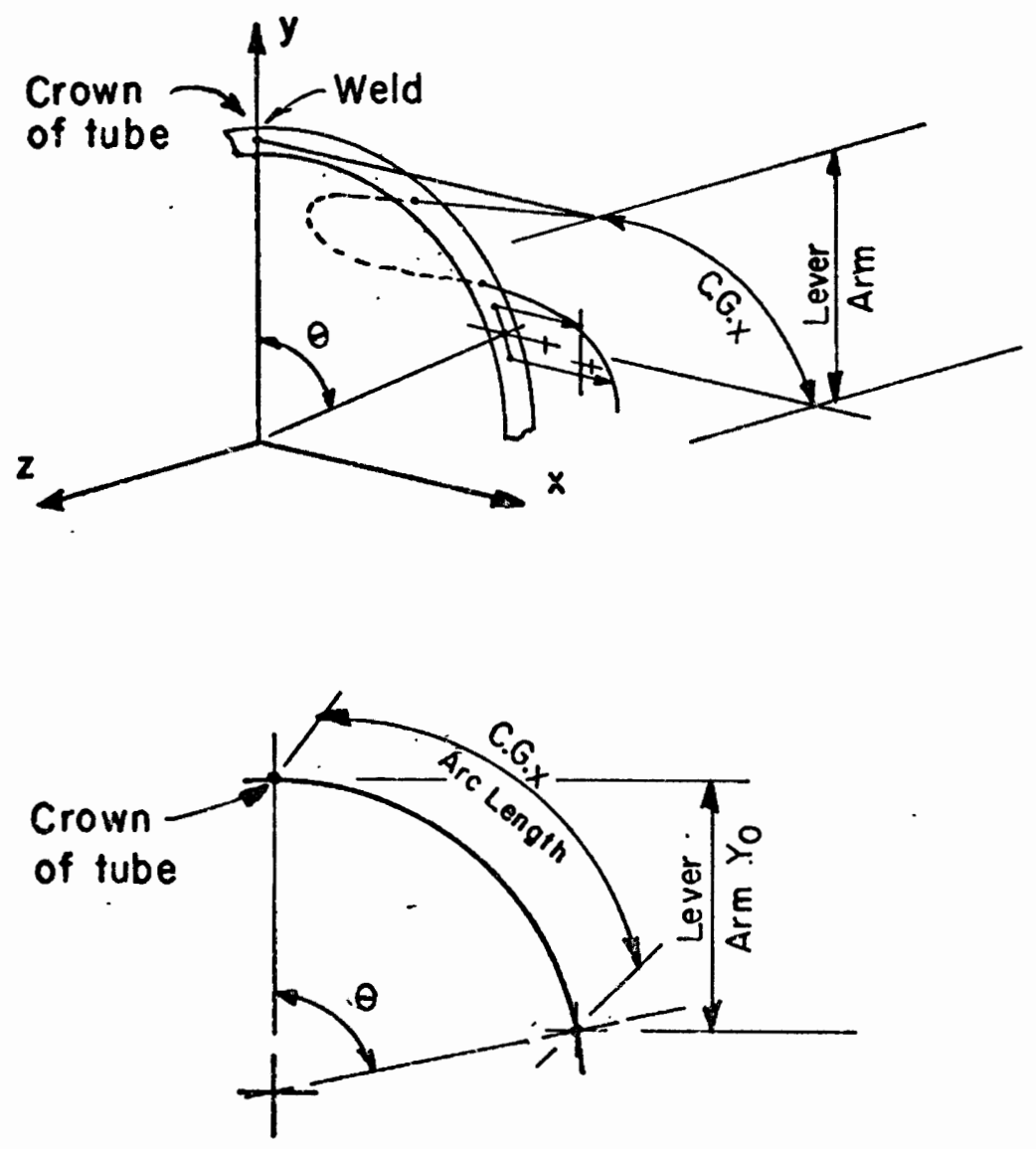

Figure 4. Arc length - Lever arm 
cases. of slope of the curve and axis interception (Fig. $5 \& 6)$. Taking each of these one at a time, we can express each area and center of gravity in the same terms used previously (Fig. 6). The location of the center of gravity for case 5 and 2 are equivalent (for the $\mathrm{x}$ direction) as are case 6 and 1 as well as case 3 and 4 . Thinking of this in flow chart form, we have the central part of the force-moment program complete (Fig. 7). Starting with the coordinates of a known curve, it is possible to sum the forces and moments for all the segments of the curve and arrive at a total that will indicate whether or not the section is in static equilibrium. The method could be used for random lengths of curve segments or for a constant increment. The program used for this project used a constant increment for the abscissa simplifying the input required since the ordinates become the only required input. These ordinates were scaled from each curve selected for analysis. Since the summations should be zero about any point, the crown of tube was selected for convenience.

Assuming that an imbalance of force is discovered, it is possible to select the largest ordinate, divide by 10.0 and use this value to modify all ordinates until a change of sign of the summation of forces is detected. Reducing the increment applied to the ordinates, it is possible to continue the iteration until a force balance 

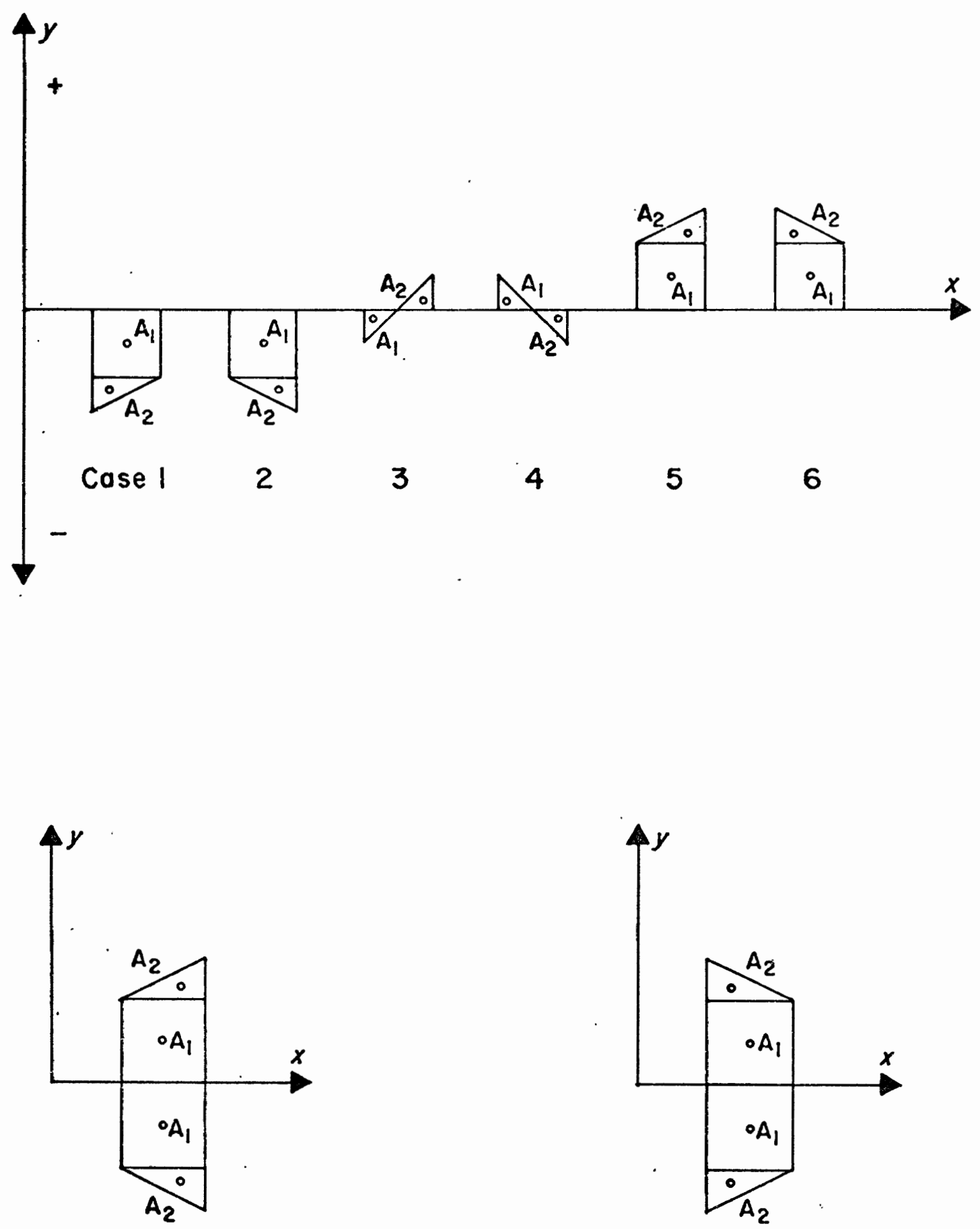

Case $2 \& 5$

Case $1 \& 6$ 


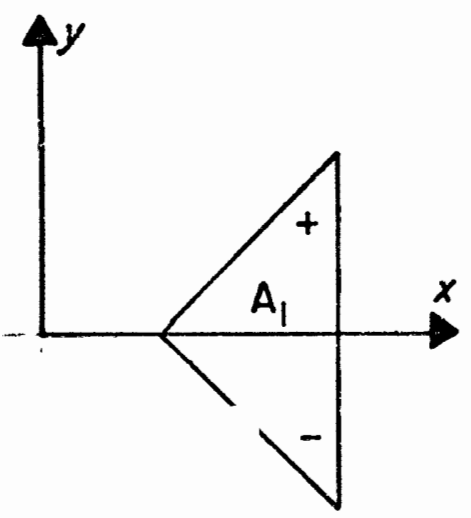

$$
\begin{aligned}
& A_{1}=\frac{\left(x_{2}-x_{1}\right)\left(y_{2}\right)}{2} \\
& C G=\frac{2\left(x_{2}-x_{1}\right)}{3}+x_{1}=\frac{2 x_{2}+x_{1}}{3}
\end{aligned}
$$

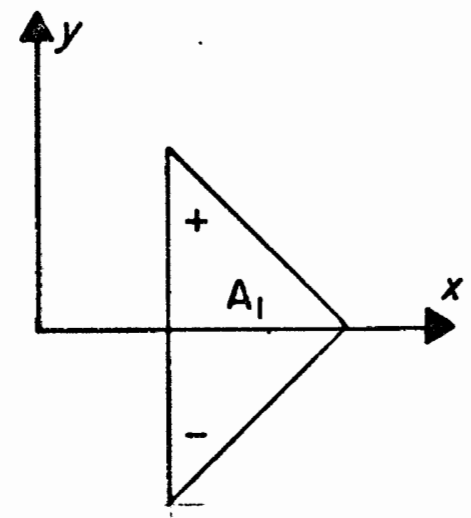

$$
A_{1}=\frac{\left(x_{2}-x_{1}\right)\left(y_{1}\right)}{2}
$$

$C G=\frac{\left(x_{2}-x_{1}\right)}{3}+x_{1}=\frac{x_{2}+2 x_{1}}{3}$

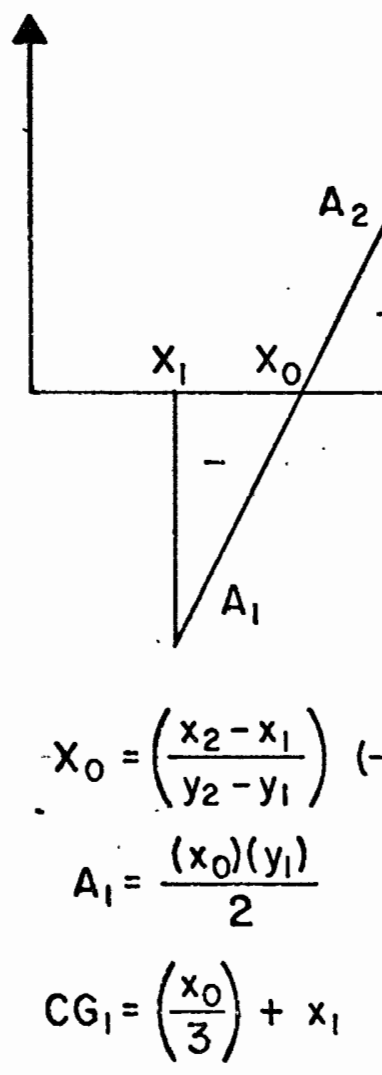

$\left(-y_{1}\right)$

$$
\begin{aligned}
& A_{1}=\frac{\left(x_{0}\right)\left(y_{1}\right)}{2} \\
& A_{2}=\frac{\left[x_{2}-\left(x_{1}+x_{0}\right)\right]\left(y_{2}\right)}{2} \\
& C G_{2}=\frac{2\left[\left(x_{2}-x_{1}\right)-x_{0}\right]}{3}+\left(x_{1}+x_{0}\right) \\
& =\frac{2 x_{2}-2 x_{1}-2 x_{0}+3 x_{1}+3 x_{0}}{3} \\
& =\frac{2 x_{2}+x_{1}+x_{0}}{3}
\end{aligned}
$$

Figure 5. Areas under curve 


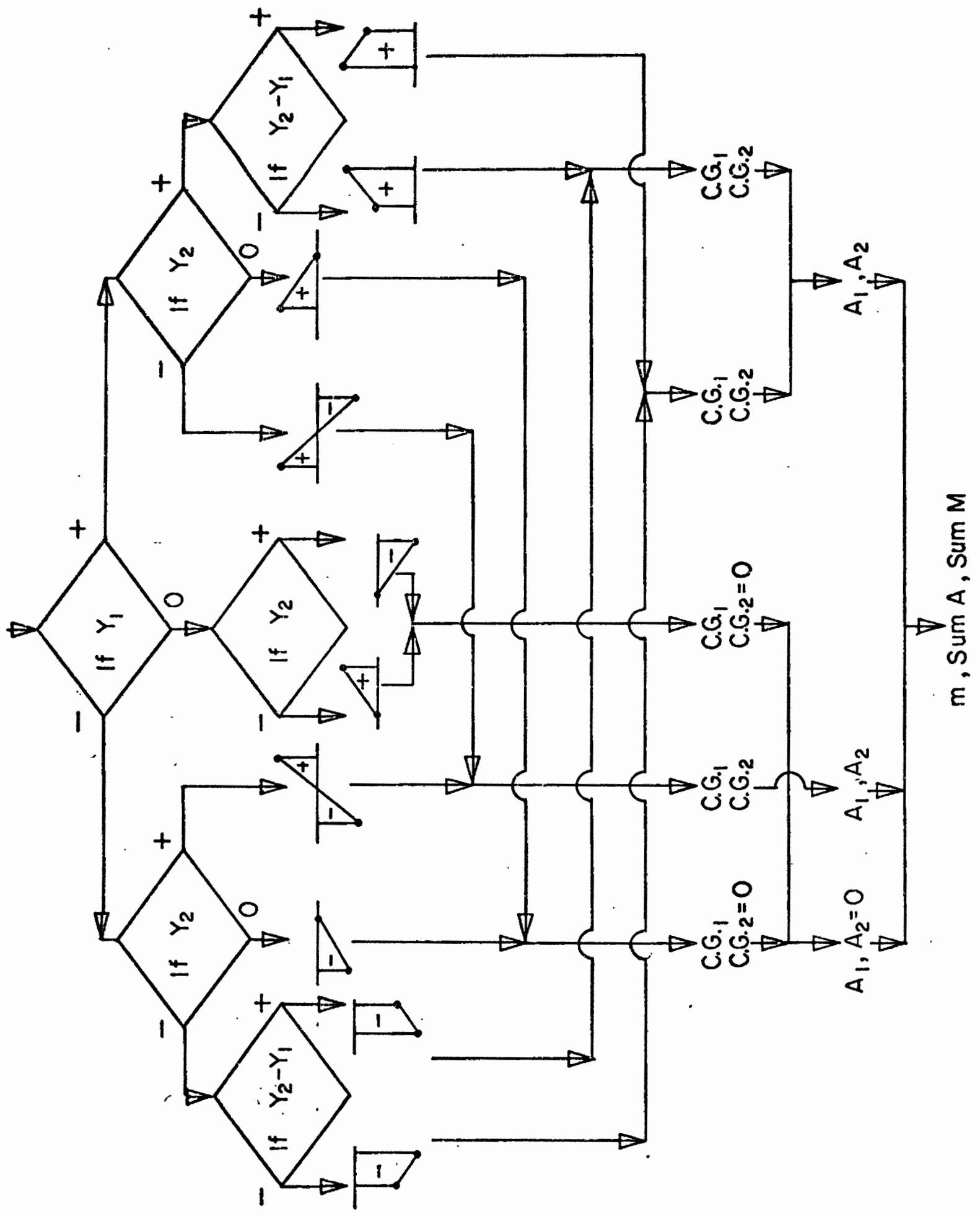

Figure 7. Flow chart-Moment, force summation 
of zero is obtained. The result of this procedure is a shift of the $\mathrm{X}$-Axis of the curve. Re-entering the balancing program, the balance of moment for the new ordinates can be found. Now if the moment is not balanced, the program becomes how to select the location and amount of change required to preserve force balance, the general curve shape and bring about moment equilibrium.

In order to maintain a smooth curve, the changes made to balance the moment should vary from ordinate to ordinate. With a large number of ordinates the changes possible become large and the number of possible solutions increases. Because the balanced curve should be the same general shape as the curve started with, each possible solution would have to be compared with it and measured against some criteria to find the best solution. Rather than design a program to do that it was decided to first find a solution by simplifying each curve, approximating the areas, and doing the balancing calculations by hand. This way possible curve shapes could be evaluated by determining the balanced curve ordinates and comparing them with reasonable stress values at each element, thereby eliminating curves with impossible or unlikely distributions. Then using the more exact summation program previously described on each selected curve, the imbalance for moment and force could be determined. Using this information, changes could be made to the curve and the summation pro- 
gram run again. This method eliminates some cases and within four to six runs through the summation program it is possible to find a solution that satisfies the accuracy limit required by Wagner's (13) M-P- $\varnothing$ Program.

As one example, the curve suggested by Tran (11) as shown in Figure 8 might be approximated as shown in Figure 9. The summation of forces and moments about the weld can be done as follows:

$d=$ Diameter $\quad \propto=$ Angle from weld to center of gravity $\mathrm{x}=\operatorname{Arc}$ length to C.G. L.A. = Lever arm to center of gravity $r=$ Radius to center line of element layer $=0.9635 \mathrm{in}$. $\frac{x}{d}=0.1 \quad x=0.1$ radians $L \cdot A=(1-\cos \alpha) r=0.005$ in. $\frac{x}{d}=0.3+0.35=0.65 \alpha=0.65$ radians $L . A .=0.184$ in. $\frac{x}{d}=1.00+2.14 / 2=2.071 \alpha=2.071$ radians $L . A .=1.337$ $\Sigma F=\left(\frac{1}{2}\right)(0.30)(4.7)-(0.7)(4.57)+(1.61)(2.14)$ $=0.70-3.20+3.45=+0.95$ $\Sigma M=+0.70(0.005)-3.20(0.184)+3.45(1.337)$ $=0.335-0.589+3.343=+4.057$

The points Tran's (11) data gives generate a curve with an imbalance of positive force and positive moment. The imbalance is perhaps within tolerable experimental error for the data gathered $(0.02 \mathrm{Py}$ and $0.027 \mathrm{My}$ where Py equals axial load at yield, My equals moment at yield) but the accuracy of balance for use of the residual stress 


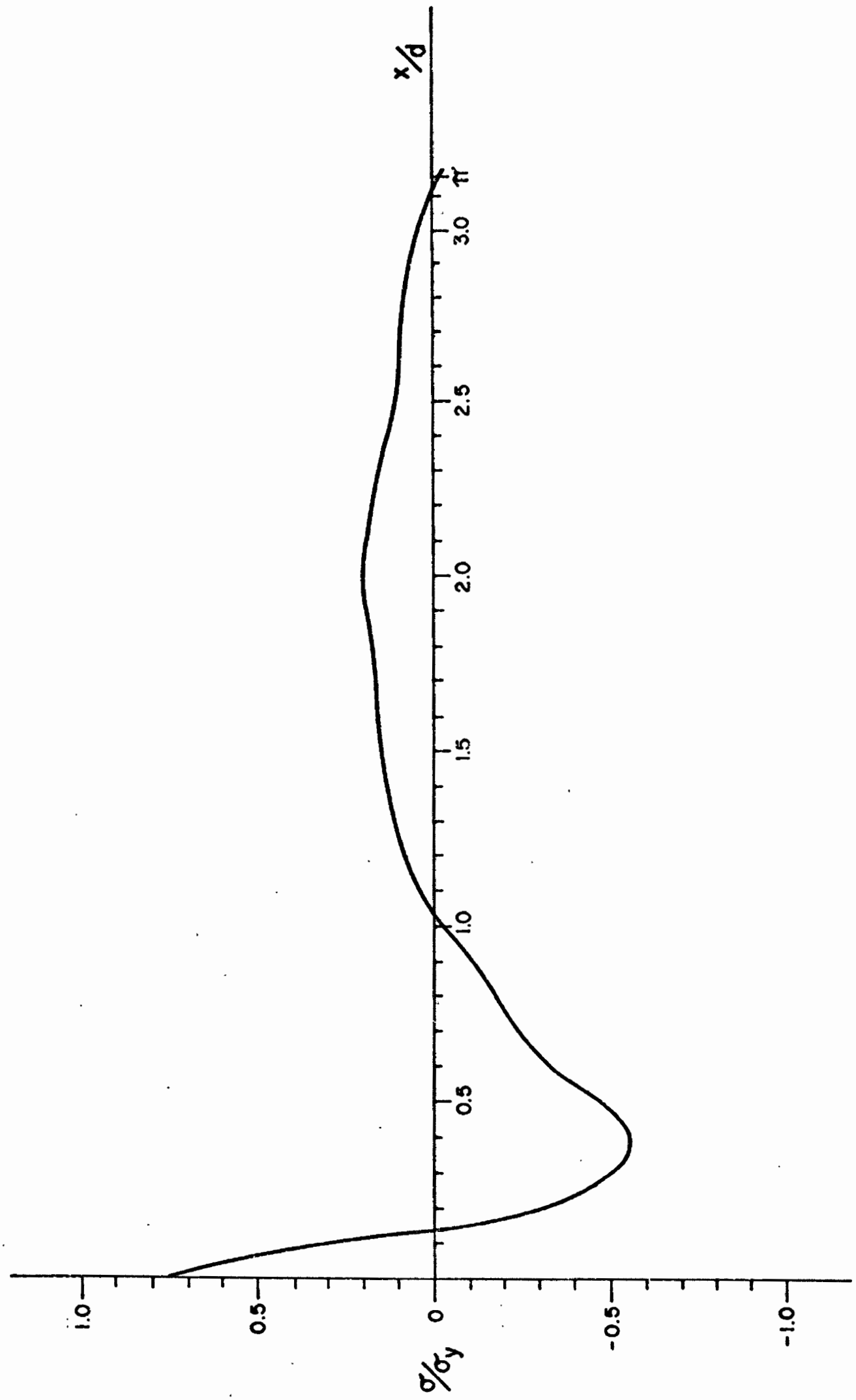

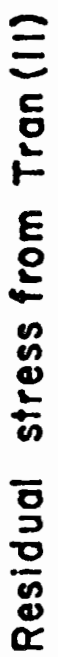
$\infty$
0
2
$\frac{2}{2}$
$\frac{0}{4}$ 
distribution in the $\mathrm{M}-\mathrm{P}-\varnothing$ program are much more restrictive $(0.00005 \mathrm{Py}$ and $0.001 \mathrm{My})$. Comparing the results of the balancing program for Tran's (11) curve and the approximate solution, close agreement is found confirming the computer code. A complete check of the program is given in Appendix I. Using the simple approximation, it can be shown that changing the portion of the curve from $\mathrm{x} / \mathrm{d}=1.0$ to $\mathrm{x} / \mathrm{d}=\pi$ so that the summation of forces is zero still leaves a positive unbalanced moment.

What then can be done to develop a curve based on Tran's (11) data that will satisfy $\Sigma F=0 \& \Sigma M=0$ ? The first portion (to $\mathrm{x} / \mathrm{d}=1.0$ ) of $\operatorname{Tran}(11)$ \& Chen-Ross (4) curves are of the same shape although of different amplitude and therefore, a modification of the portion of the curve from $x / d=1.0$ to $x / d=\pi$ seemed to be a good possibility. This is the area that previous investigators have regarded as being of lesser importance probably due to the rapidly decreasing magnitude of residual stress; although it is the most important area for accomplishing static equilibrium.

On viewing the simplified model, it is quite apparent that the positive area past $\mathrm{x} / \mathrm{d}=1.0$ is far too large to be balanced by the negative area close to the summation axis. Changing the negative area has little effect on the summation as does changing the even closer positive area. Changing the area from $x / d=1.0$ to $x / d=\pi$ so that the 
forces balance does not balance moments as was previously shown. Further adjustment is required. Changing the area labeled (3) in Figure 9 requires changing (1) to maintain a force balance. Changing (3) enough to get $M=0$ requires lowering the stress level to $0.016 \mathrm{Fy}$ from $\mathrm{x} / \mathrm{d}=1.0$ to $\mathrm{x} / \mathrm{d}=\pi$. Then calculating the area and moment for the portion of the curve from $\mathrm{x} / \mathrm{d}=1.0$ to $\mathrm{x} / \mathrm{d}=\pi$, the amount of change to area (1) that is required for equlibrium can be solved for. With a known value for area (1), the maximum stress at the weld can be solved for. This calculation indicates the stress at the weld would be 2.758 times the yield stress when the ultimate stress. for the tube as determined by Tran (11) is 1.14 times the yield stress. A residual stress of 2.758 times yield at the weld is not reasonable or possible. Nor does the rapid decrease of residual stress at $\mathrm{x} / \mathrm{d}=1.0$ to a level of $0.016 \% / \sigma \mathrm{y}$ through the rest of the curve seem logical.

A more likely solution is to change area (3) to be larger positive in the section $\mathrm{x} / \mathrm{d}=1.0$ to $\mathrm{x} / \mathrm{d}=2.0$ and balance that with a smaller negative from $x / d=2.0$ to $x / d=\pi(F i g .10)$. This shape begins to look similar to Chen and Ross's (4) curve (see Figure 13) but the magnitudes are different. The only viable alternative is to assume the residual stress pattern is not symmetrical about the weld axis. There is no available evidence to support the assumption that the residual stress distribution 


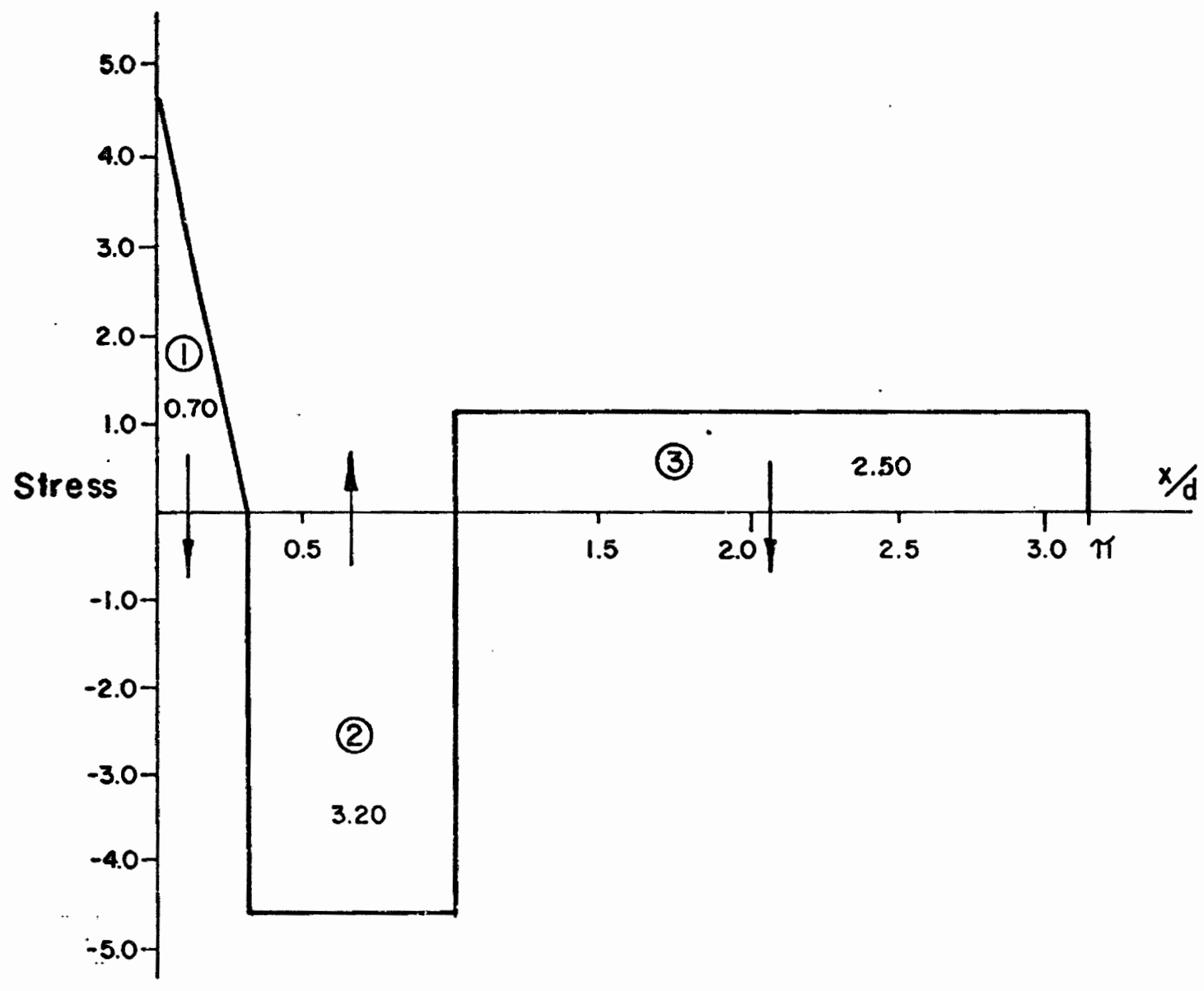

(1) $\frac{x}{d}=0.1 \quad \alpha=5.7295 \quad$ L.A. $=(1-\cos \alpha) r$

$$
=(1-\cos \alpha)(0.9035)=0.005
$$

(2) $\frac{x}{d}=0.3+0.35=0.65 \quad$ L.A. $=0.184$

$\begin{array}{lll}\text { (3) } & \frac{x}{d}=1.00+\frac{2.14}{2}=2.071 & \text { L.A. }=1.337\end{array}$ 


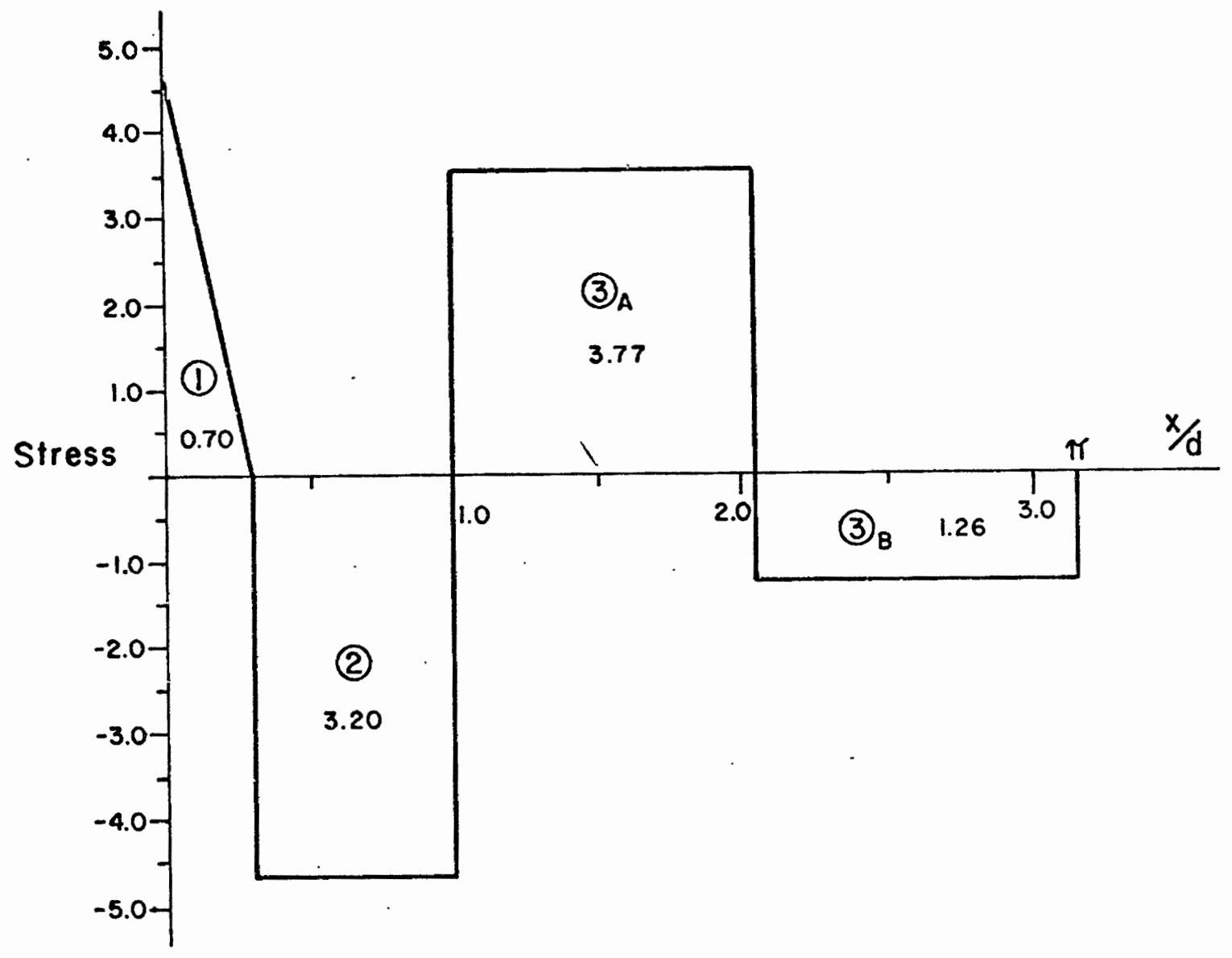

Figure 10. Approximate curve 
is not symmeticic. Tran took measurements from each half of the tube but he did not select mirror image points. Chen and Ross (4) also took values around the tube but did not separate the reported values in their representation of the data.

If the values for the portion of the curve from $\mathrm{x} / \mathrm{d}=0$ to $\mathrm{x} / \mathrm{d}=1.0$ are held constant and only the portion of the simplified curve from $x / d=1.0$ to $x / d=\pi$ is changed it is possible to show that for any. selected point of change from positive to negative, there is only one unique set of areas that will satisfy summation of forces and summation of moments equal to zero.

Summarizing then it is possible to use a simplified model of any residual stress curve to check for an approximate summation of forces and moments. Then using a more detailed description of the curve, e.g. 20 to 100 values around the tube scaled from a sketch of the curve, and using the previously described summation program, a more exact check for balance can be made. If the result is outside the required accuracy limit, the ordinates of the curve can be adjusted and the balance rechecked. By choosing unit values of change over one part of the curve, an indication of the effect of changing those ordinates on the total balance can be obtained. Using that information for further refinement makes the required iteration procedure converge on the required accuracy limit very quickly. 
Using this technique, it is possible to balance a possible curve shape in less than ten trial runs with the summation program. No attempt is made to find a mathematical expression describing these curves. 
CHAPTER IV

\section{RESIDUAL STRESS DISTRIBUTION CURVES}

Tran (11) has shown a curve that represents the data points obtained from his measurements by the hole drilling technique (Fig. 11). By dividing the curve into sixty-two segments and scaling the ordinate of the residual stress at each point and inputing these values into the summation program, an indication of the degree of imbalance was obtained. The summation of moments gave an imbalance of $10.9 \%$ My and the summation of forces an imbalance of $0.7 \%$ Py, both of which are certainly within experimental tolerance. The problem is that the M-P- $\varnothing$ Program requires a much higher degree of accuracy; $0.1 \%$ My and $0.005 \%$ Py. Therefore, some adjustment of the curve is required. Returning to a simplified model of this curve (Fig. 12) an approximate balance of forces and moments can be attempted. The areas labeled 1,2 and 3 can be idealized as forces concentrated at the center of gravity of the areas. Force 1 is positive and close to the crown, Force 2 is negative and within $x / d=1.0$ and Force 3 is positive at approximately $x / d=2.0$. Forces 2 and 3 are close to equal but of opposite sign. 


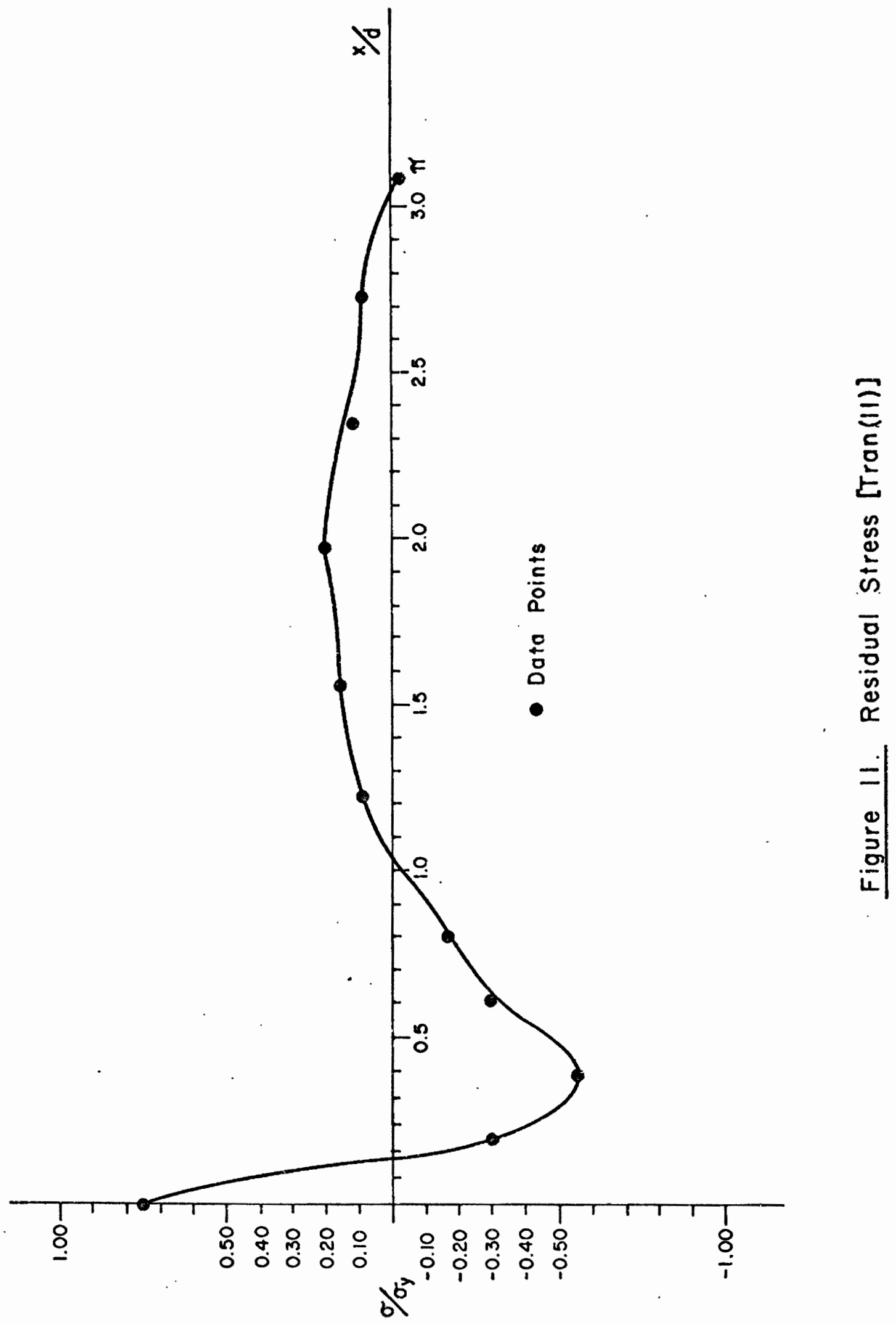




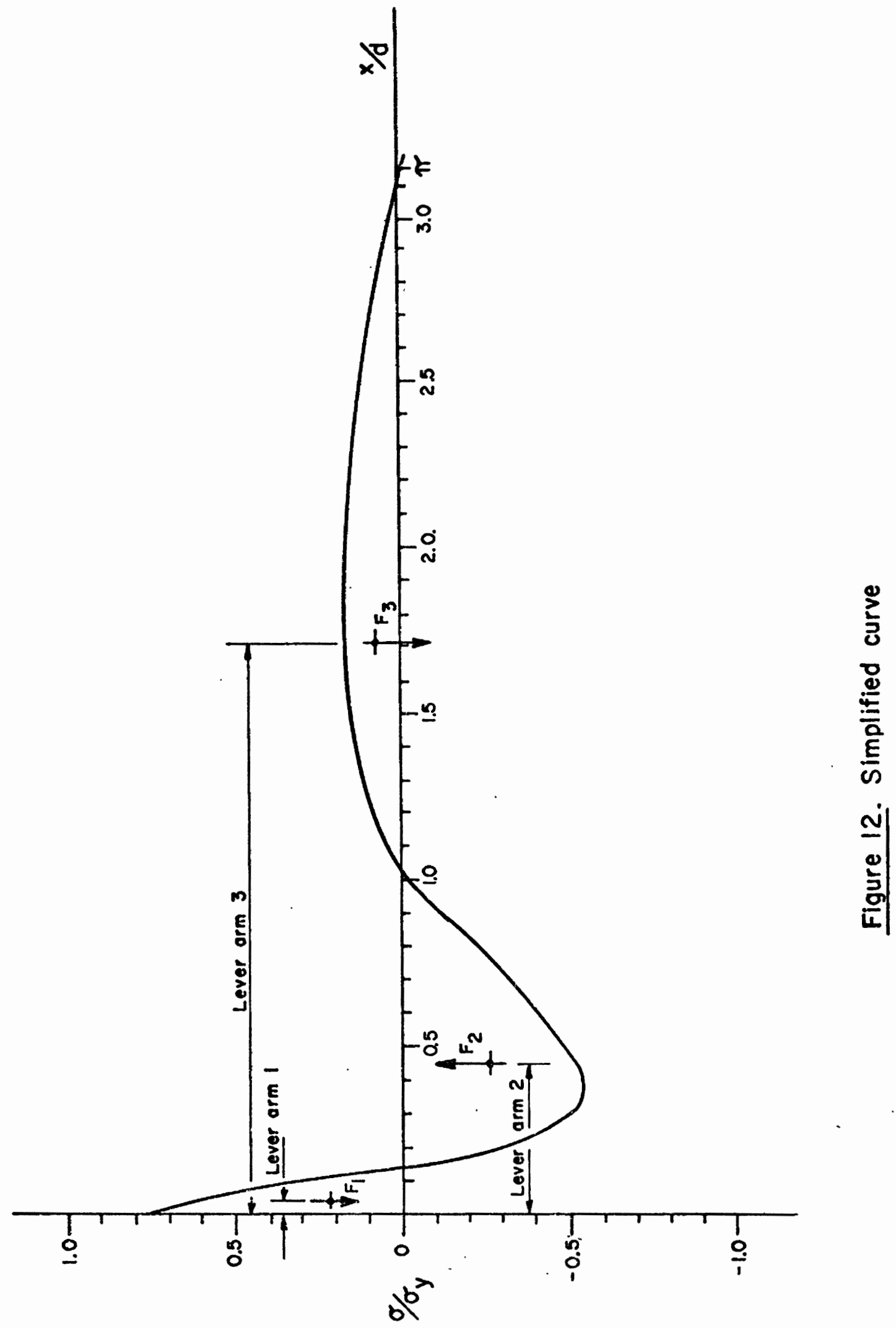




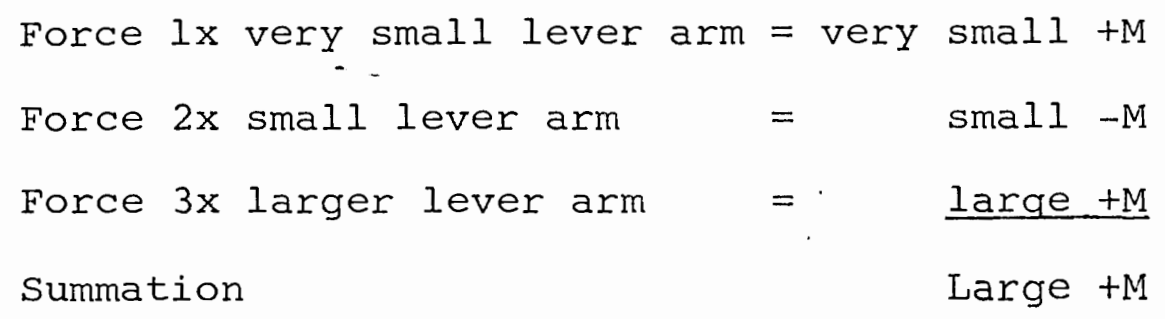

When Force 2 and 3 are nearly equal in magnitude, it is not possible to obtain static equilibrium. Therefore the curve Tran (11) suggests cannot be balanced without some major changes to the ordinates of the residual stress curve.

Chen and Ross (4) have shown a curve describing the residual stress distribution (Fig. 13) which they obtained from a combination of their measurements and theoretical considerations done by Marshall (7). The first portion of the curve from $\mathrm{x} / \mathrm{d}=0.0$ to $\mathrm{x} / \mathrm{d}=1.0$ is similar in shape to Tran's but of a different magnitude. This curve does not match all of their data points, but was selected based on close agreement. Dividing the curve into 62 segments as was done on the previous curve, scaling the ordinates and using the summation program gave results that could be compared with equilibrium and the results from Tran's curve (Fig. 13). The summation of moments gave an imbalance of $0.4 \% \mathrm{My}$ and a Force imbalance of $0.06 \%$ Py. This is again outside the limit required making further adjustment necessary. Selecting portions of the curve to change, it was discovered that. very slight changes in the latter $(x / d \geq 1.0)$ portion of the curve affected the total moment balance appreciably. The part of the curve 


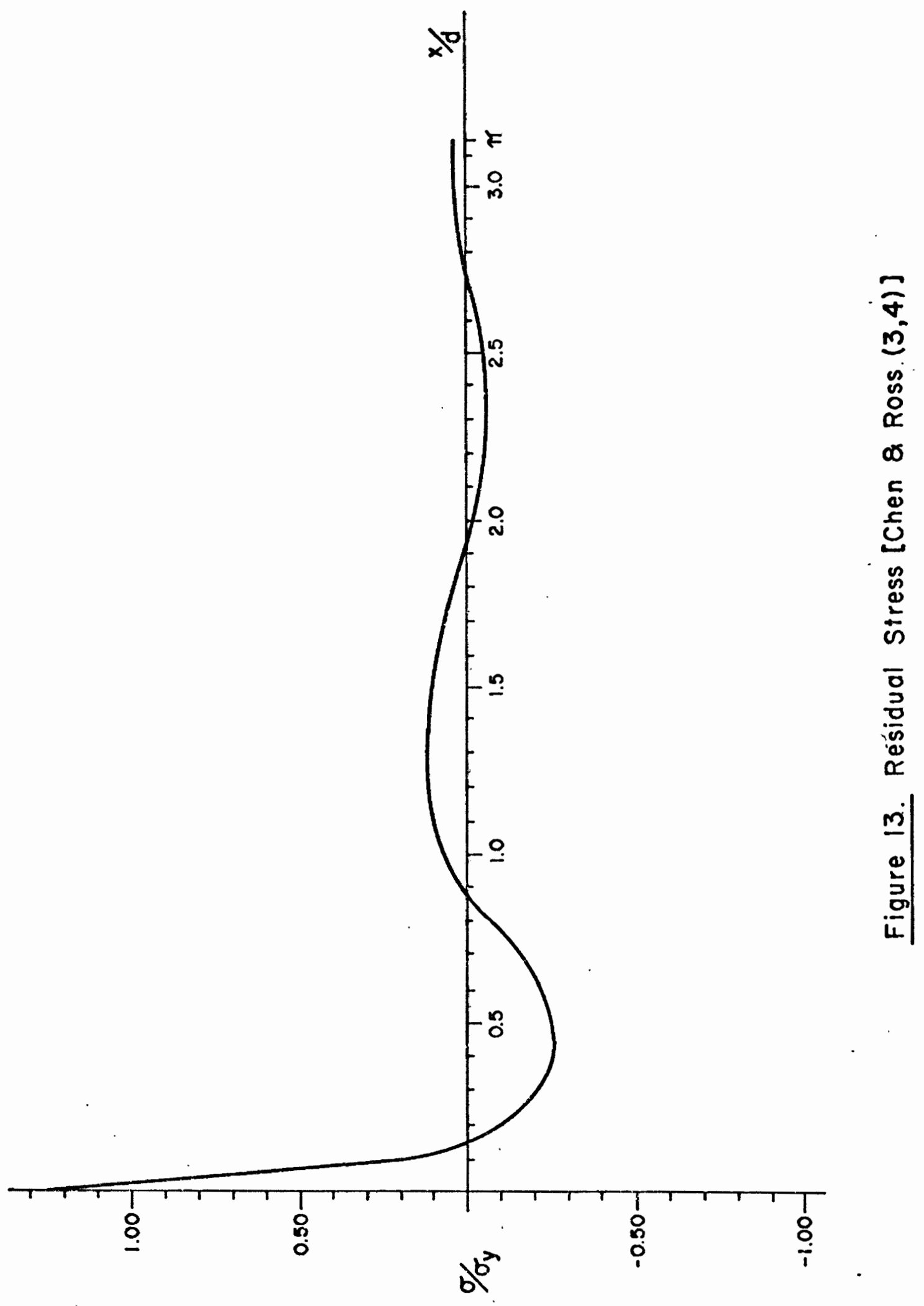


with the most difference between the actual data points and the suggested. curve were selected for change. After five iterations through the summation program, adequate approximation of equilibrium was obtained. Comparing the final curve with the starting curve (Fig. 14) it can be seen that the change required was small. Even though equilibrium is approximated with this curve, it does not consistently match the data presented by Chen and Ross (4) and $\operatorname{Tran}$ (1I) particularly over the portion from $\mathrm{x} / \mathrm{d}=1.0$ to $\mathrm{x} / \mathrm{d}=\pi$. Other possibilities needed to be explored. One alternative is to use an average between chen and Ross's curve and Tran's over the range $0 \leq x / d \leq 1.0$ with a positive and negative maximum between $1.0 \leq \mathrm{x} / \mathrm{d} \leq \pi$. As has been previously demonstrated, this is the minimum number of fluctuations that can render a possible solution. After balancing the curve to the required limit, it can be seen that the required curve areas imply larger positive and negative stresses over the latter portion than can be justified by the available data (Fig. 15).

Examining the curve shape in general, for changes in the first maximum of compressive stress, the behavior of the later portions of the curve can be predicted. Assuming that the curve crosses the axis at approximate multiples of $\mathrm{x} / \mathrm{d}=1.0$ and beginning with a curve like Chen and Ross's (Curve 1 Fig. 16) it is possible to show what changes will need to be made to maintain equilibrium 


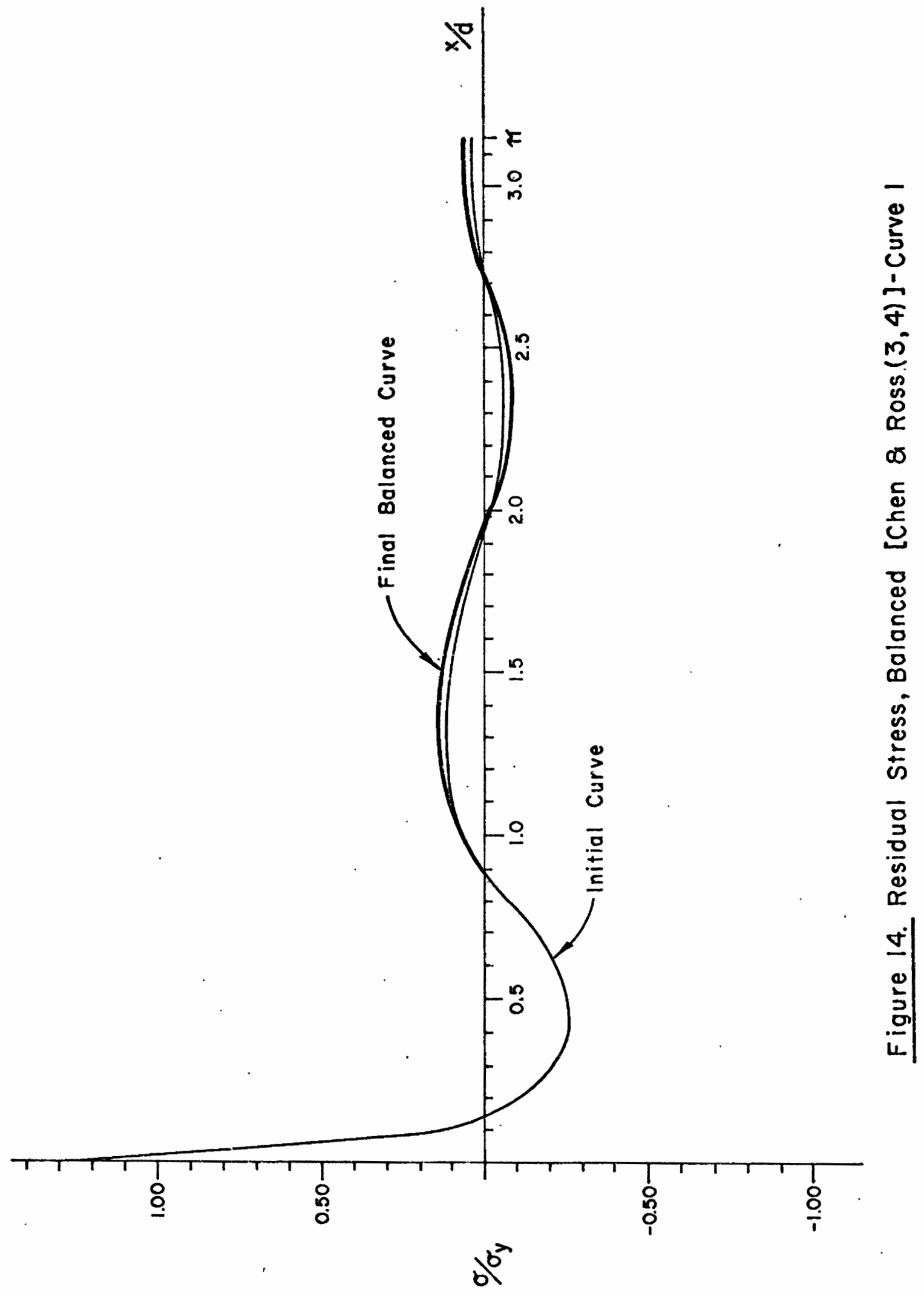



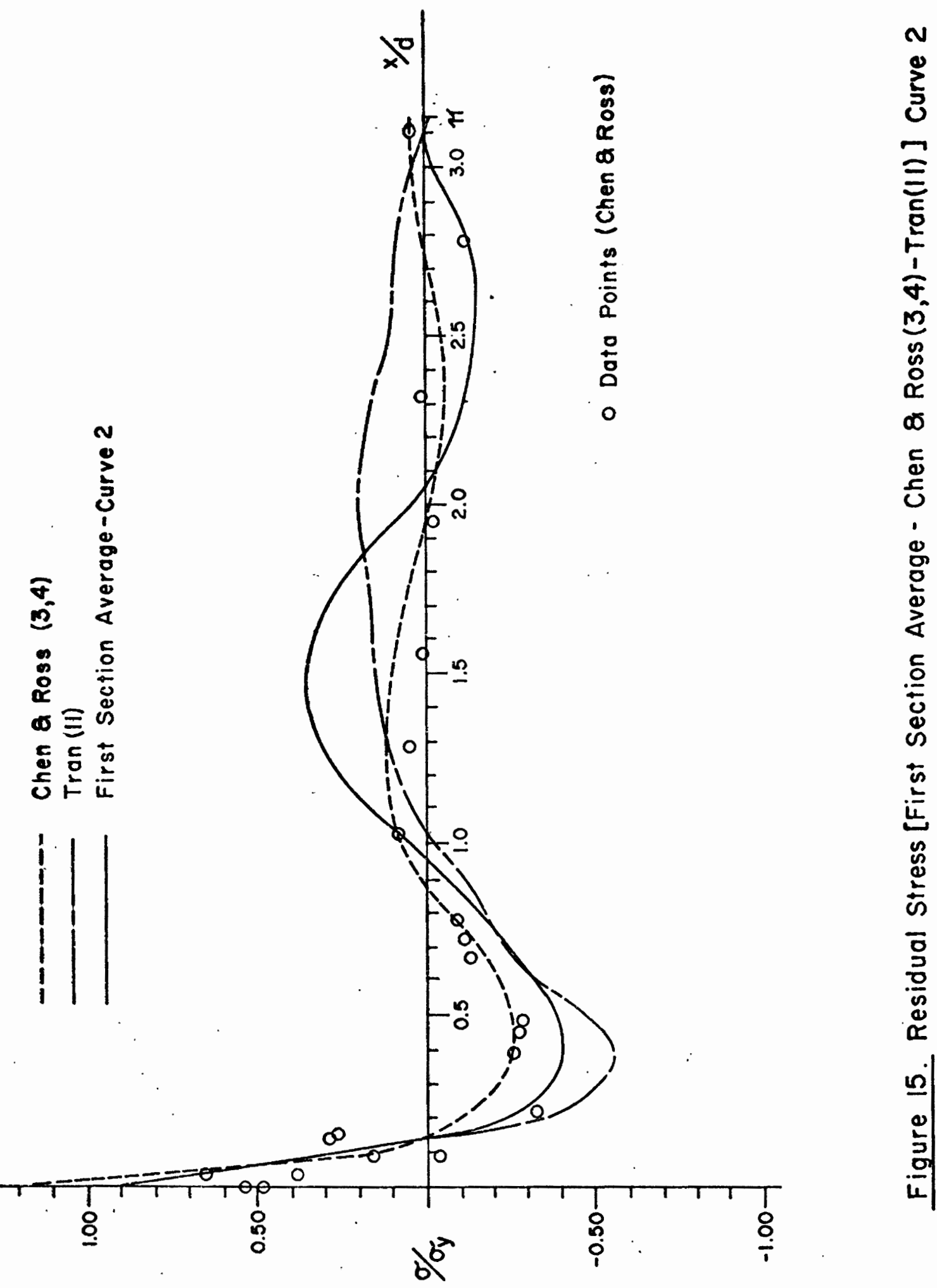

:

高

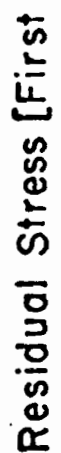

흔 


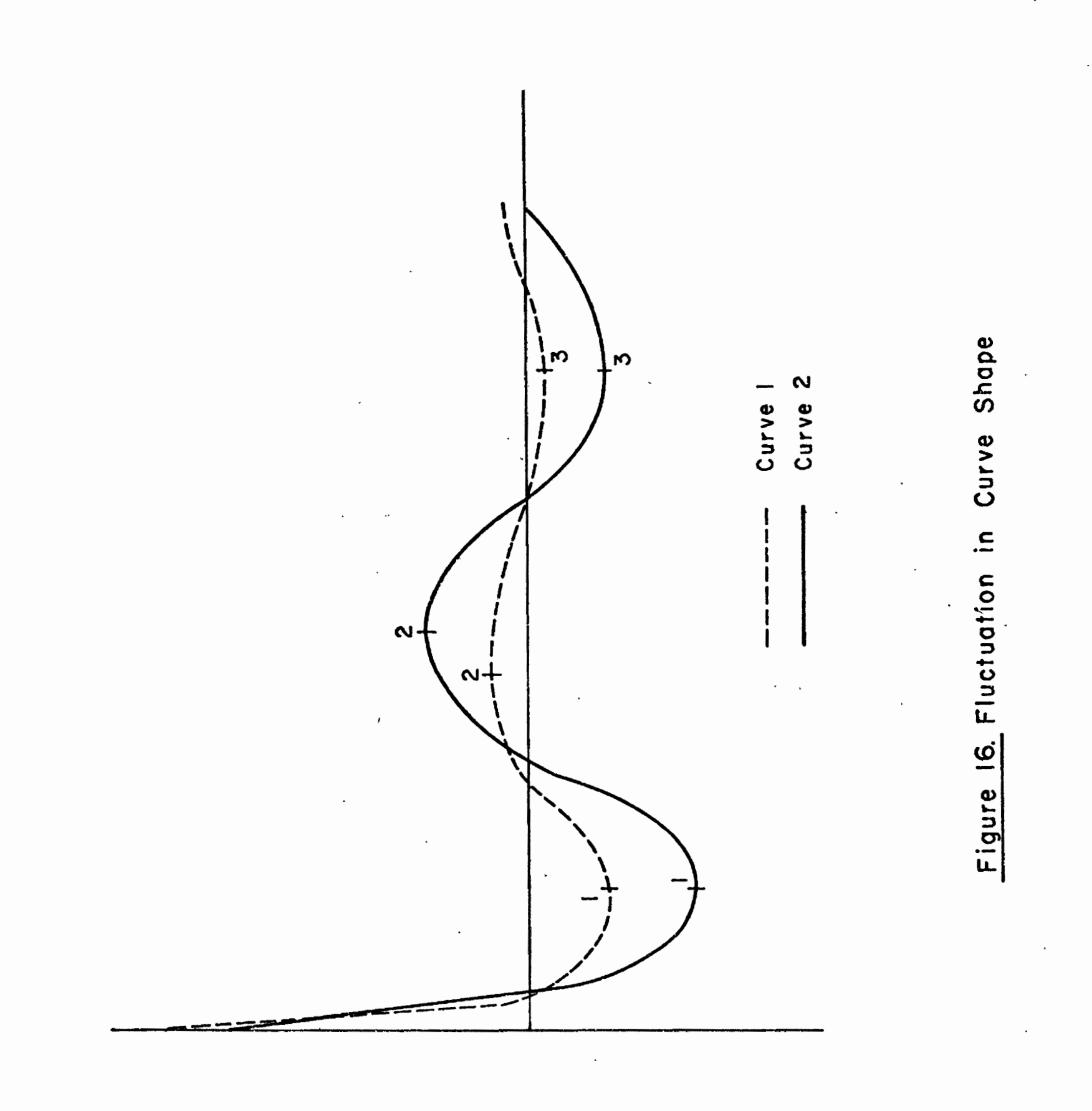


for a change in magnitude of the first negative maximum. Curve Number 1 is in equilibrium. If Curve 2 is generated by adjusting Point 1 to a different magnitude and it is assumed the curve still crosses the axis at multiples of $x / d=1.0$, the general shape for $x / d \geq 1.0$, assuming a smooth curve, is determined by consideration of moment and force equilibrium. Adjusting Point 1 to a larger negative magnitude means Point 2 must also increase in magnitude to balance the additional negative force from the change in Point 1. The moments are not in balance now since equal forces have been added but at different lever arms. Therefore point 3 must be adjusted to generate more negative moment to balance the positive moment from adjusting Point.2. Adding negative force from 3 means point 2 will have to increase which again results in a moment imbalance, although one of smaller magnitude. Continuing the iteration between the portion of the curve at 2 and 3 will result in a unique solution with larger final magnitudes for Points 2 and 3. Therefore the portion of the curve from $x / d=1.0$ to $\mathrm{x} / \mathrm{d}=\pi$ is very sensitive to changes in magnitude of Point 1 .

An example of this sensitivity is the consideration of a curve generated by smoothing out Tran's curve from $\mathrm{x} / \mathrm{d}=0.0$ to $\mathrm{x} / \mathrm{d}=1.0$ (Fig. 17). After balancing the curve as previously described, the ordinate of the positive maximum was three times the yield stress which is consider- 


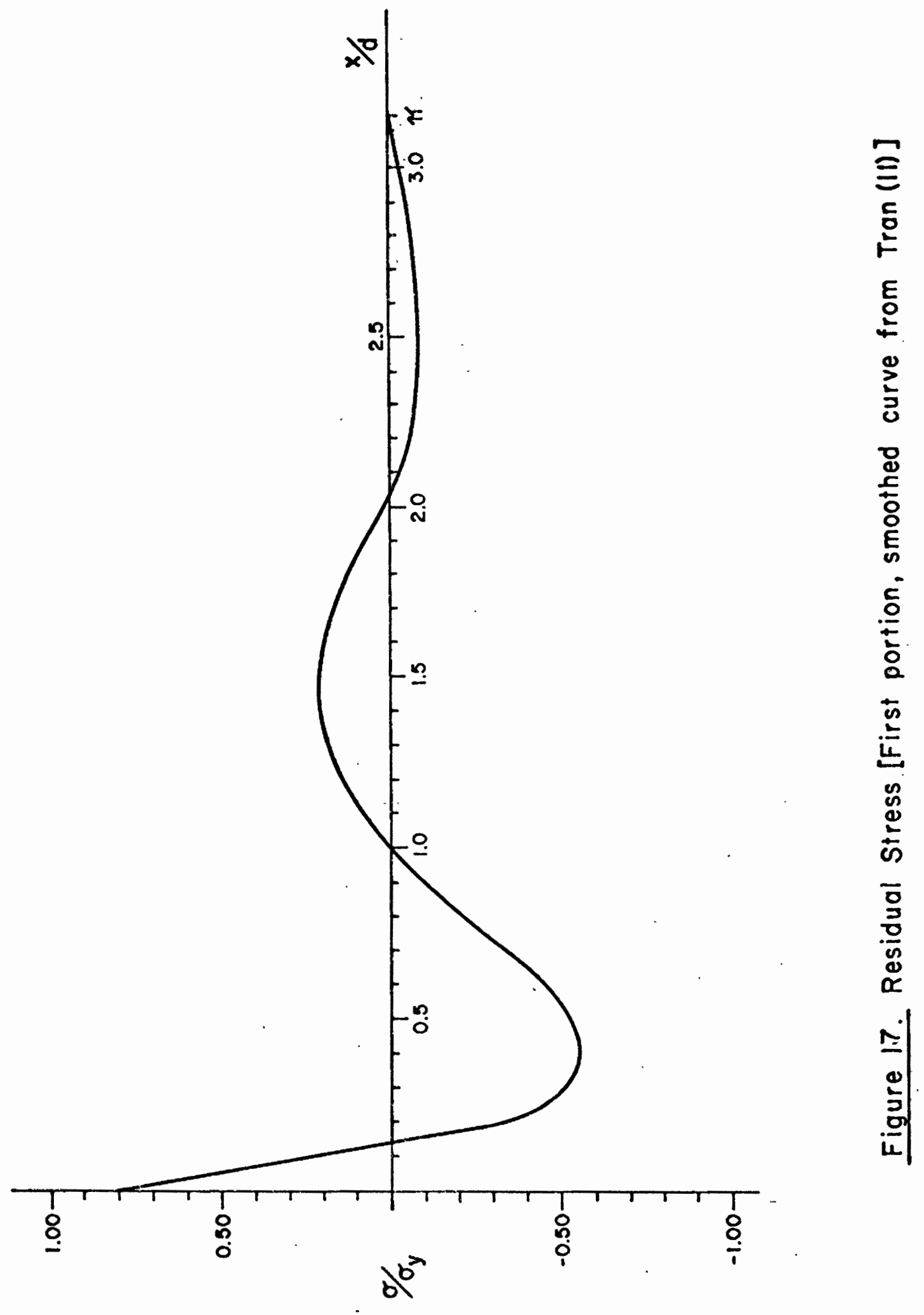


ably greater than the stress at the weld. This means the single data point Tran found at approximately $\mathrm{x} / \mathrm{d}=0.8$ is inaccurate and should not be considerated in generating a curve, since the condition of equilibrium demands unrealistic stresses using the point.

In general, a smooth curve shape with a maximum tensile stress at $\mathrm{x} / \mathrm{d}=0$, a maximum compressive stress at $x / d<1.0$, and zero stress at approximately $x / d=1.0$, $\mathrm{x} / \mathrm{d}=2.0$ and $\mathrm{x} / \mathrm{d}=3.0 \mathrm{will}$ exhibit an easily determined behavior for changes in the magnitude of the first compressive maximum. It has been shown herein that using a base curve of this shape, small changes in the compressive stress maximum at $\mathrm{x} / \mathrm{d}<1.0$ require larger changes in the remaining tensile and compressive stress regions. It is, therefore, possible to modify the effect of a reported data point on the smooth curve by considering the change required in the rest of the curve. A data point which causes, by its inclusion in a smoothed curve shape, an unreasonable stress distribution for the other parts of the curve can be identified using this method.

Several other curves were generated in an attempt to use the combined data from Chen and Ross $(3,4)$ and Tran (11). The most consistant results were obtained from a curve using the first part of Chen and Ross's curve from $\mathrm{x} / \mathrm{d}=0.0$ to $\mathrm{x} / \mathrm{d}=1.0$ and forcing the latter portion of the curve to one positive maximum and one negative maximum with the curve approaching zero at $x / d=\pi($ Fig. 18). 


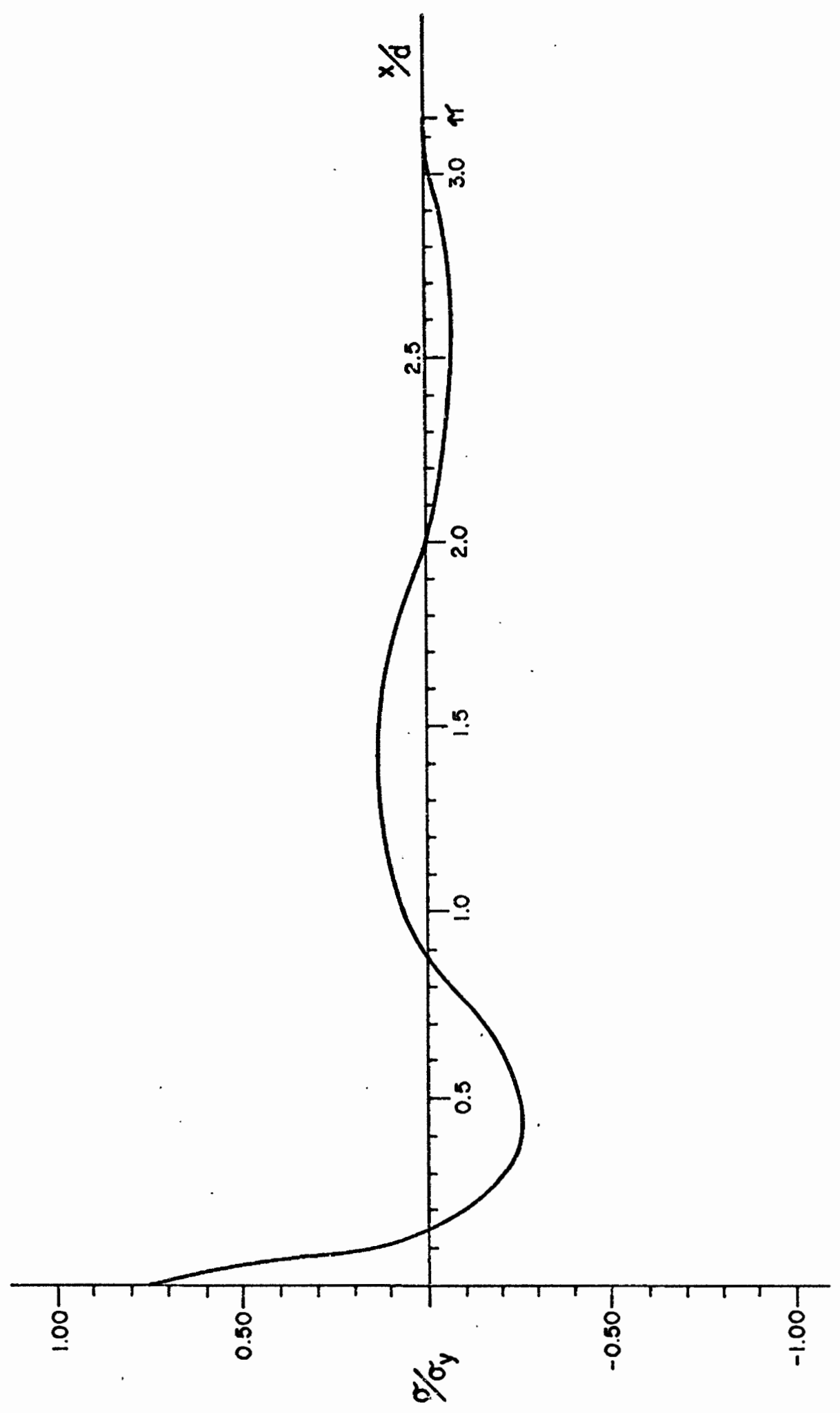

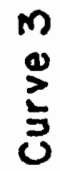

ह

$\stackrel{0}{3}$

오

용

능

7

m

:

$\infty$

它

吕

cs

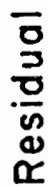

음

ํㅣㄴ 
Three curves of the several curves developed were selected to generate M-P- $\varnothing$ curves with: a balanced version of Chen and Ross's curve (Curve 1, Fig. 14), section from $\mathrm{x} / \mathrm{d}=0.0$ to $\mathrm{x} / \mathrm{d}=1.0$ average between Tran and Chen and Ross's (Curve 2, Fig. 15), and Chen and Ross's curve forced to two maximums from $x / d=1.0$ to $x / d=\pi$ (Curve 3 , Fig. 18) . 


\section{M-P-Ø CURVE GENERATION}

Once the description of the residual stress distribution was complete, Wagner's (13) program was used to generate $M-P-\varnothing$ curves. It is important to understand the method that Wagner's program uses to calculate these curves. There are four major stages: assigning the appropriate value of residual stress and strain to each element, application of a percentage of the stub column yield load to each element, assigning a curvature to the cross section, and calculating the moment corresponding to a state of equilibrium. For one value of axial load at one curvature, the moment is then calculated. By repeating these steps for several axial loads at different curvatures, it is possible to generate a family of $\mathrm{M}-\mathrm{P}-\varnothing$ curves. Several intermmediate steps are involved in each of the four major stages (Fig. 19).

In the first stage of the program all of the required data is read; number of layers, number of elements, diameter of tube, wall thickness, modulus of elasticity, yield stress, the number of axial load values, and the number of curvature values. Then the values of axial load and curvature are read. The program has the option of using 

Assign appropriate residual stress and $\operatorname{strain}\left(\varepsilon_{r}\right)$ value
to each element.

Apply axial load (P) and

STAGE 2

calculate the strain $\left(\varepsilon_{\mathrm{a}}=\mathrm{P} / \mathrm{AE}\right)$

Calculate the total strain $\left(\varepsilon_{t}=\varepsilon_{r}+\epsilon_{a}\right)$ for each element

Using $\varepsilon_{t}$ and the stress-strain relationship find the

total Eorce on the cross section ( $F)$.

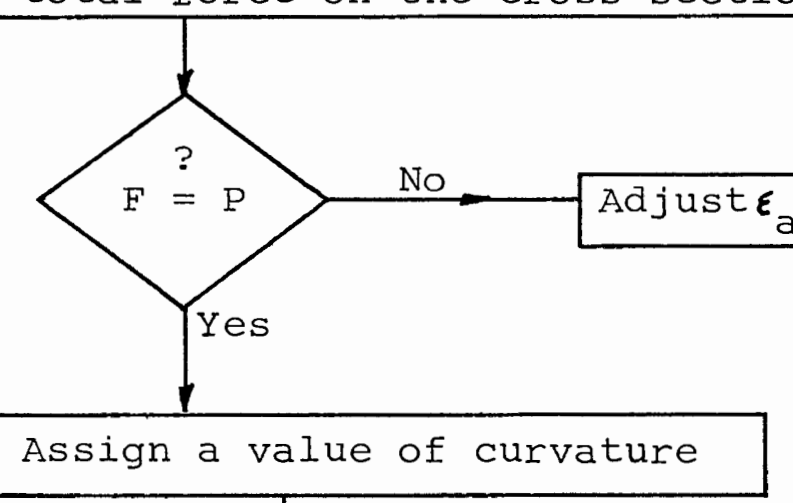

STAGE 3

Determine the strain on each element

due to curvature $\left(\varepsilon_{\phi}\right)$

Calculate the total strain for each element $\left(\boldsymbol{\varepsilon}_{t}=\boldsymbol{\varepsilon}_{r}+\boldsymbol{\varepsilon}_{\mathrm{a}}+\boldsymbol{\varepsilon}_{\boldsymbol{\phi}}\right)$ STAGE 4

Using $\varepsilon_{t}$ and the stress-strain relationship; determine the total force $(F)$ and the bending moment (M) on the cross section

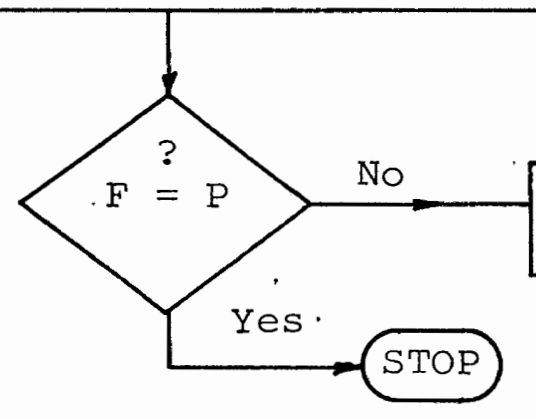

Fiqure 19 Flow diagram for calculation of M-P- $\varnothing$ data 
a tabulated stress-strain curve or a bilinear stress-strain curve and this information is entered next. The residual stress and strain. is read in and the applicable value assigned to each element. For each layer the average radius, arc length of the elements and the area of the elements is calculated. From the results of Tran's work it is apparent that consideration of layers in the tube wall is not required. for the description of the residual stress-strain distribution since these do not vary through the thickness of the wall. The strain, curvature, bending moment and axial load at first yield are calculated next. Then the distance from each element to the centroid of the cross section, the total cross sectional area, plastic modulus, flexural stiffness, plastic hinge moment and shape factor are determined. The description of the problem is now complete.

The next stage is to apply the first axial load (expressed as a percentage of Py) to the cross section and calculate the axial strain. An iteration loop is performed next to determine the correct value of axial strain. This is required since it is possible for the summation of the residual strain and the axial strain on any particular element to exceed the yield value. In these cases the summation of the elemental stress available to resist the axial load is less than predicted by elastic theory. The residual stress distribution is an initial condition and 
cannot be changed, therefore the additional force must be supplied by the elements that have not yet reached yield. The stress distribution and magnitude is determined by increasing the strain on all elements by the same amount and then calculating the resulting stress using the material stress-strain information (tabulated input of any stressstrain curve or a bilinear curve as previously described). The summation of available elemental stress at each increment of strain is compared to the total applied force and when they are approximately equal the interation is stopped and the values for each element are stored for further use.

The next stage involves assigning a value of curvature to the cross section. The neutral axis is assumed initially to be at the centroid of the cross section and the corresponding strain at each element is calculated. The summation of strain due to residual stress, axial load and the imposed curvature is limited to the strain at first yield as previously described for residual stress plus axial load for each element. The resulting strains are used with the material stress-strain information to determine the resulting stress for each element. These stresses are summed and the resulting total thrust is compared with the applied axial load. If the axial load and thrust are not equal the location of the neutral axis is shifted and the strains recalculated. The iteration is continued until approximate equality is reached.

The last stage is to calculate the summation of moments for the final stress-strain state. The result is a 
value of moment at a particular curvature for one value of axial load. This gives one point for determining one $M-P-\varnothing$ curve. The process for finding the moment is repeated for each curvature at one value of axial load. That gives all the points of the M-P- $\varnothing$ curve for that particular axial load. The program then selects the next value of axial load and begins at stage two repeating the process of solving for moment at each value of curvature until all values of axial load input have been used. The values of moment, axial load and curvature are then output in tabular form. This information can then be used in the failure load program as described by wagner (13).

In order to completely study the effect of the residual stress distribution on failure loads it is necessary to determine the effect orientation of the axis of bending with respect to the axis of the weid has on the $M-P-\varnothing$ curve. The concern is to find the weakest axis of bending to be sure that the controlling case has been found. The axis of bending selected by wagner $(12,13)$ passed through the weld (Fig. 20) for which results for the M-P- $\varnothing$ curves were compatible with theory $(1,2,5,8)$. Considering the axis orientation with the weld at the top of the pipe and the bending axis through the horizontal diameter as the zero or reference point, any orientation can be described as the number of degrees of rotation of the bending axis from the reference position. Thus the orientation wagner 


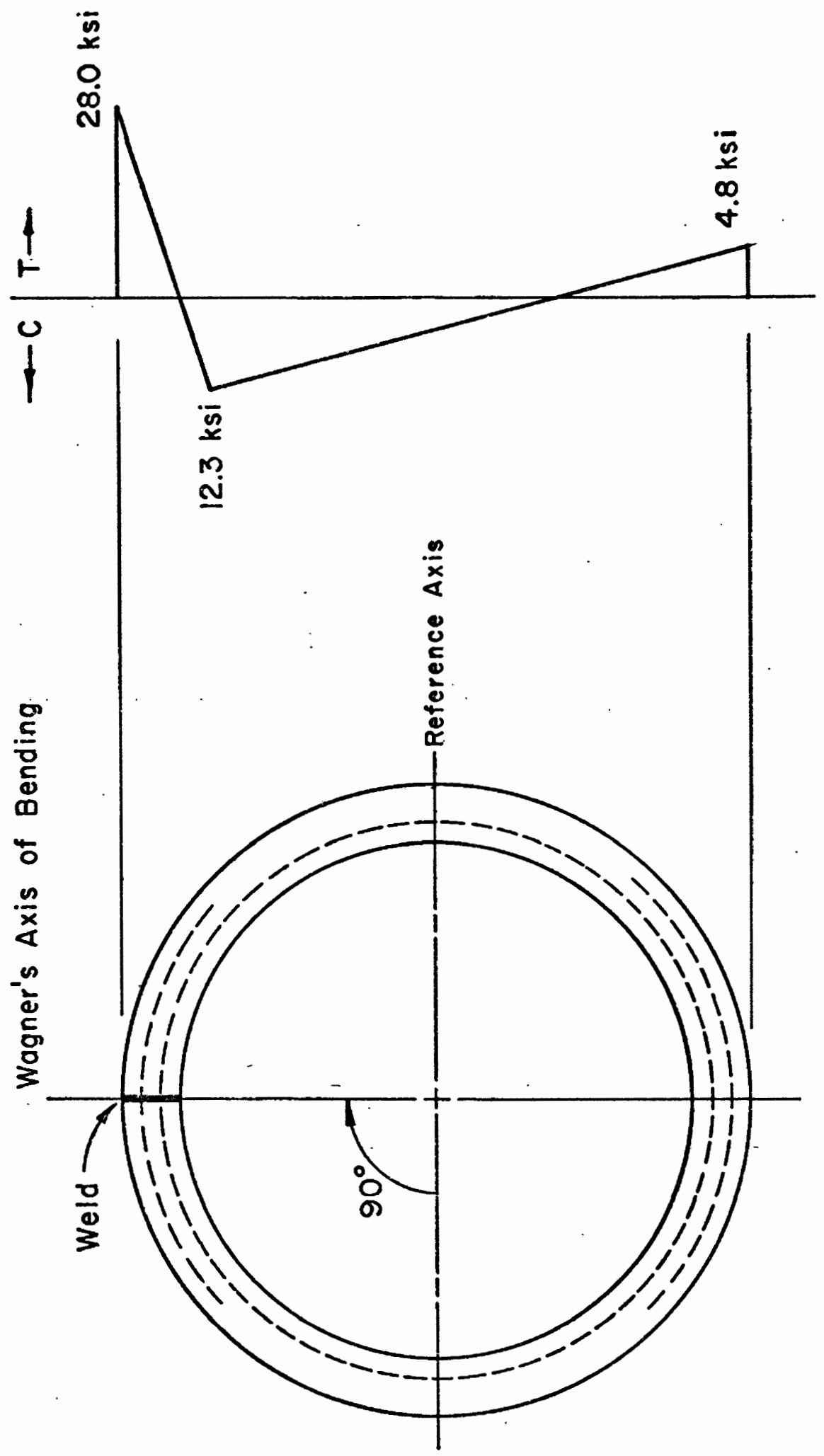

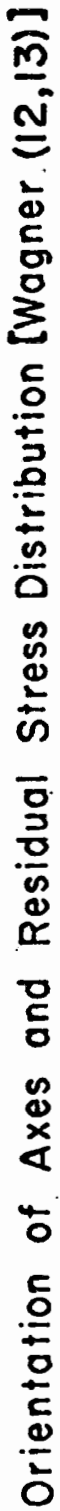

\begin{tabular}{c}
0 \\
N \\
0 \\
$\frac{1}{3}$ \\
0 \\
\hline 1
\end{tabular} 
$(12,13)$ selected would be at $90^{\circ}$ from the reference. When any other orientation was selected, the results were not consistant with theoretical considerations $(1,2,5,8)$. The moments were not approaching the value for full plastic moment at large values of curvature. (Figs. 21,22, 23). Clearly there was a significant problem with either the proposed residual stress distribution or with the program. The first step was to verify the results obtained by Wagner for the distribution used with the orientation of $90^{\circ}$ from the reference. Comparing the results for this orientation of bènding axis showed exact agreement with the work done by wagner $(12,13)$.

Inputing zero residual stress and checking several axis orientations also showed exact agreement with the theoretically predicted behavior of the M-P- $\varnothing$ curves.

In order to pinpoint the problem Wagner's distribution (see Figure 20) was used. The axis of bending was assumed at the reference position and a summation of moments accomplished for the cross section at the end of each iteration to determine the correct axial strain. (This is described in the second stage of the program, see Fig. 19). Residual stresses were output to verify that there was no change occurring. (They are input constants and should not change.) The total stresses and strains were also output to verify that they did not exceed yield during the iteration procedures. All of the output verified the 


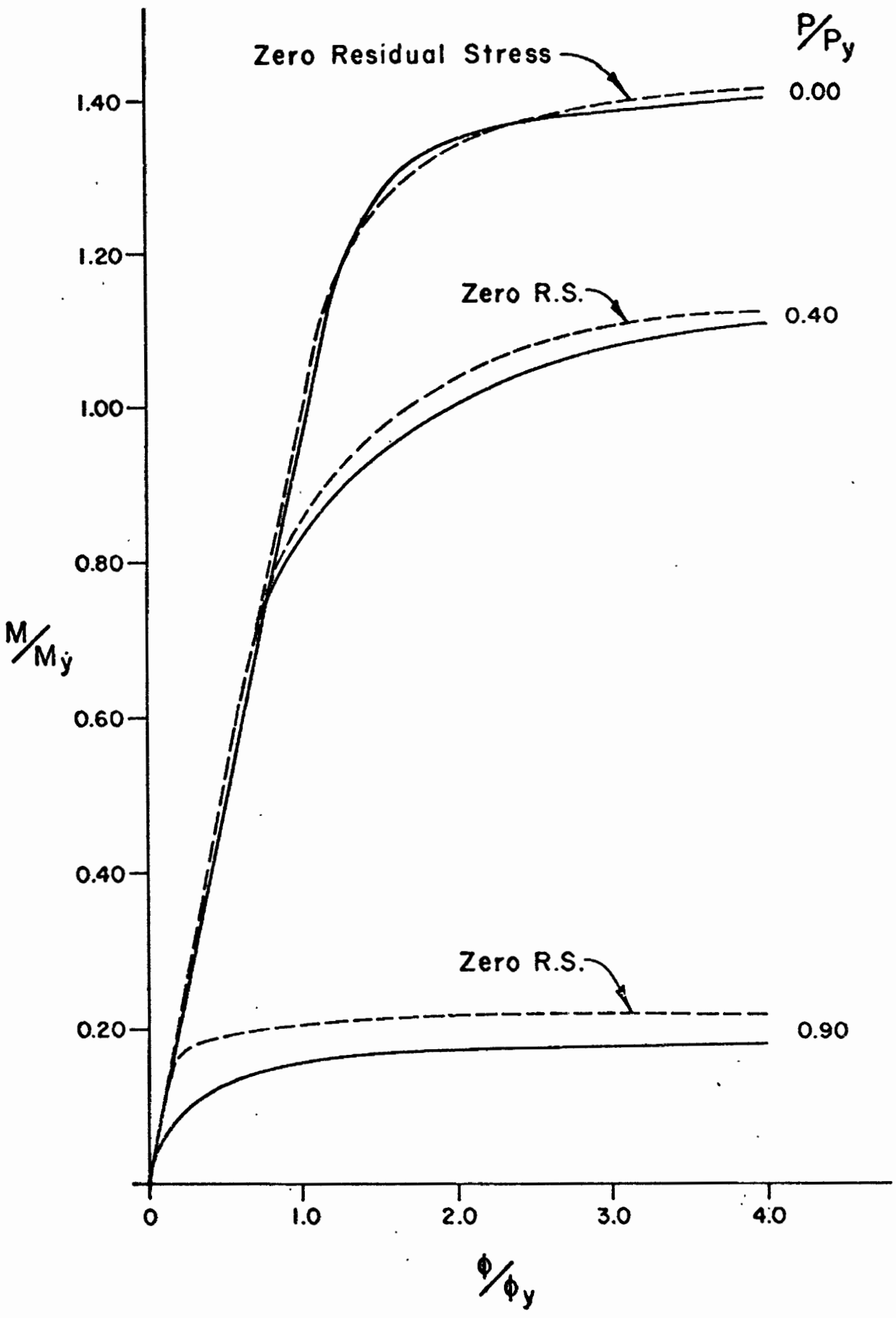

Figure 21. M-P- $\$$ Curve I(Chen \& Ross) 


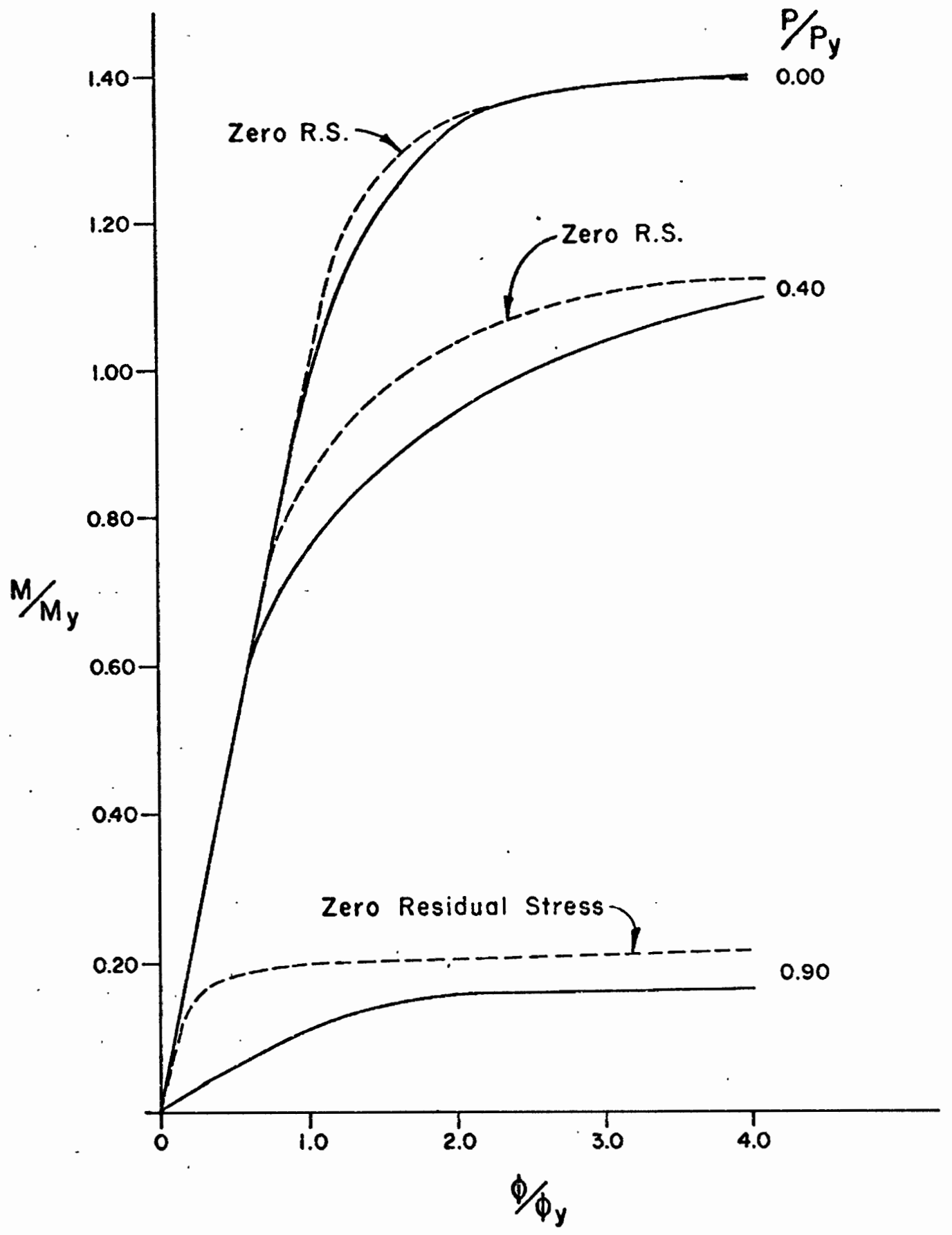

Figure 22. M-P- $\Phi$ Curve 2 (First Section Average) 


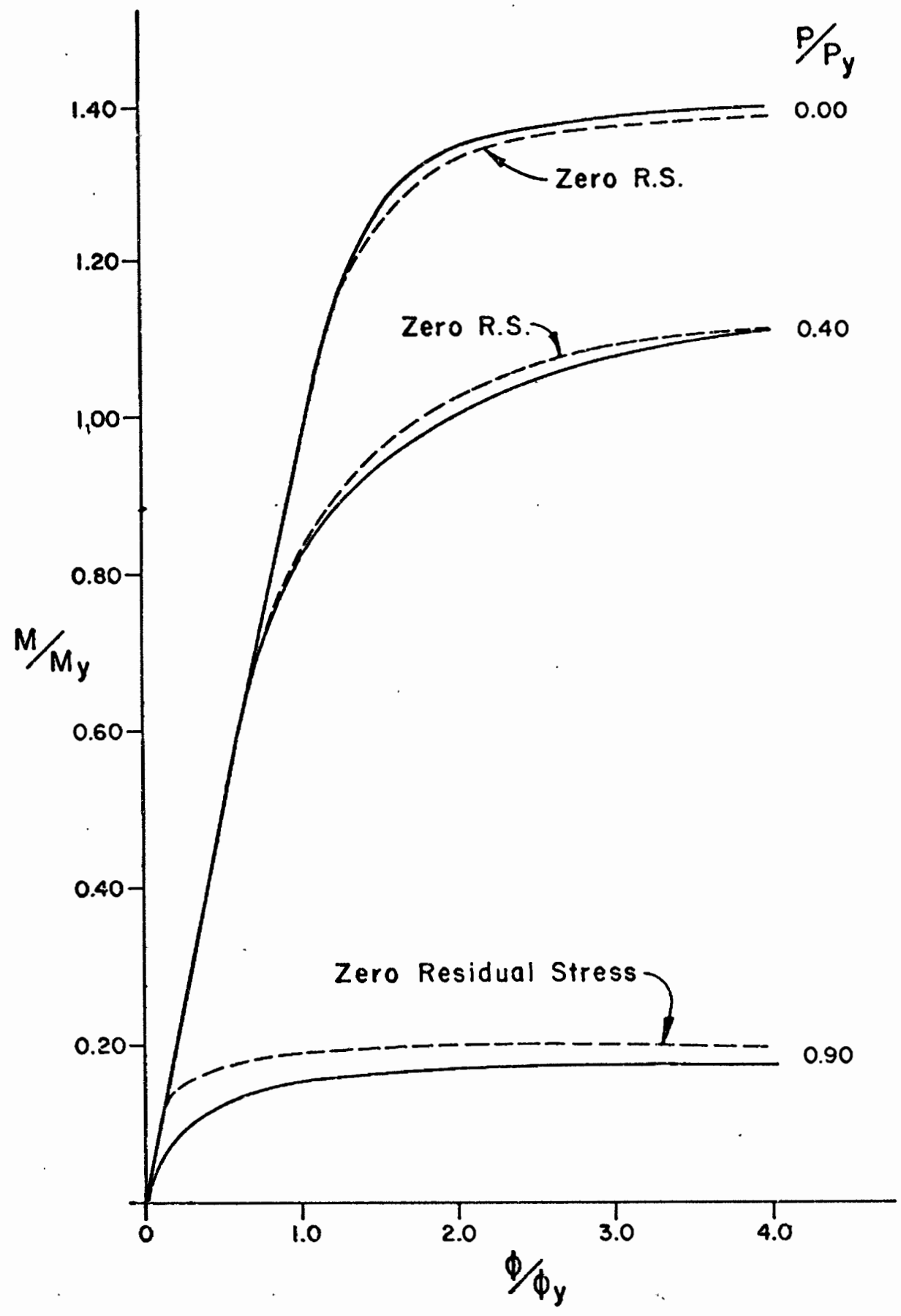

Figure 23. $M-P-\phi$ Curve 3 (Chen $\&$ Ross forced to two maximums) 
procedure with the exception of the summation of moments after finding the "correct" axial strain and corresponding stress. The summation of moments at this point should be equal to zero since the residual stress distribution is in equilibrium to begin with and only axial load has been added. Because the program does not alter the residual strain or the axial strain after this point in the program any imbalance produced here will be unchanged at the end of the iterations. The strains due to curvature are added after this and the total limited to the described material properties. The strain due to curvature at this value of axial load is calculated by subtracting strain. This strain due to curvature is used to find the stress on each element. Summing moments for these values the moment for the entire cross section is obtained. The thrust is calculated and compared to the applied force and they are not equal. The neutral axis is shifted and the moment and thrust are recalculated. This procedure in no way affects the values of strain that were solved for due to axial load. Therefore, if the cross section is not in static equilibrium after the solution for strains due to axial load, it will still not be in equilibrium after the "correct" location of the neutral axis has been found and the corresponding moment calculated.

The result of the summation of moments after completing the iteration to find the "correct" axial strain was not equal to zero in any case tested except for those with no 
residual stress and those with $90^{\circ}$ orientation of axis of bending with respect to the reference. The disparity between the theoretical plastic moment and the calculated moment for the $\mathrm{P} / \mathrm{Py}$ ratio was compared to the moment imbalance created during the solution for axial strain and found to be equal. In other words the sum of the calculated moment at some large curvature plus the moment created in the solution for axial strain equals the theoretically predicted plastic moment. It would seem the simplest solution would be to compute the moment due to curvature by a summation using the total stress instead of only that part of the stress due to curvature. That way the moment imbalance is incorporated in the final moment. The problem with this solution is that while the $M-P-\varnothing$ curve has the . correct starting point and is correct for large values of curvature, the midale portion has its shape changed considerably by the moment imbalance. The solution, then is to eliminate the moment imbalance created before entering the iteration loop to find the moment due to curvature. In this manner a residual stress distribution that is in static equilibrium will have an axial stress distribution added to it and the final result will also be in static equilibrium. This model is then showing the same behavior as the actual physical cross section.

To eliminate the moment imbalance, the first step was to determine how the imbalance is created. When the 
axial strain is being solved for the first step is to apply a uniform strain to the cross section computed from the average axial stress, $\mathrm{P} / \mathrm{A}$, and the material properties of the cross section. The axial strain is added to the residual strain for each element and the stress from this sum is limited by the described material properties (either tabulated or bilinear stress strain curve). If the sum of axial strain and residual strain is larger than the strain at first yield the available stress will be less than the average axial stress. That means the summation of thrust on the cross section will be less than the applied axial load. Therefore the strain over all the elements is incremented and the summation recalculated. This procedure continues until approximate agreement is reached between the calculated thrust and the axial load. If some of the elements above the center of gravity of the cross section are very close to yield from residual strain alone, the increase in strain for axial load will put them over yield. The stress available for axial load from them is less than the average, therefore the strain is adjusted. This increase in strain does not cause much if any increase in stress available from the elements that were at or over yield in the first iteration. The other elements now have an increase in stress over the average $\mathrm{P} / \mathrm{A}$. As the iteration process continues the distribution of axial stress may become even more irregular as other elements yield. When 
the iteration is complete the stress distribution is no longer uniform and the center of gravity of the resulting thrust is no longer located at the center of gravity of the cross section. Therefore a moment has been created during the iteration due to the yielding of some elements. (Fig. 24)

To explain why there was no imbalance using this program for the axis of bending taken through the weld ( $90^{\circ}$ to the reference) the residual stress distribution used must be examined. The distribution used was assumed to be symmetrical about the axis of the weld. Therefore the change in stress due to axial load on each element is also symmetrical about the axis of the weld. Since the change in stress is symmetrical about this axis, the resulting thrust is positioned somewhere along the axis. Therefore summing moments about this axis yields zero even though summation of moments about any other axis would not be. The cross section is not in equilibrium but the moment is only being taken about one axis in this program so the imbalance goes undetected for this one special case (axis of bending through the weld, $90^{\circ}$ with respect to reference chosen.) The $\mathrm{M}-\mathrm{P}-\varnothing$ curves generated for this case are then unaffected by the imbalance but for all other cases they would be. The case with the largest imbalance should be with the axis of bending at the reference position (perpendicular to the weld axis) and this was confirmed by a, test run of the program as written. 


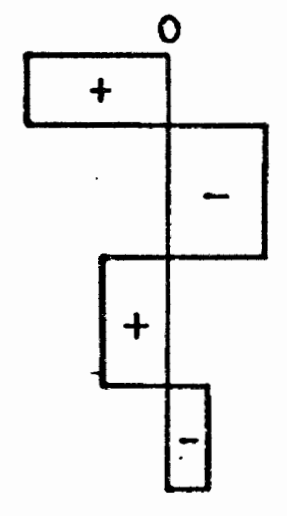

Residual Stress
0

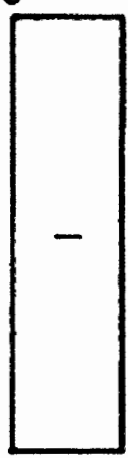

Axial Stress

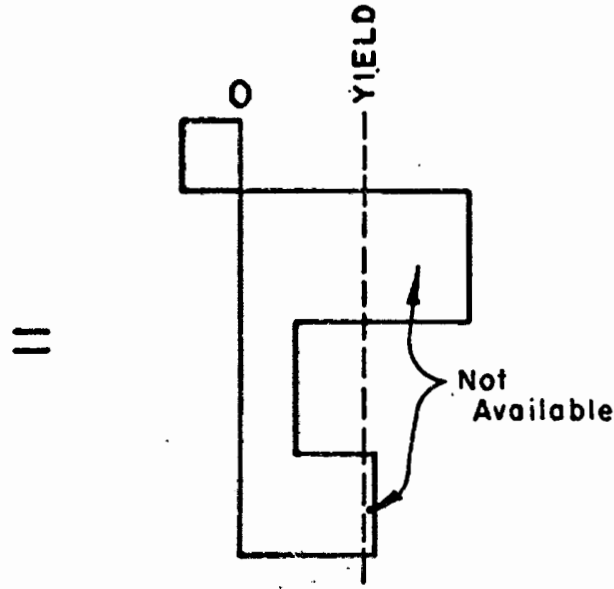

Summation (Not in

Force Equilibrium)

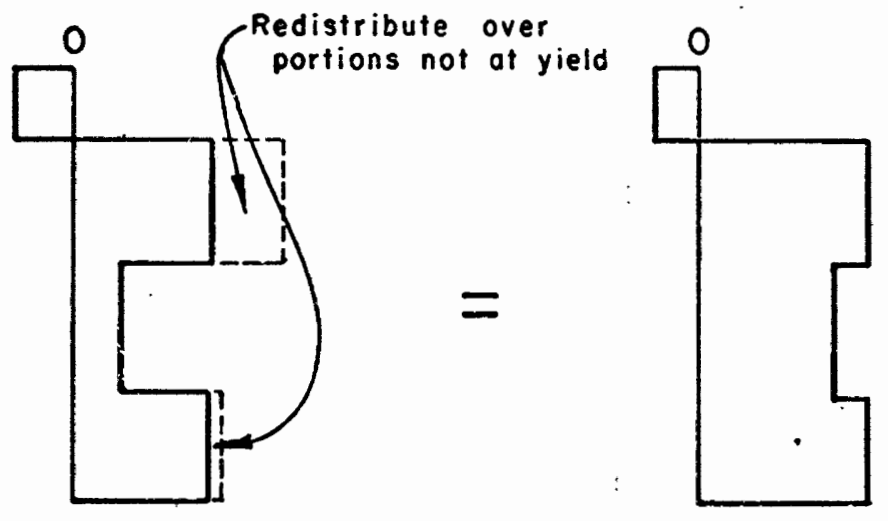

Summution

Summation (In Force Equilibrium)
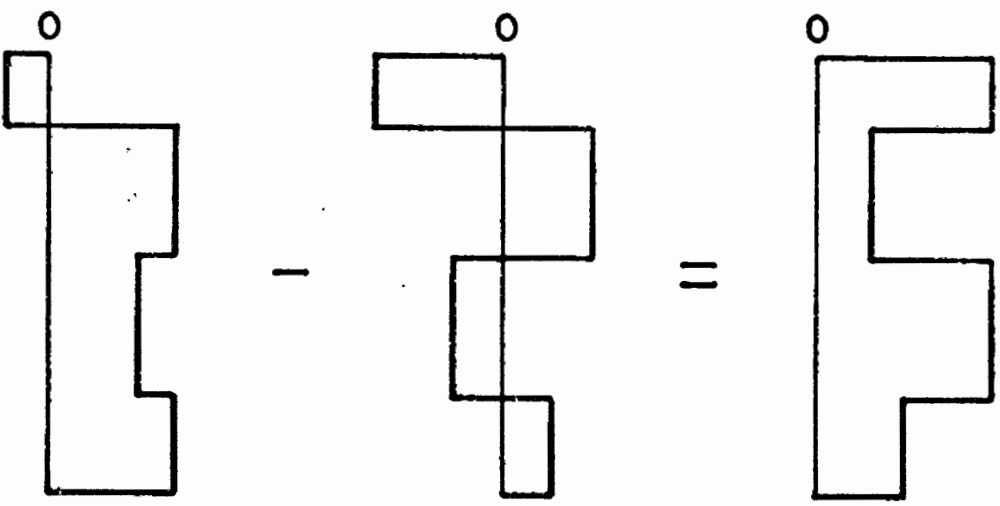

Not in Moment

Equilibrium

Figure 24. Moment Imbalance 
The problem was identified and several possibilities for a solution were explored. The method selected was to add another iteration after determining the axial strain to eliminate the imbalance created. The value of curvature that would generate a moment equal to the unbalanced moment was calculated assuming the section to be elastic. The sign of this curvature was reversed thereby changing the sign of the moment. Strains were calculated based on this curvature and added to the strain determined for axial load plus residual stress. This in effect would cancel the unbalanced moment leaving the cross section in equilibrium. The curvature used is actually the reverse of the curvature generated by the uneven axial stress distribution, which means the section ends up with curvature equal to zero. The procedure actually assumes a curvature (elastic) and iterates to find the correct curvature (produced by uneven axial stress). The iteration also must still limit the stress to that determined from tabulated or bilinear stress-strain curves. The iteration also involves checking the summation of thrust on the elements against the applied axial load for approximate agreement. The revisions required to the program were extensive and are shown in Appendix. II. Each axis rotation requires a separate run of the program.

Because various orientations of the bending axis were desired, the program was also modified to automatically 
rotate the bending axis to any whole element increment (for example: $360^{\circ} / 80$ elements $=4.5^{\circ} /$ element $45^{\circ} / 4.5^{\circ}$ / element $=10$ element rotation). Since a large number of M-P- $\varnothing$ curves were going to be generated, a graphics plot program was written to automatically plot each set of curves on a Cal-Com plotter. The plots for Wagner's residual stress distribution and the suggested curve 3 are shown in Figure 25. The results of these calculations agreed with that predicted by consideration of the theoretical maximum possible moment for each axial load ratio.

Another problem was discovered when these $M-P-\varnothing$ curves were used directly in the beam-column failure load program. None of the iterative solutions supply exact closed form solutions; they converge on a solution within an acceptable degree of error. Since some error is inherent in each iteration, the errors may accumulate. The degree of error is still small but it means for some cases of axial load with the curvature equal to zero, small positive or negative moments appear when they should be equal to zero. When the curves with these small negative moments were used in the failure load program it caused instability in the beam-column solution. It was discovered that the curve interpolation portions of the program could not handle negative numbers. The solution was to assign all the moments at zero curvature to exactly zero as they should be. The $M-P-\varnothing$ curve generation program could be changed to auto- 


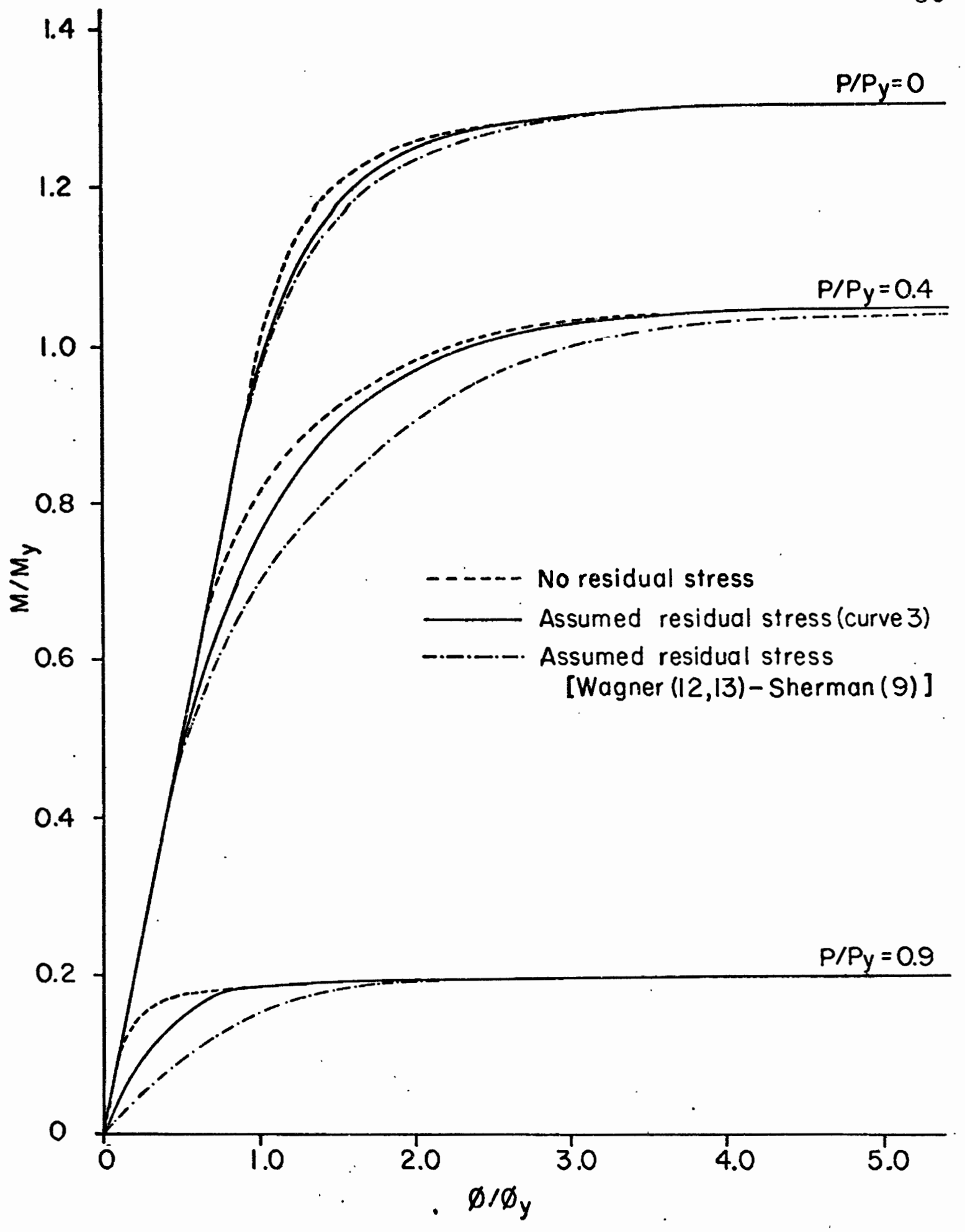

Figure 25. $M-P-\varnothing$ Curves from improved generation program 
matically assign moment equal to zero for curvature equal to zero which would eliminate the need to change the output later. In this case, the program was not changed since the magnitude of the moment gives an indication of the composite accuracy of the manipulations of the program.

It should be noted that the M-P- $\varnothing$ curves calculated for Wagner's $(12,13)$ residual stress distribution exhibit a flattening effect when compared to the curves calculated for the suggested residual stress distribution (Curve 3, Figure 18). This means when using Wagner's distribution the section is weaker than would be predicted using the suggested distribution (Curve 3, Figure 18). It should also be noted that different axis orientations control the flattest (most critical) curve at different axial load ratios.

It should be noted that the M-P- $\varnothing$ curves generated using the suggested residual stress distribution give more consistent results with respect to the controlling axis orientation for different slenderness ratios than by using the distribution suggested by Wagner $(12,13)$.

Once the M-P- $\varnothing$ curve generation and interpolation problems had been solved, the failure load program was used for the same sections and loading conditions that wagner (13) used. M-P- $\varnothing$ curves were generated for five axes orientations; $0^{\circ}, 45^{\circ}, 90^{\circ}, 135^{\circ}$, and $180^{\circ}$ with respect to the reference axis (perpendicular to weld axis), for Wagner's residual stress distribution and for the suggested 
residual stress distribution. Each of these $M-P-\varnothing$ curves were used in the beam-column failure load program. This was done since the $M-P-\varnothing$ curves for Wagner's residual stress distribution indicated different axes orientations controlled for different $\mathrm{P} / \mathrm{Py}$ ratios. The controlling results were then graphed as a failure load curve using the same format as shown by sherman (9.).

Figure 26 shows the resulting curve for Wagner's distribution at an orientation of $90^{\circ}$ with respect to the reference axis.

The failure load results for the suggested residual stress distribution are shown in Figure 27 since the curves are close enough to those of Figure 26 as to make representation on the same figure difficult at this scale. The orientation of the axis for the suggested residual stress distribution (Curve 3 Figure 18 ) is $180^{\circ}$ with respect to the reference axis. These curves were compared to available test data and reasonable agreement was indicated. 


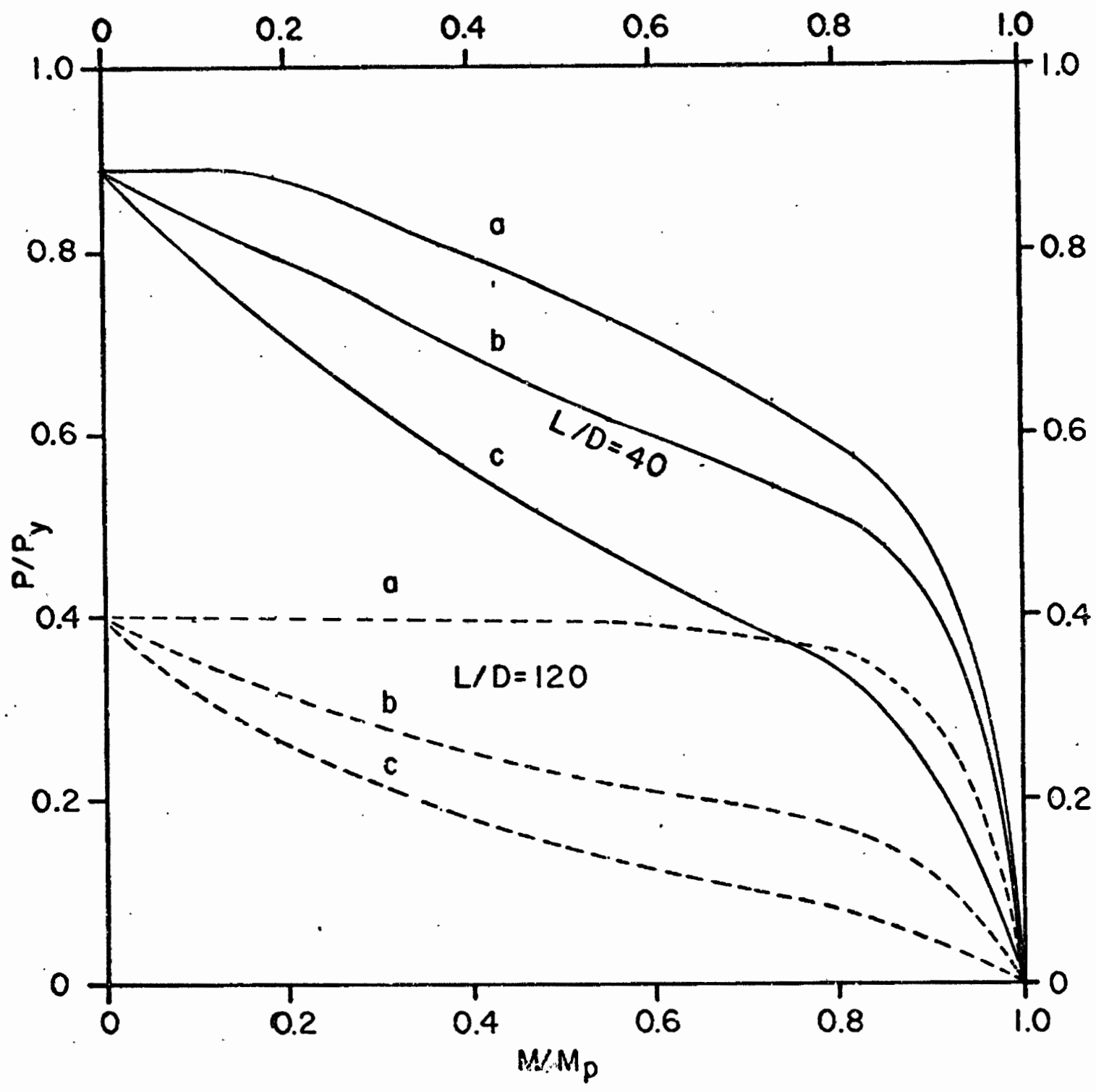

a Double curvature

b Single end moment

c Single curvature

Figure 26. Beam column results - Wagner's distribution 


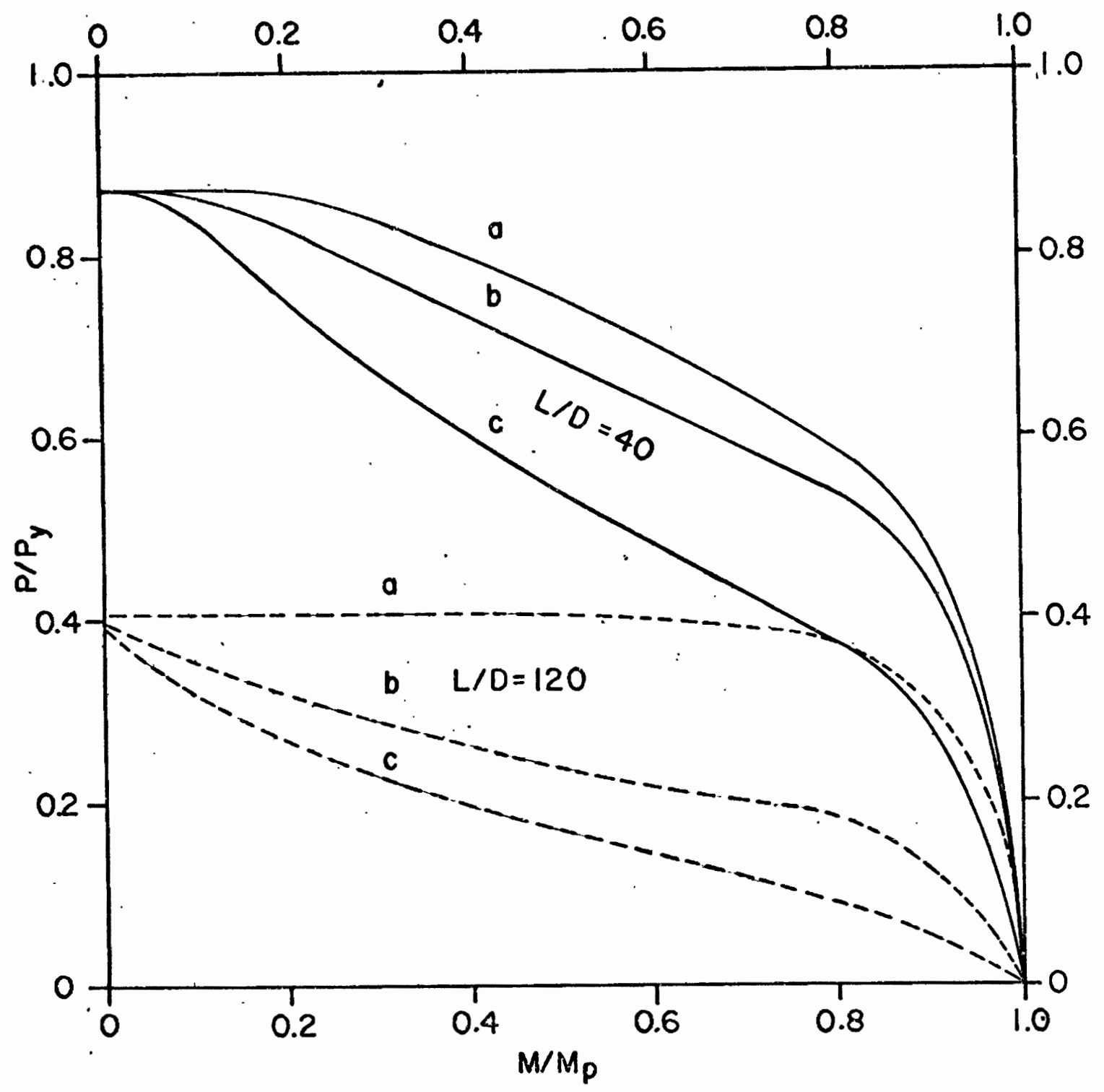

a Double curvature

b Single end moment

c Single curvature

Figure 27. Beam column results-curve 3 (Fig. 18). 
CHAPTER VI

CONCLUSIONS

Comparing the balanced residual stress curve (Curve 3) with the curves determined by the sectioning method, hole drilling method and Wagner's assumed distribution, the balanced curve suggested for use (Curve 3 Figure 18) appears to be a smooth curve more closely modeling the test data (Fig. 28). Figure 29 shows a comparison of $M-P-\varnothing$ curves for no residual stress, Wagner's distribution, that deduced from member behavior and the balanced residual stress curve (Curve No. 3). Sherman (9) presents a curve showing the effect of residual stress on strength under axial load which is shown in Figure 30 with the points determined using the residual stresses from Curve 3 (Figure 18) added to it. Using the same data as Sherman used in the beam column failure load program but with the balanced residual stress distribution of Curve 3 (Figure 18) instead of Wagner's curves, new points are determined which are plotted on Sherman's Figure 6 to show the effect of the balanced distribution (Fig. 31).

While there are large differencesin the shape of the M-P- $\varnothing$ curves as previously described (Figure 25,29), the effect on the interaction curve for failure loads is 


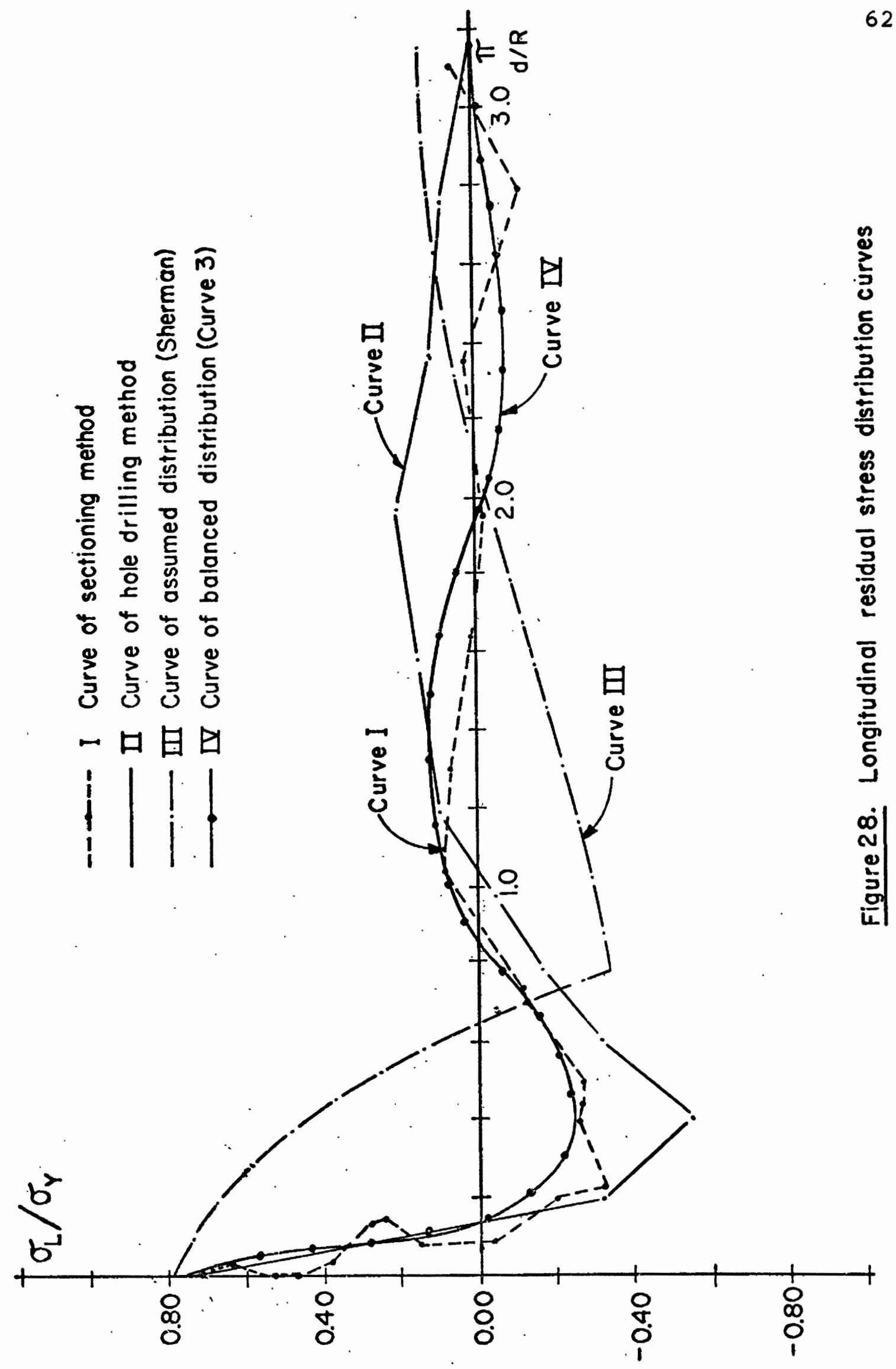




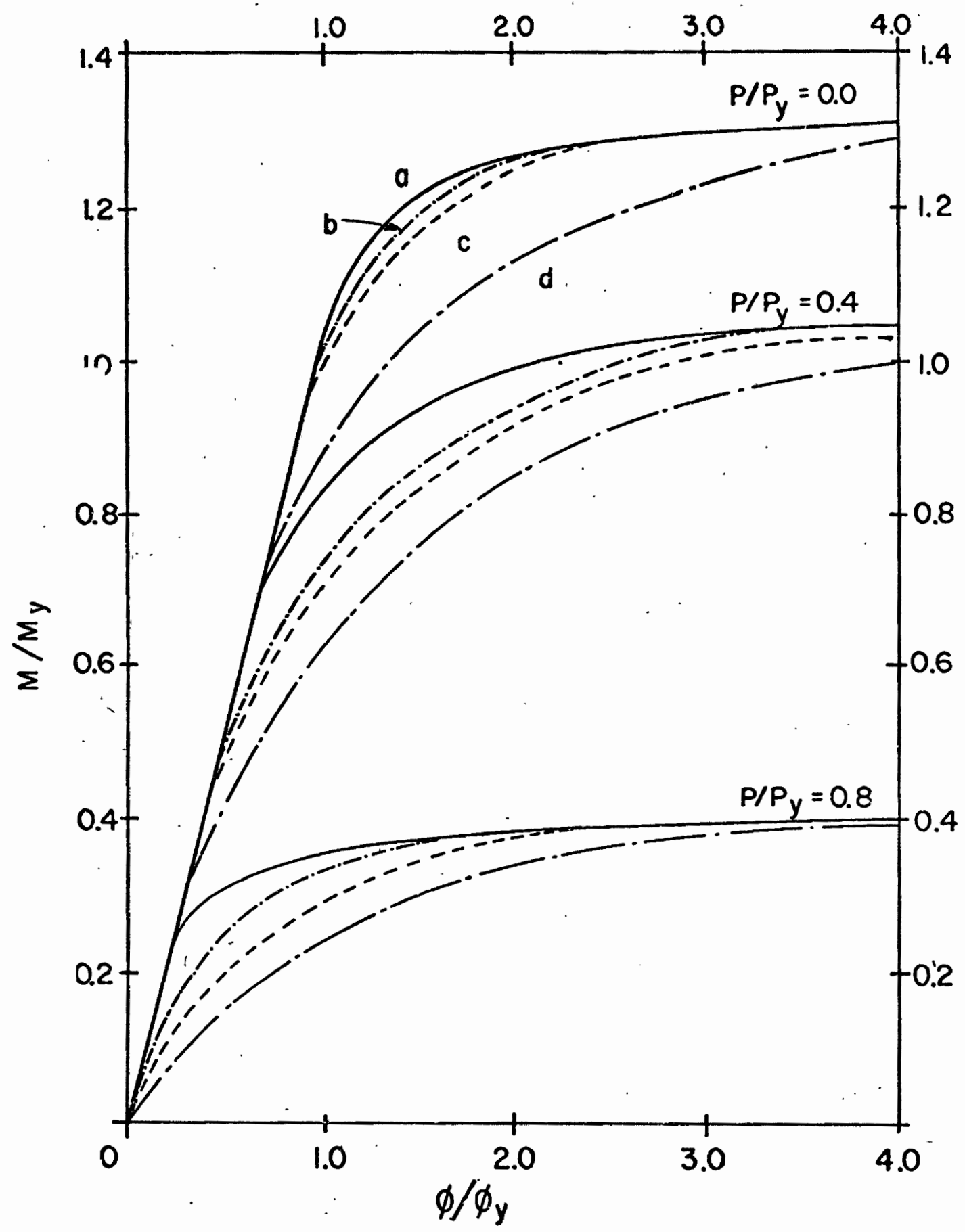

a. No residual stress

b. Assumed residual stress of curve 3

c. Assumed residual stress, Sherman

d. Deduced from member behavior

Figure 29. Moment-curvature relationships 


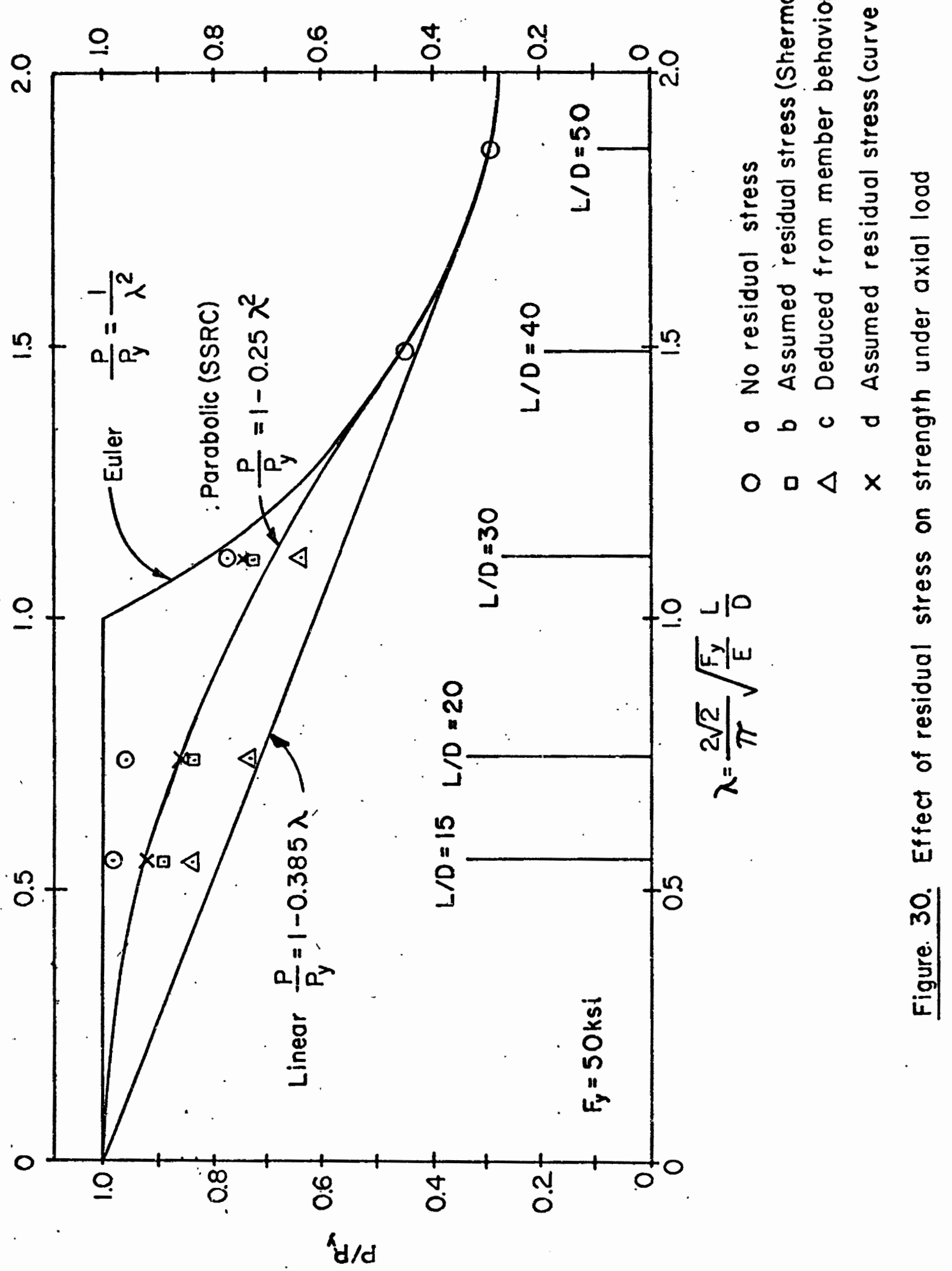




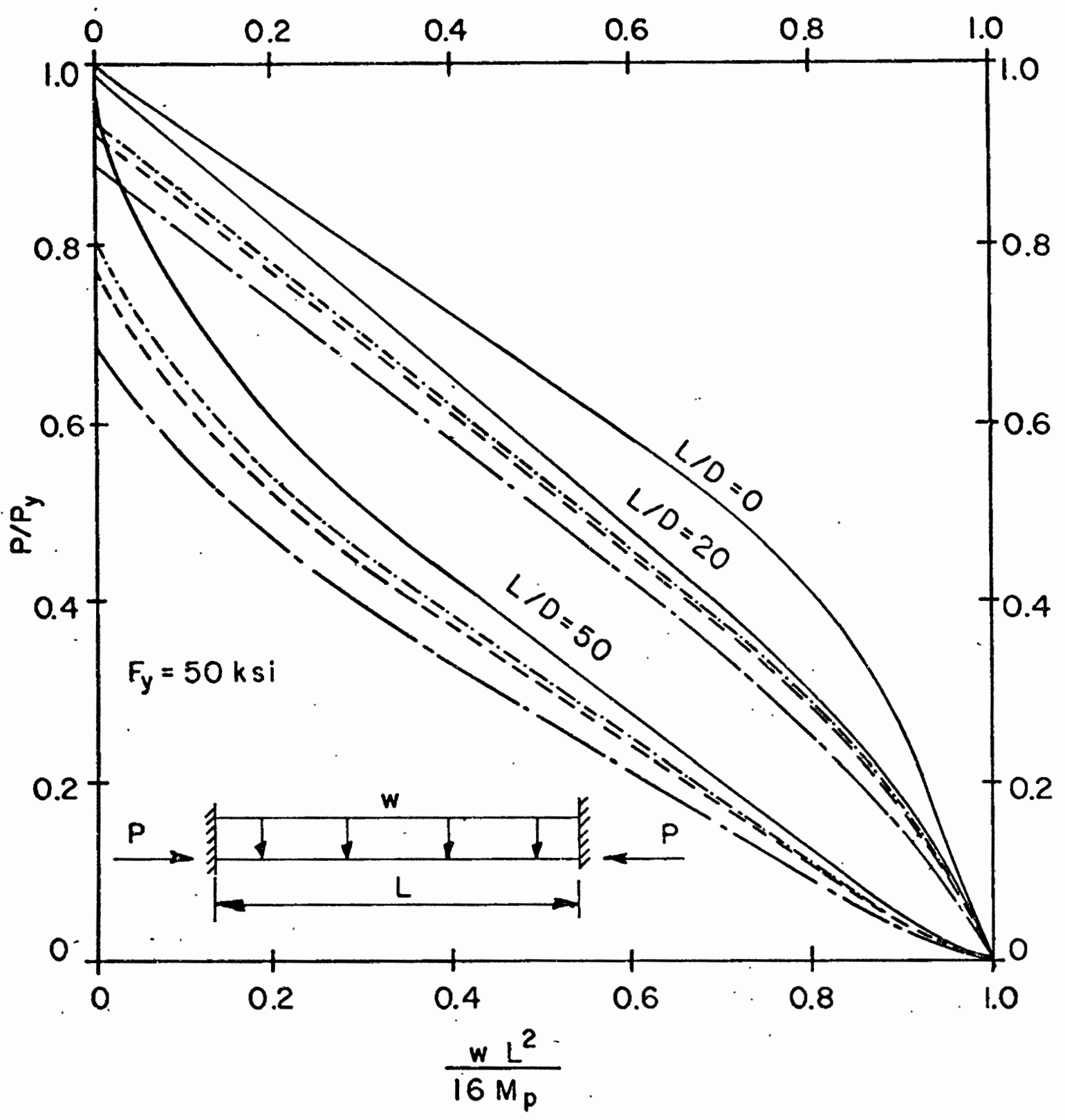

- a No residual stress

-

- c Deduced from member behavior

-........ o Assumed residual stress distribution (curve 3)

Figure 31. Effect of residual stress 
slight. The maximum difference between predicted failure loads is in the neighborhood of five percent. The failure load curve as described from $M-P-\varnothing$ curves using the suggested residual stress distribution shows an increase in predicted strength over the failure load curve using the M-P- $\varnothing$ curves as described by Wagner's residual stress distribution (Figure $26,27,30,31$ ). This agrees with the prediction based on the shape of the $M-P-\varnothing$ curves (Figure 25,29$)$. The interaction curve obtained using the M-P- $\varnothing$ curve generated based on the suggested residual stress distribution (Curve 3 Figure 18) does not exactly conform to the test data as plotted but that is to be expected for several reasons. One is the possible error in the physical test data, another is the small errors that are inherent in the process of curve interpolation and iterative solution for axial load, moment and failure load; another is the use of a perfect bilinear stress-strain curve instead of using tabulated data from physically testing a coupon from the member tested. The degree of agreement is acceptable in light of these considerations and the use of the suggested residual stress distribution is recommended for future research into the behavior of welded steel tubes in combined bending and axial load.

It is fur.ther recommended that the $M-P-\varnothing$ curve generation program be changed to facilitate more rapid use of the program. A data file should be created that would 
contain the stresses and strains on each element that result for each axial load ratio used. These stresses and strains can be obtained using the first portion of the M-P- $\varnothing$ curve generation program as re-written in this study. The stresses and strains that result from the application of residual stress plus axial stress after the moment imbalance has been resolved are the ones that should be saved. By storing these stresses and strains, the program would not be required to recalculate them for each axis rotation thereby reducing the computer time used.

summarizing, the residual stresses present in a welded steel tube can be modeled by a smooth curve that describes stresses such that the cross section is in equilibrium.

Curve 3 (Fig. 18) models the existing test data well, is in equilibrium and exhibits consistent behavior in the $M-P-\varnothing$ and beam column failure load program. This curve is suggested for use in further research on the behavior of steel tube columns. The effect of these residual stresses on the predicted failure load of tubular beam columns is significant and should not be ignored. The difference in failure load predicted by this family of curves was five percent at the maximum, indicating that the exact shape of all parts of the residual stress curve is not required to obtain good results. Because of the large strength reductions indicated by use of the residual stress 
distribution described by Curve 3 (Figure 18) in the beamcolumn failure load program, it is evident that residual stresses can not be ignored when calculating failure loads in steel tube beam columns. 
REFERENCES

1. "Plastic Design in Steel, A Guide and Commentary," American Society of Civil Engineers 2nd Edition, 1971 .

2. Beedle, L. S., "Plastịc Design of Steel Frames", John Wiley and Sons, Inc., 1958.

3. Chen, W. F. and Ross; D. A., "Residual Stress Measurements in Fabricated Tubular steel Columns," Fritz Laboratories Report, No. 393.4, July 1975.

4. Chen, W. F. and Ross, D. A., "Tests of Fabricated Tubular Columns," Journal of the Structural Division, Proceedings of ASCE, Vol. 103, No. ST3, March 1977.

5. Disque, R. O. "Applied Plastic Design in Steel" Van Norstrand Reinhold Company, 1971.

6. Dwyer and Galambos, "Plastic Behavior of Tubular Beam-Columns", Journal of the Structural Division, ASCE, Volume 91, ST4, August 1975.

7. Marshall, P. W., "Stability Problems in Offshore Structures", presentation at the Annual Technical Meeting of the Column Research Council, St. Louis, March 25, 1970.

8. Salmon, C. G. and Johnson, J. E., "Steel Structures, Design and Behavior" Intext Educational Publishers 1972 .

9. Sherman, D. R., Erzurmlu, H., Mueller, W., "Behavioral Study of Tubular Beam Columns." Journal of the Structural Division, ASCE, Volume 195, No. ST6, June 1979.

10. Szuladzinski, G., "Moment-Curvature for Elastoplastic Beams," Journal of the Structural Division, Proceedings of ASCE, Vol. 101, No. ST7, July 1975 . .

11. Tran, C. M., "An Investigation of the Hole-drilling Technique for Measuring Residual Stresses in Welded Fabricated steel Tubes" Masters Thesis, Portland State University, Dec. 1977. 
12. Wagner, A. L., Mueller, W. H. and Erzurmlu, H., Portland State University, "Design Interaction Curves for Tubular Steel Beam-Columns", Eighth Annual Offshore Technology Conference, Houston, Texas, May 3-6, 1976.

13. Wagner, A. L., "A Numerical Solution for the Ultimate Strength of Tubular Beam-Columns," Masters Thesis, Portland State University, Nov. 1976. 
APPENDIX I

CURVE BALANCING COMPUTER PROGRAM 


\section{CURVE BALANCING PROGRAM}

\section{DATA INPUT}

NOTE: Use of the punch output option gives the user a deck of cards with the stress-strain information on them in proper form for direct input to the moment-thrust curvature program (average over element). Numbers at left indicate card columns.

A. Control Data; Curve Identity Number.

FORMAT (2I3)

1-3 Curve identity number

4-6 Are stress-strain values to be punched on cards? (- or 0 no, + yes)

B. Control Data.

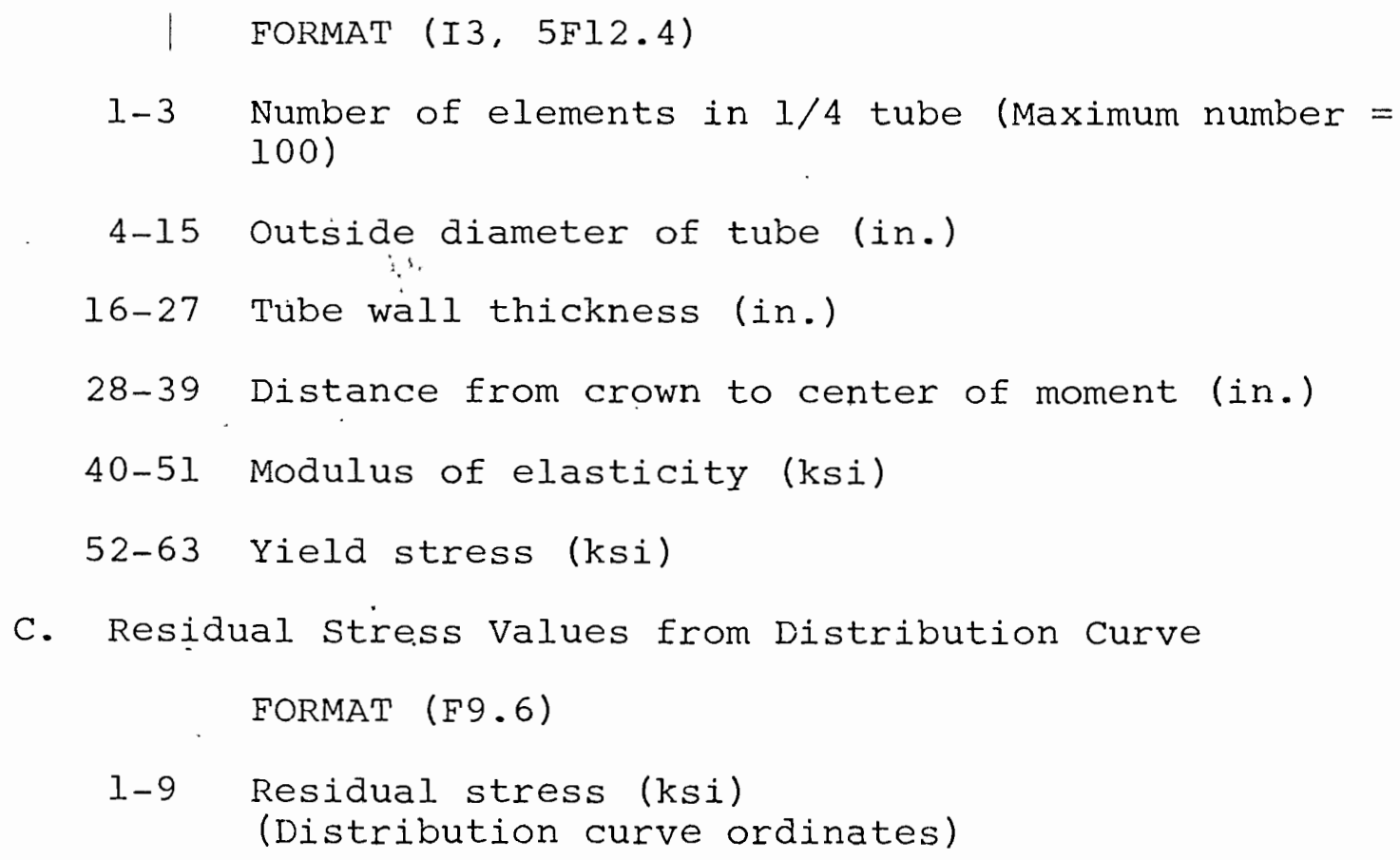
100)

4-15 Outside diameter of tube (in.)

16-27 Tủbe wàll thickness (in.)

28-39 Distance from crown to center of moment (in.)

40-51 Modulus of elasticity (ksi)

52-63 Yield stress (ksi)

C. Residual stress Values from Distribution Curve FORMAT (F9.6)

1-9 Residual stress (ksi)

(Distribution curve ordinates) 
FLOW DIAGRAM

RESIDUAL STRESS DISTRIBUTION CURVE BALANCING

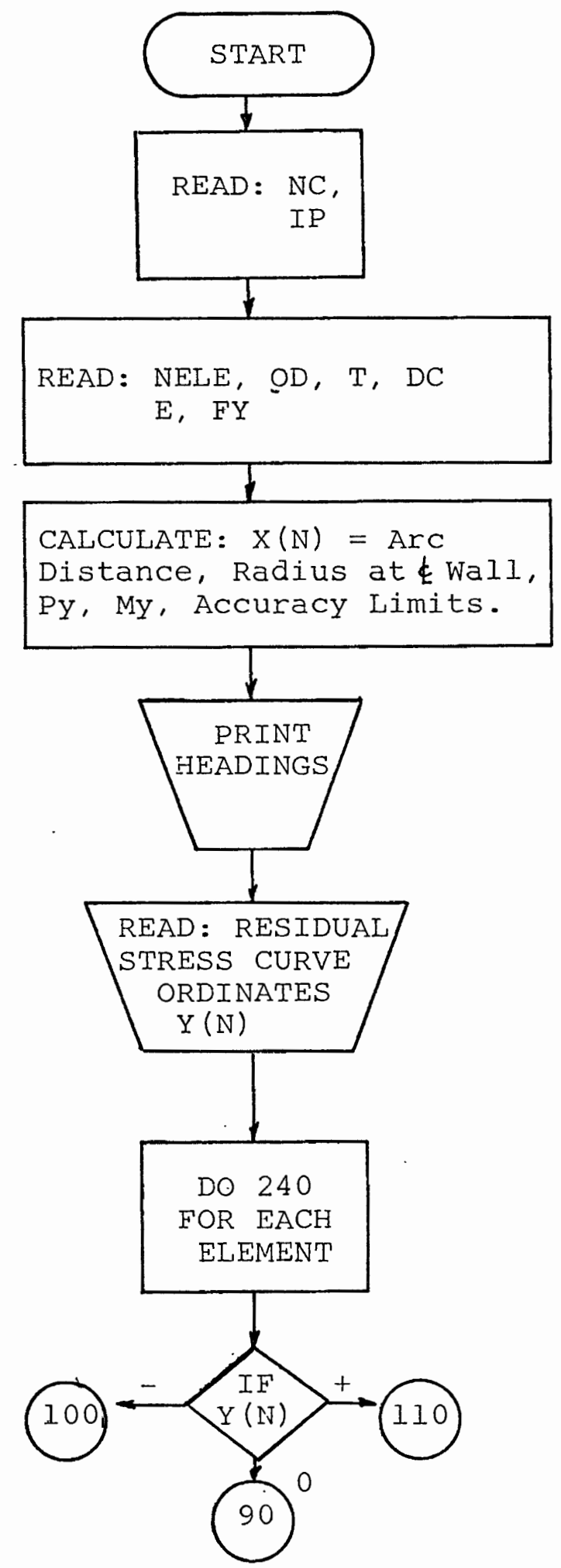




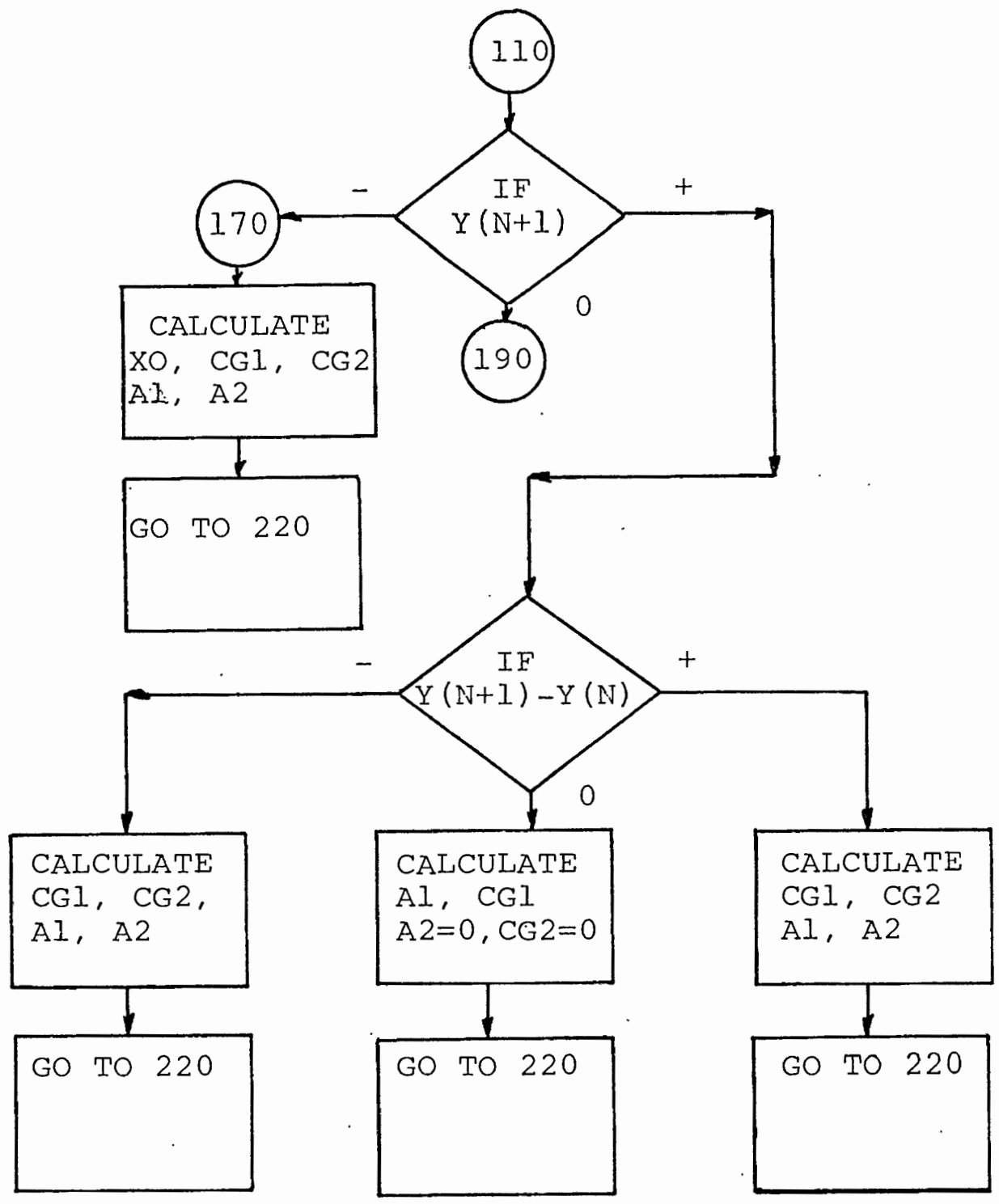



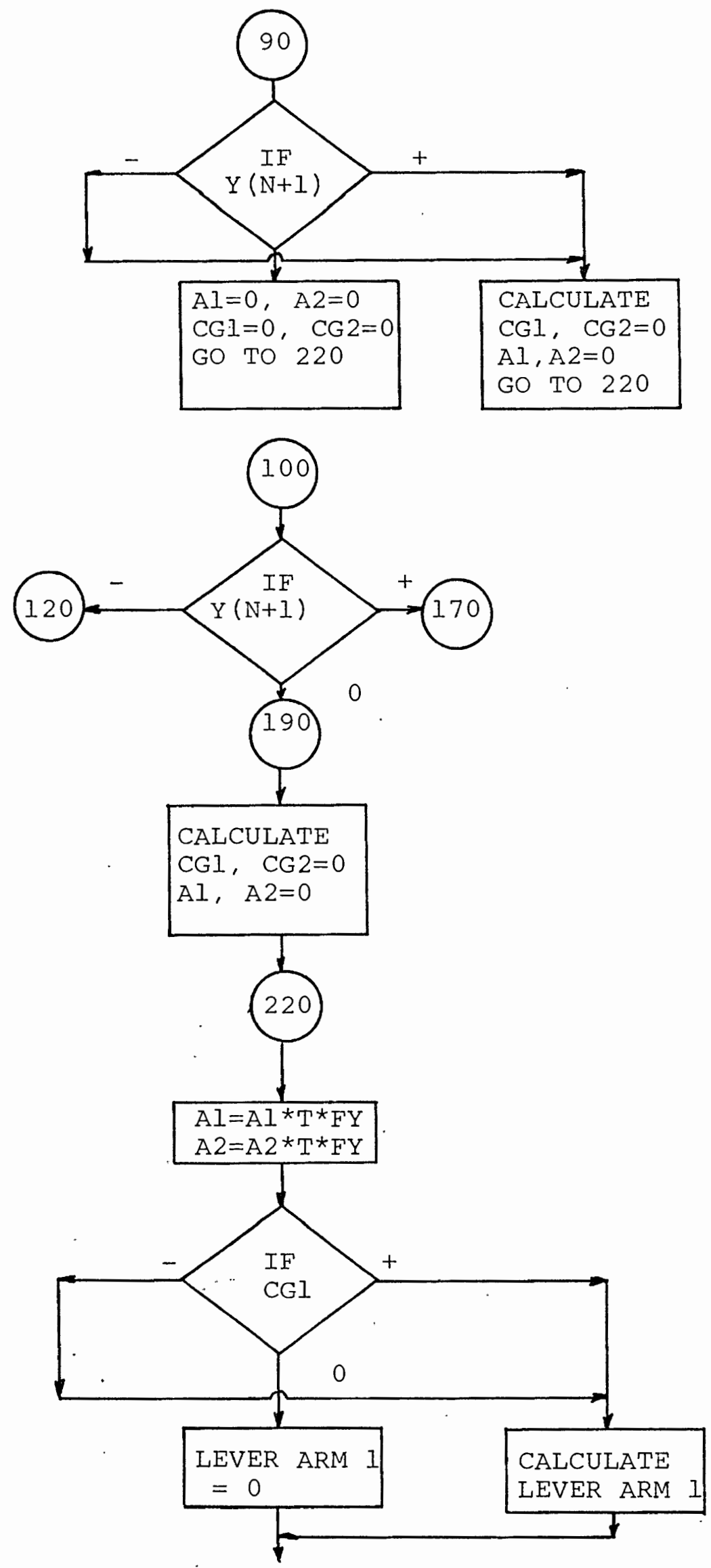


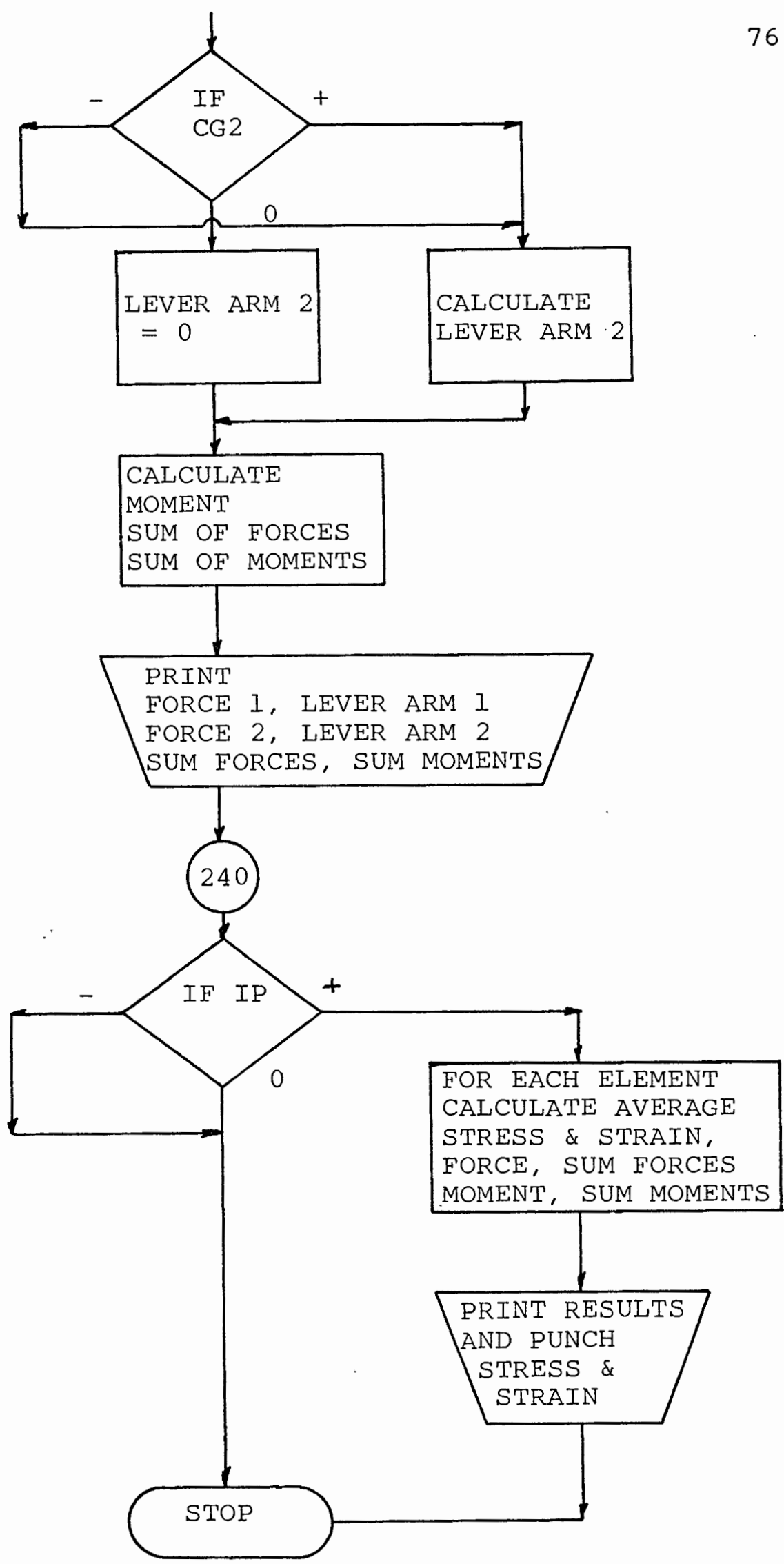


$=$

$\because z$

$\sum_{i}$

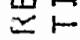

$x \cdot E u$

造的的

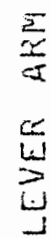

以可岂

$\geq \omega$ i

wis 2

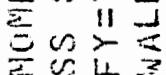

然边

$\sum_{\alpha}$

थ造吉的

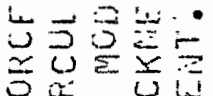

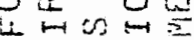

00

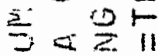

$\vec{n} \geq \stackrel{5}{0}-\frac{1}{0}$

c

5 为政

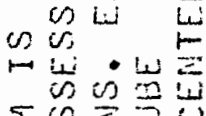

$\Sigma \sim \sum \equiv$

सن

난안

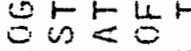

2 $10 \%$

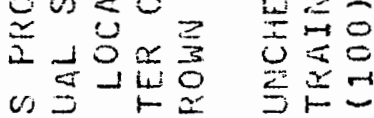

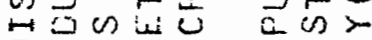

$=\omega_{0} \infty$

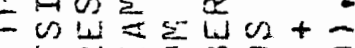

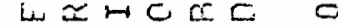

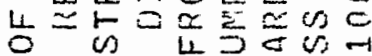

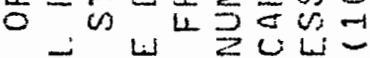

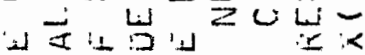

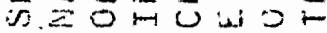

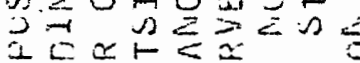

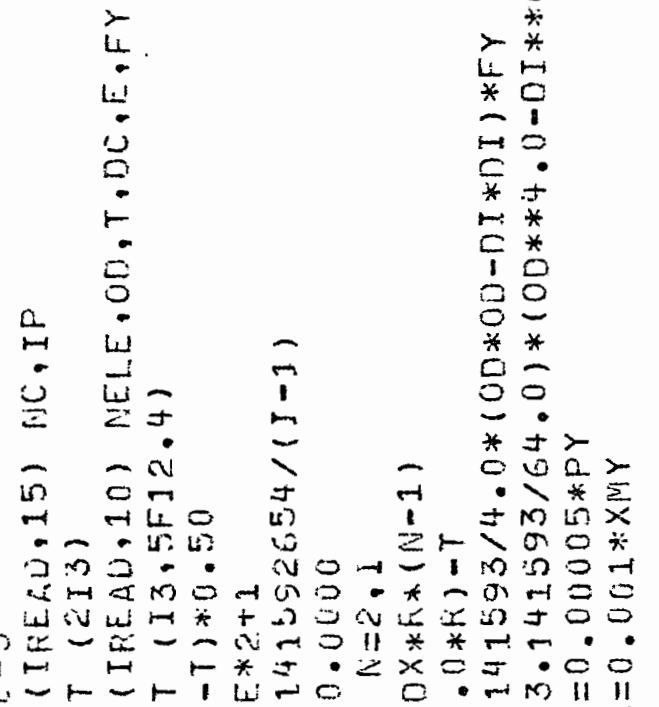

$\begin{array}{ll}2 & w \\ \vdots & 0\end{array}$

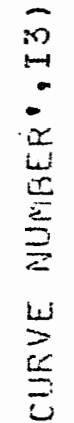

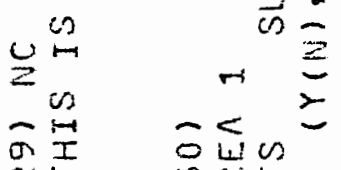

昰

造 is

w

\lrcorner$=$

吾京

NF $\quad$ 兵U⿺

wi

$5 * *$ 占 $\simeq$ ๔... $\pm=0=$

$\circ \cdot$

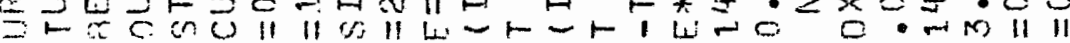

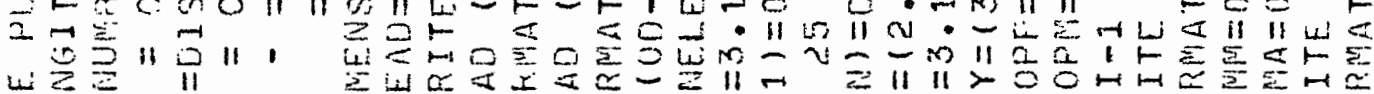
IE F-

$$
\therefore 2
$$

n 


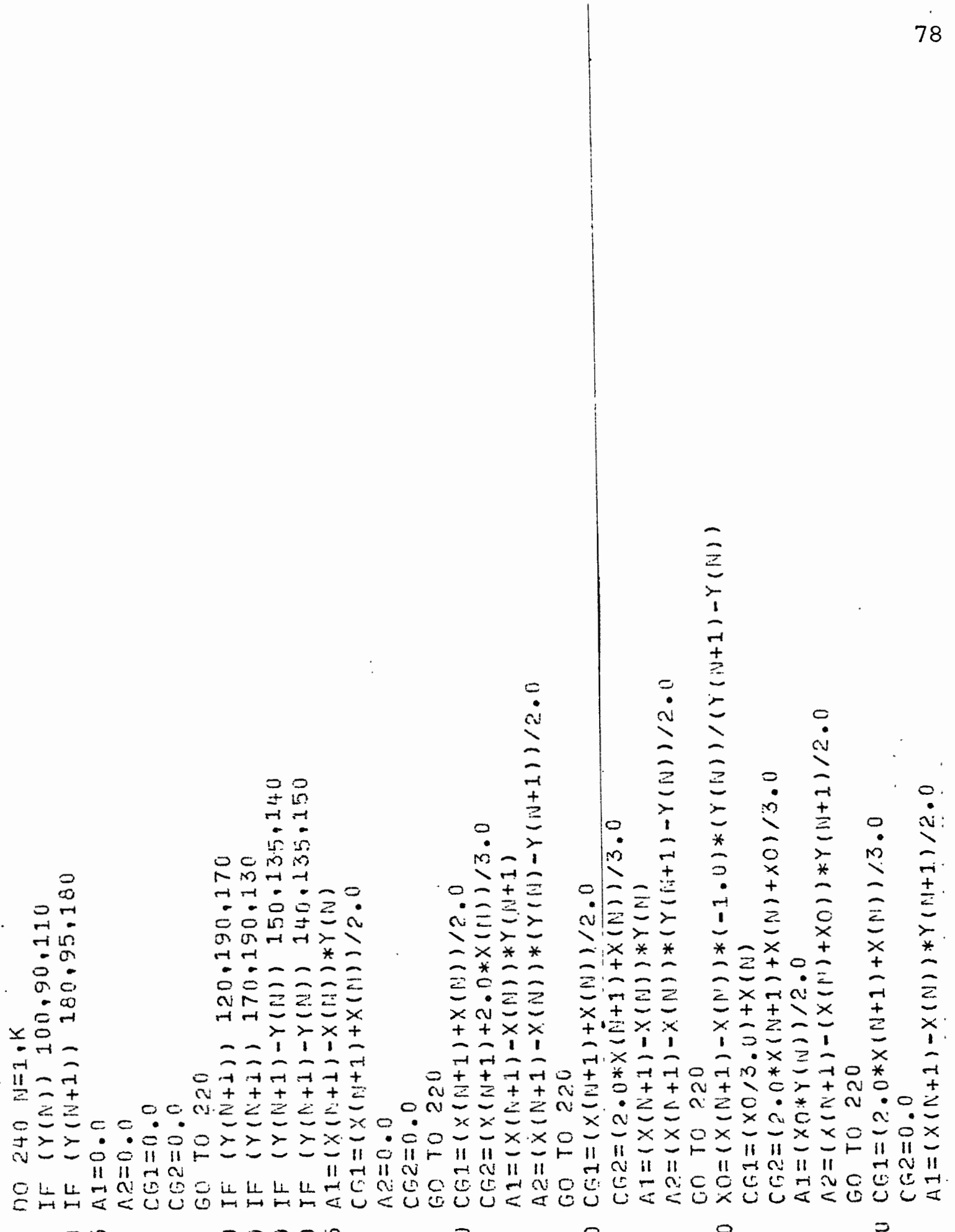




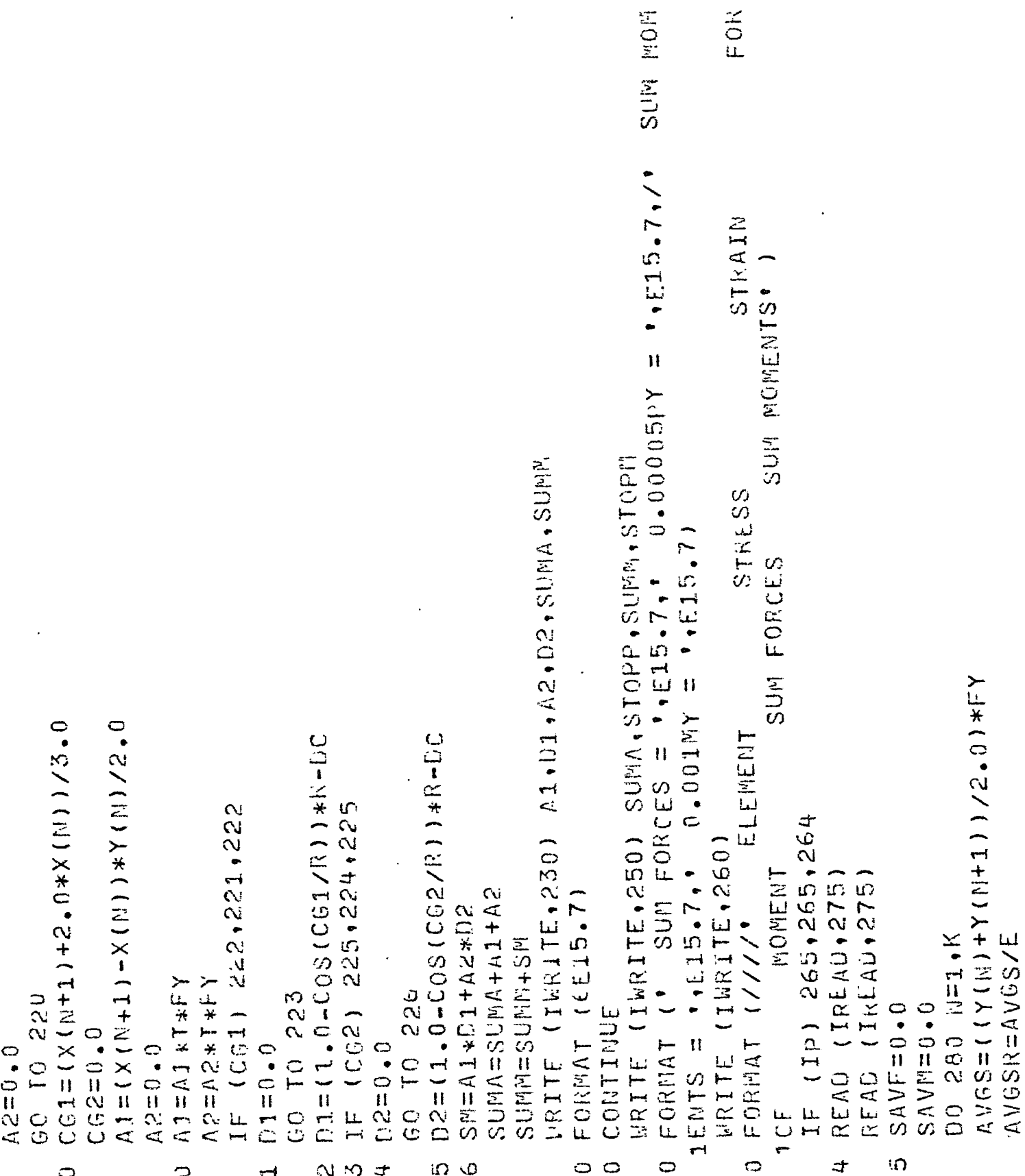

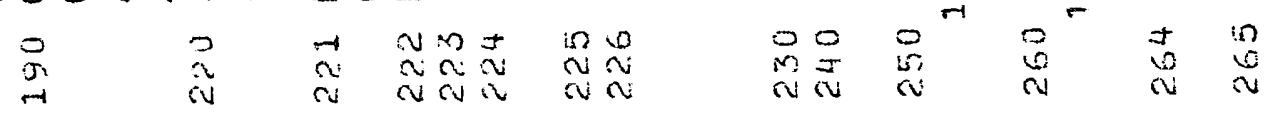

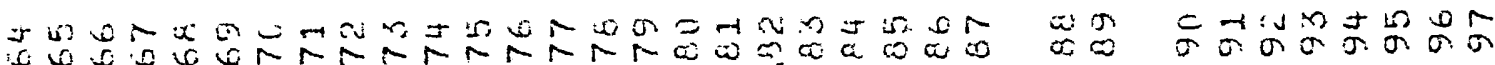




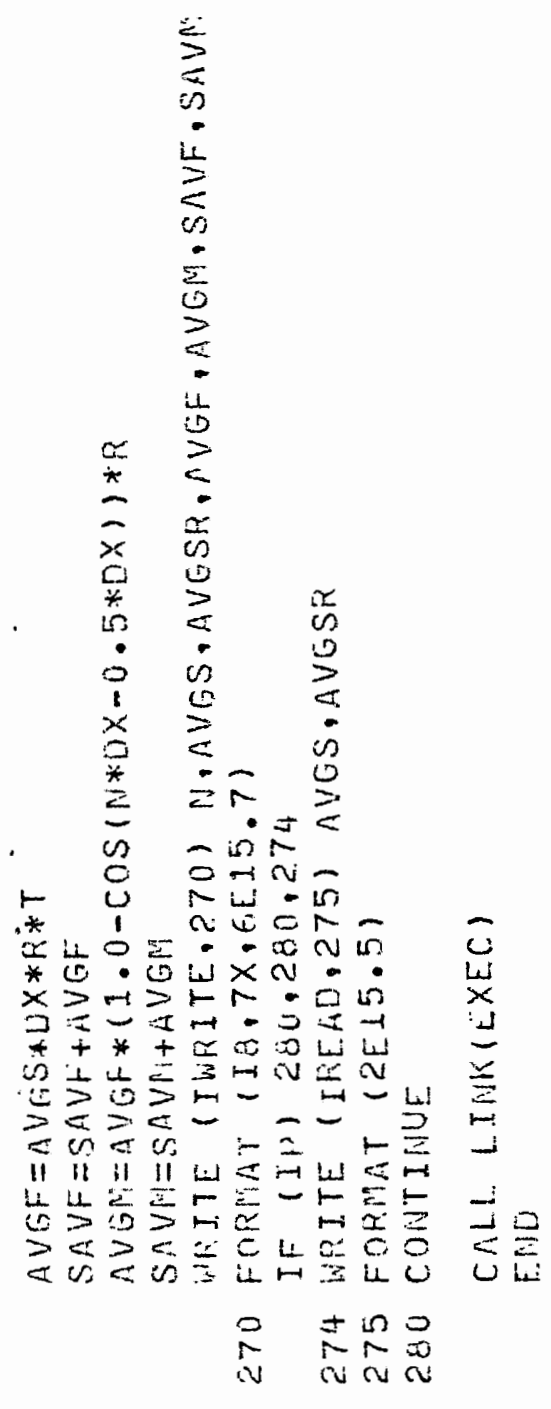

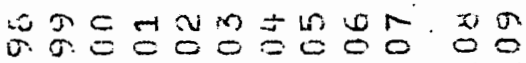
ननमन नलम नल 


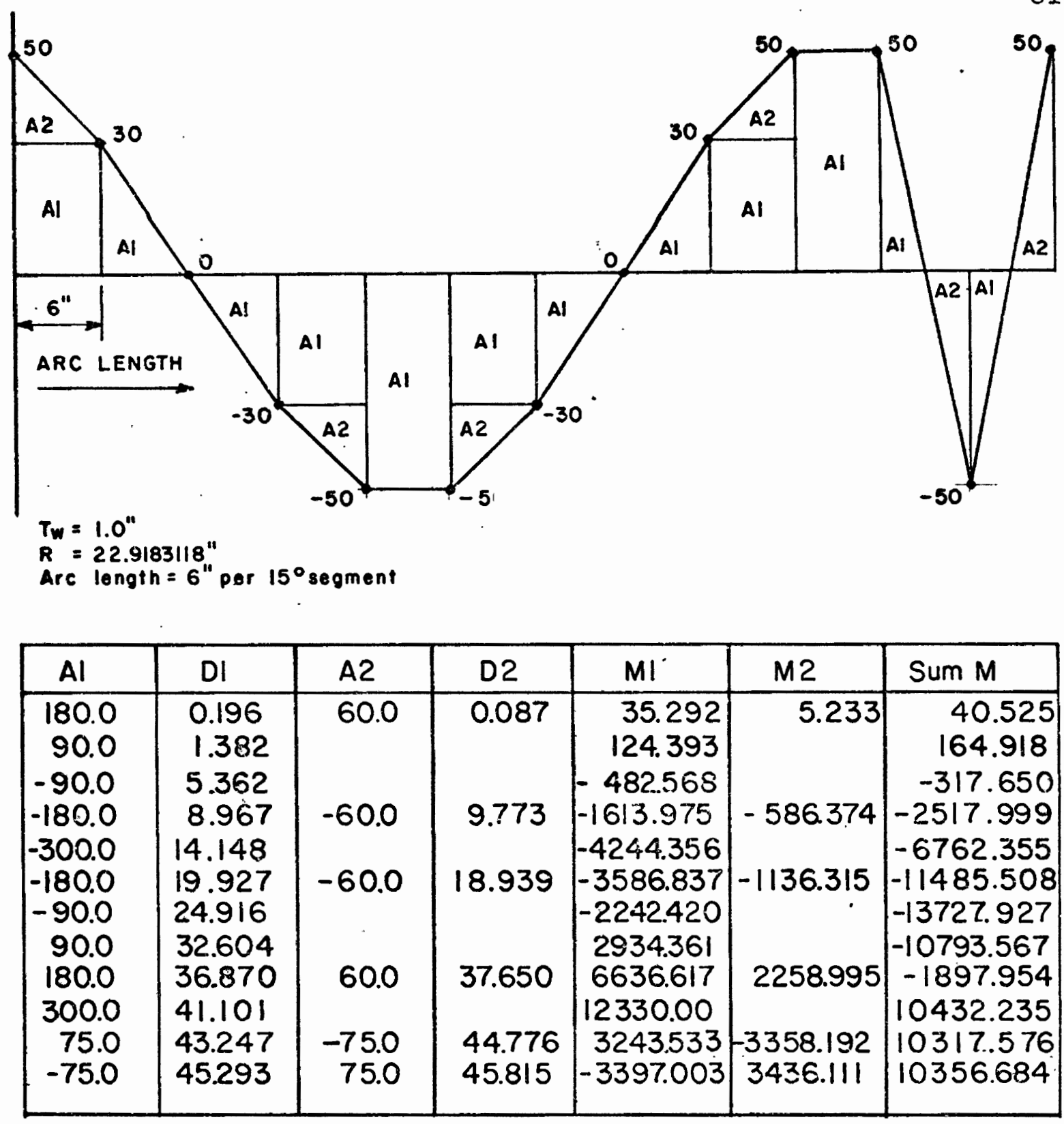

Compare with program output (next page)

Figure 32. Example for testing curve program 


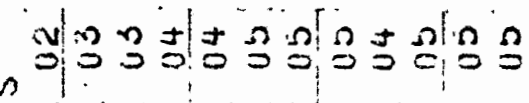

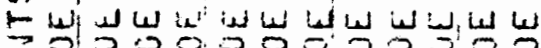
स. 000.90000000

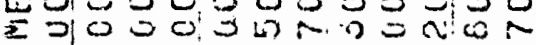

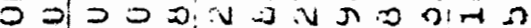

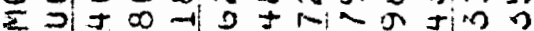

- Sid

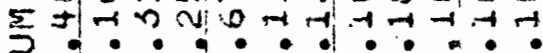

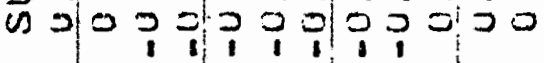

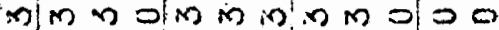

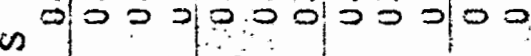
Uإ

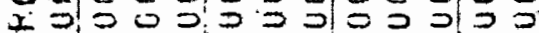
吉

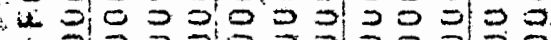

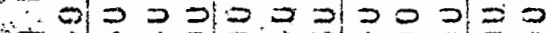

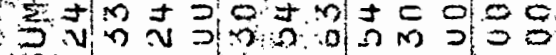
$\therefore \circ \dot{0}+0 \dot{0}=\dot{0}=\dot{0}=\dot{0}$ 1.: 110

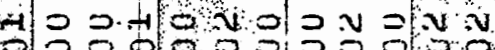

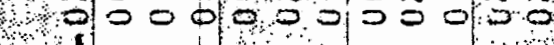

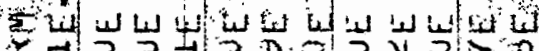

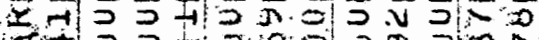

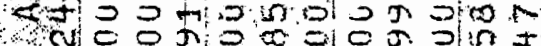

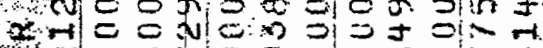

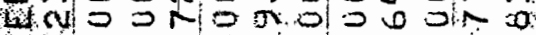

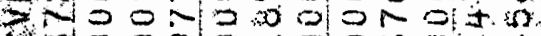

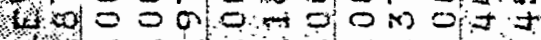

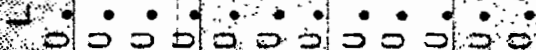

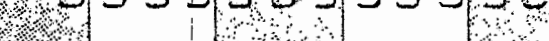
\%

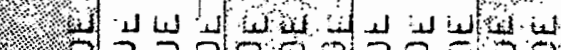

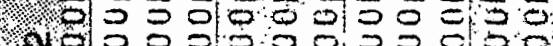

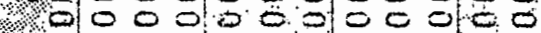

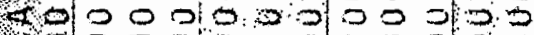

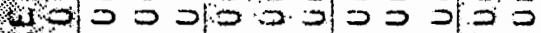

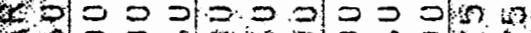

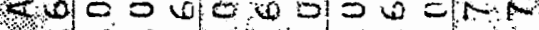

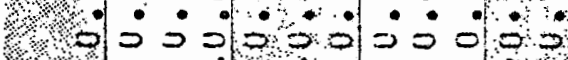
\%ै। 1 किस: +ैo

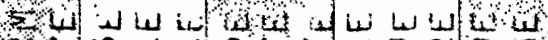

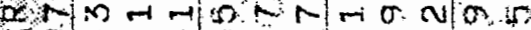
40 J $\mathrm{m}$ man 00000

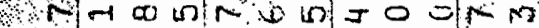

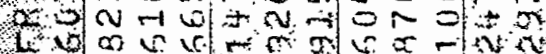

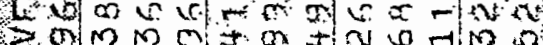

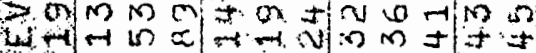

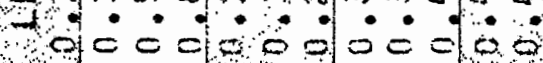
अै।

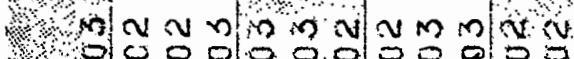

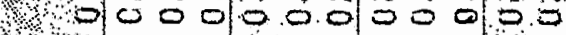
अیا

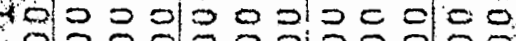

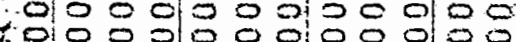
山.

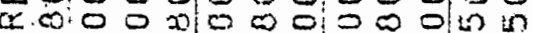

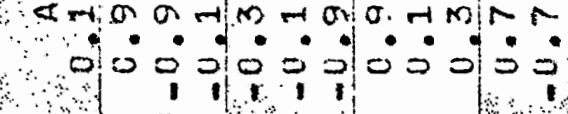
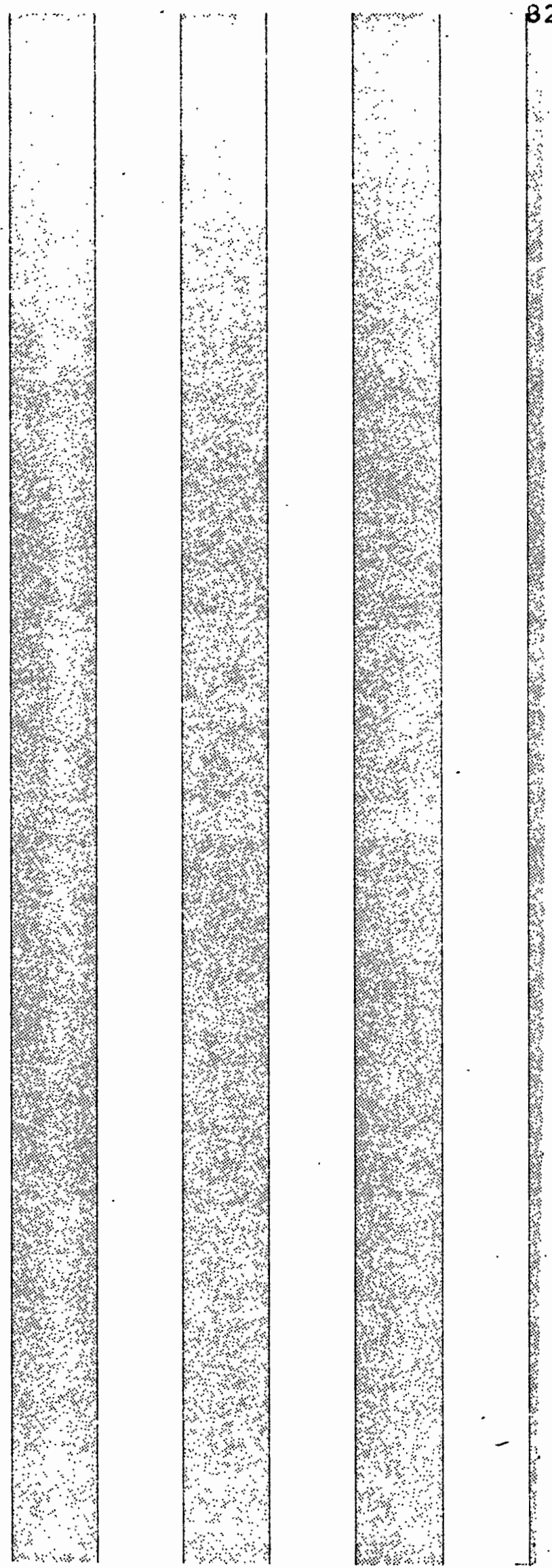
APPENDIX II

REVISIONS TO MOMENT-THRUST-CURVATURE PROGRAM 
MOMENT-THRUST-CURVATURE PROGRAM

\section{DATA INPUT}

NOTE: The last data card must assign the outside diameter a value of zero to stop the program.

Numbers at left indicate card columns.

A. Control Data; Cross Section and Material Properties. FORMAT (4I5, 4E15.5)

1-5 Actual stress-strain data used? ( $+=$ Yes; $-I=$ No)

6-10 Residual stresses used? $(+1=$ Yes ; $-I=$ No $)$

11-15 Number of layers of elements. $(\operatorname{Max} .=5)$

16-20. Number of elements in $1 / 4$ circle of one layer. (The product of the last two numbers must not exceed 50.)

21-35 Outside diameter (in.)

36-50 wall thickness (in.)

5l-65 Modulus of elasticity. (ksi)

66-80 Yield stress. (ksi)

B. Data and Time of Run

FORMAT (4I5)

1-5 Month.

6-10 Day

11-15 Year

16-20 Time (001 - 2400) 
C. Control Data

$$
\text { FORMAT (7I5) }
$$

1-5 Number of $P / P Y$ values

$(\operatorname{Max} .=12)$

6-10 Number of PHI/PHIY values

$(\operatorname{Max} .=25)$

11-15 KSKIP

( $1=1$ stress-strain data for one half tube)

( =2 stress-strain data for entire tube)

16-20 Rotation of bending axis

(Number of elements)

21-25 Curve identification number

26-30 Are curves to be plotted?

$(-$ or. $0=$ No)

$(+\quad=$ Yes $)$

31-35 Are M-P-PHI values to be punched on cards?

$\begin{aligned}(- \text { or } 0 & =\text { No }) \\ (+\quad & =\text { Yes })\end{aligned}$

D. Axial Load Values

FORMAT $\cdot(6 \mathrm{~F} 10.5)$

1-10 P/PY values (Always Positive)

$11-20$

$21-30$

$31-40$

$41-50$

$51-60$

E. Curvature Values

FORMAT (5F10.5,/,5F10.5,1,5F10.5, 1,5F10.5,1,5F10.5)

1-10 PHI-PHIY values (Always Positive)

$11-20$

$21-30$ 
$31-40$

$41-50$

NOTE: The data for one problem is now complete if the actual stress-strain data and residual stresses are not used. If both options are used, the stress-strain curve data is read in first.

F. Stress-Strain Curve Data

1. Control Card FORMAT (I5)

1-5 Number of tabulated points on stress-strain curve.

2. For each tabulated point FORMAT (2E15.5)

1-15 Stress value

16-30 Strain value

G: Residual Stress Data

1. Time of Residual Stress Calculation FORMAT (4I5)

1-5 Month

6-10 Day

11-15 Year

16-20 Time

2. For each element FORMAT (2E15.5)

1-15 Stress value 16-30 Strain value 
FLOW DIAGRAM

CALCULATION OF MOMENT-THRUST-CURVATURE DATA

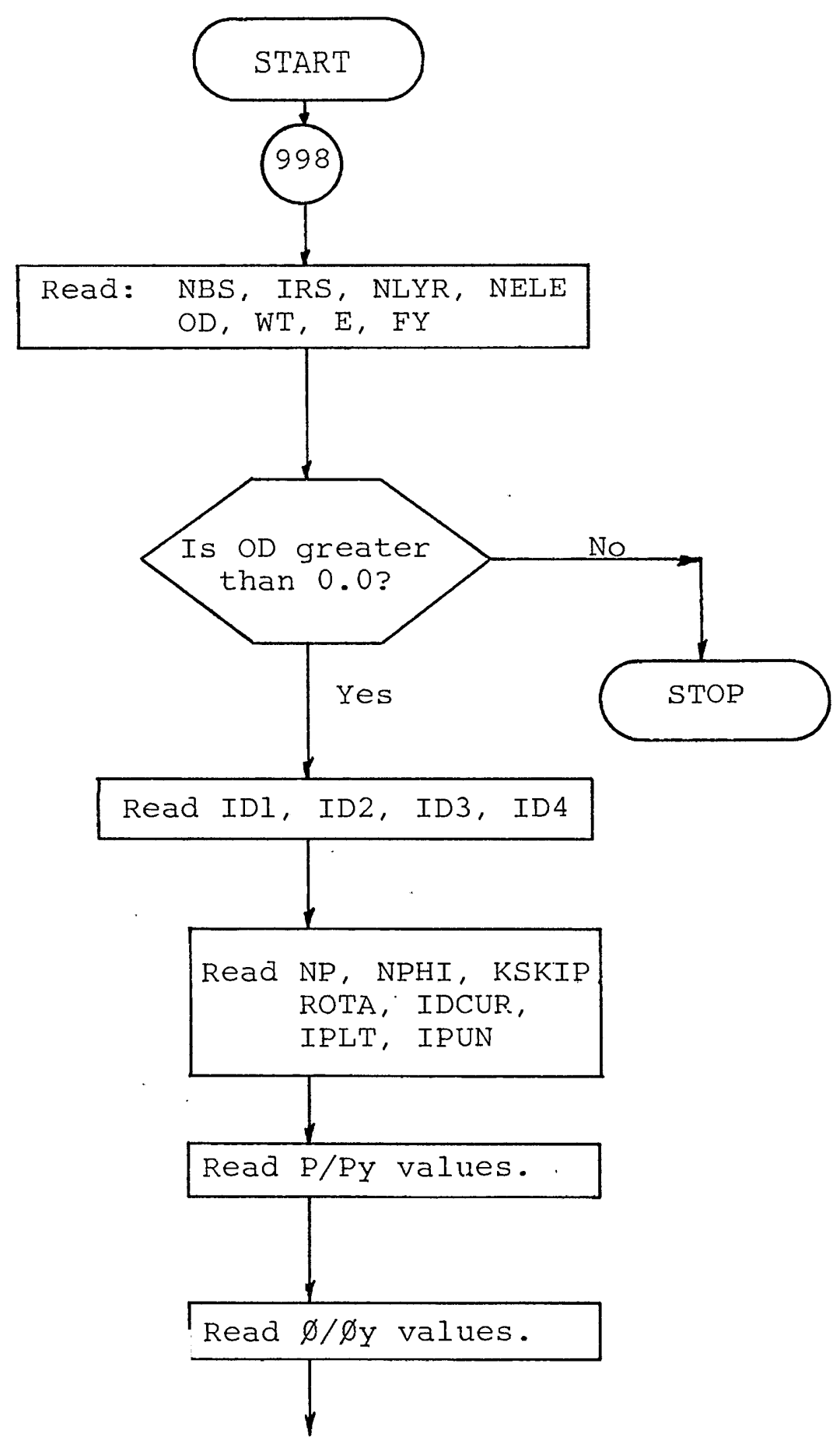




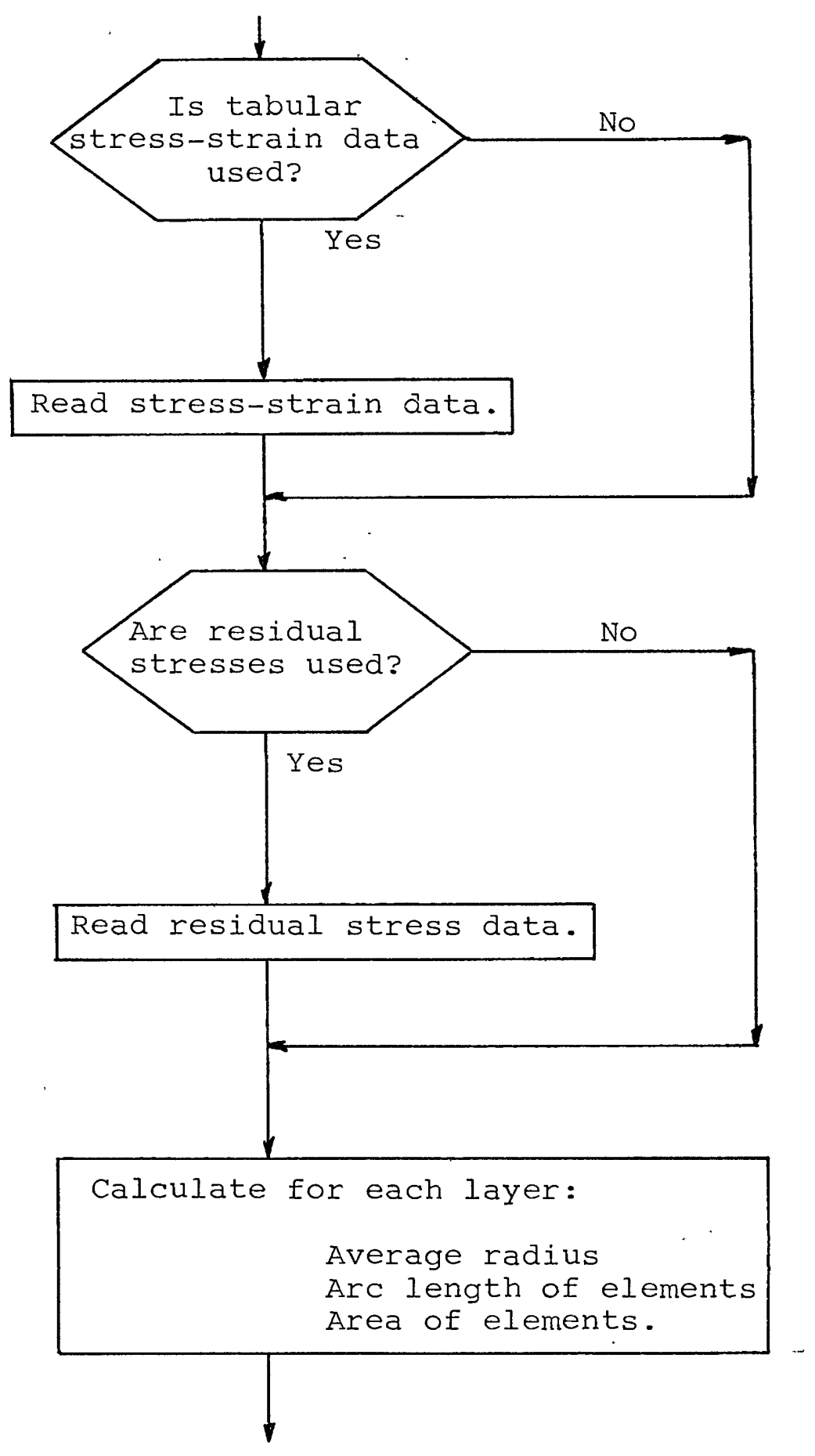




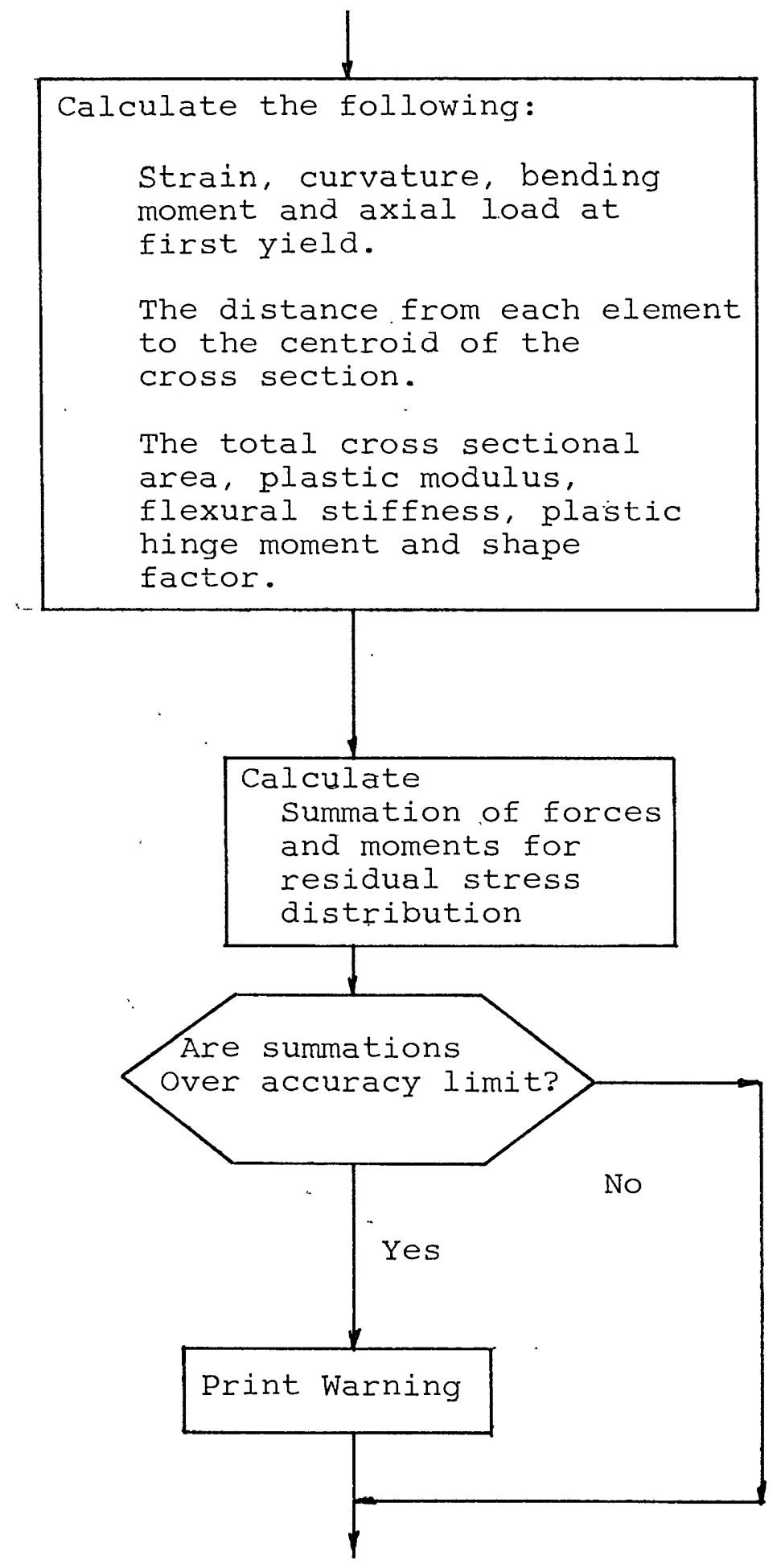




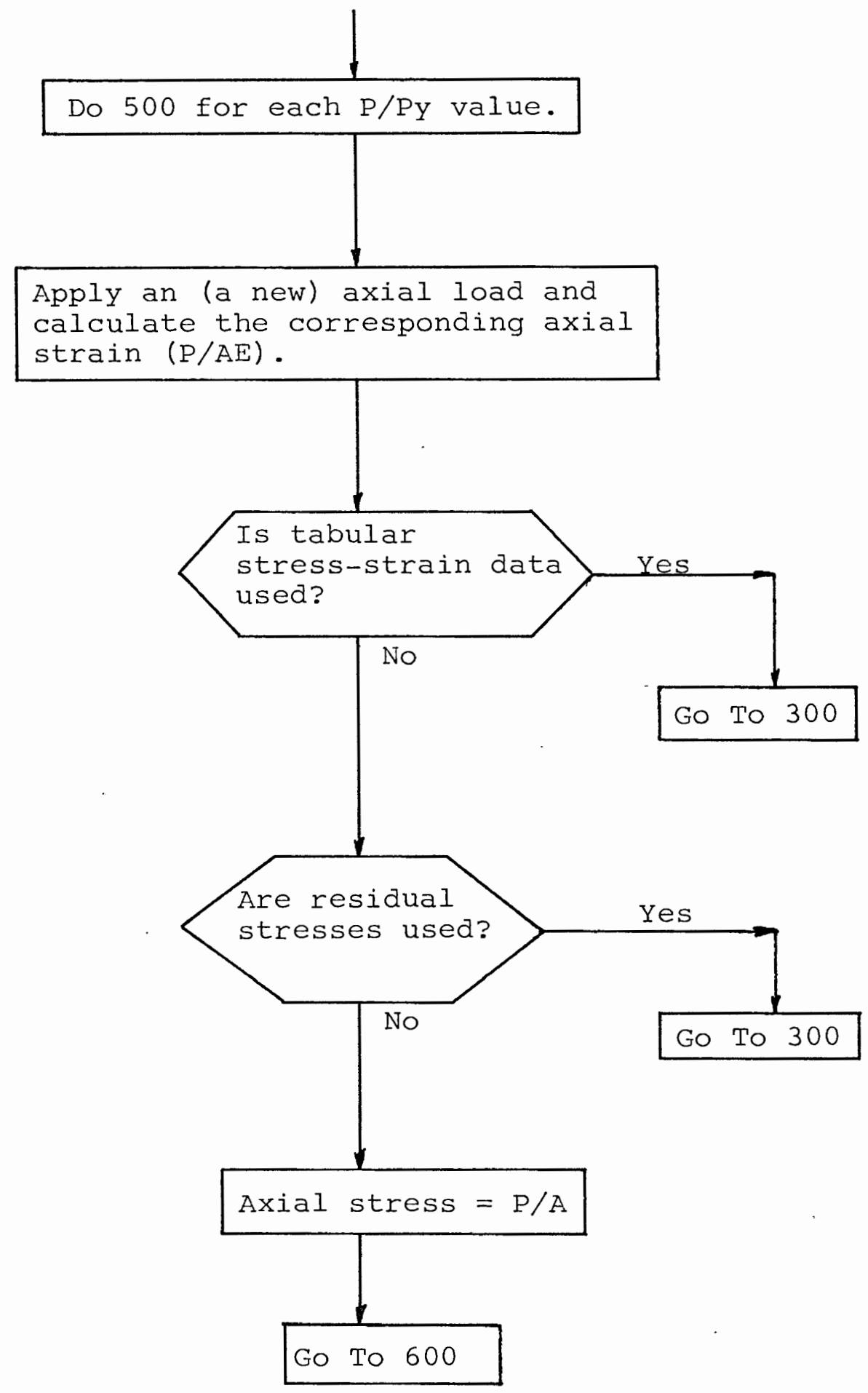




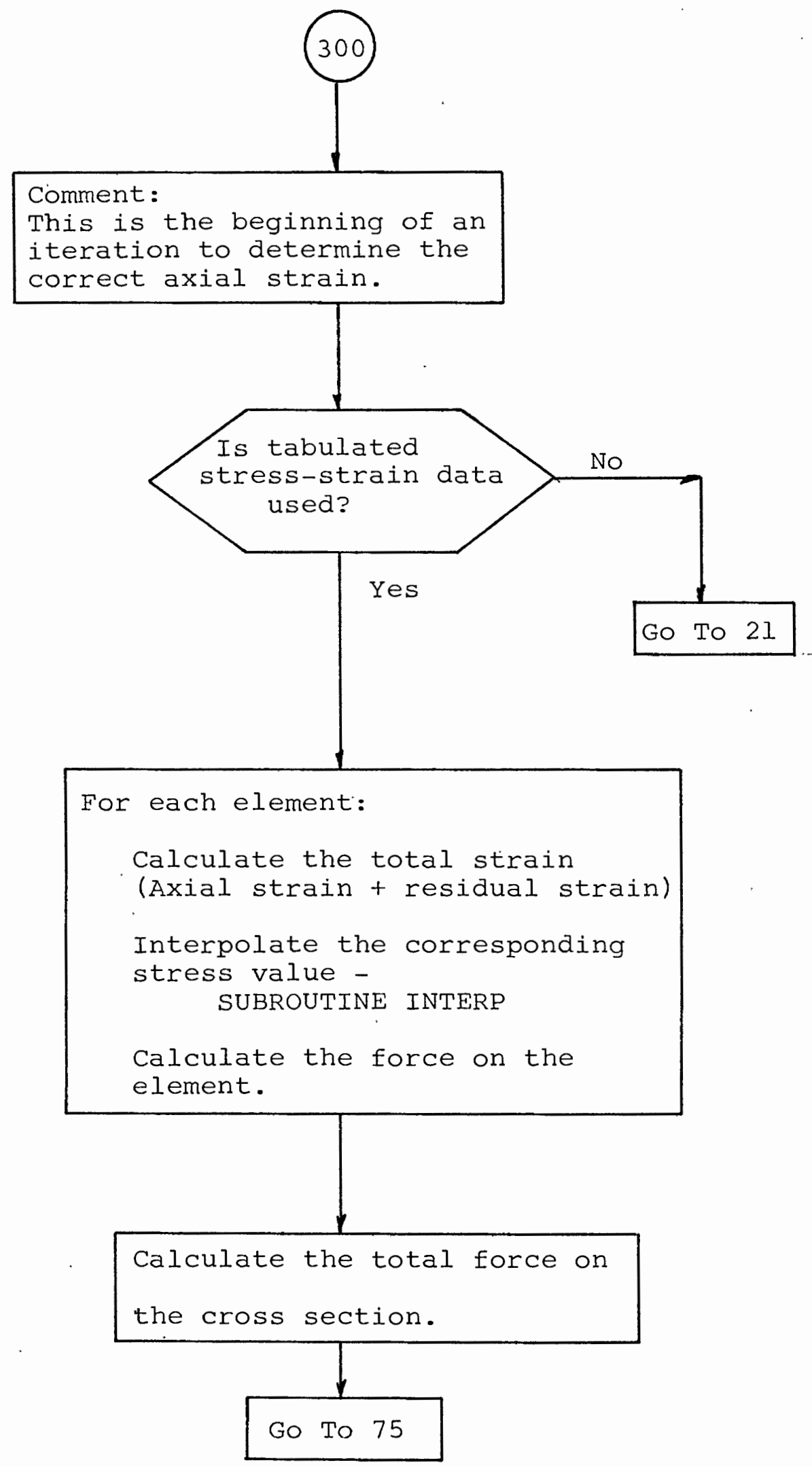




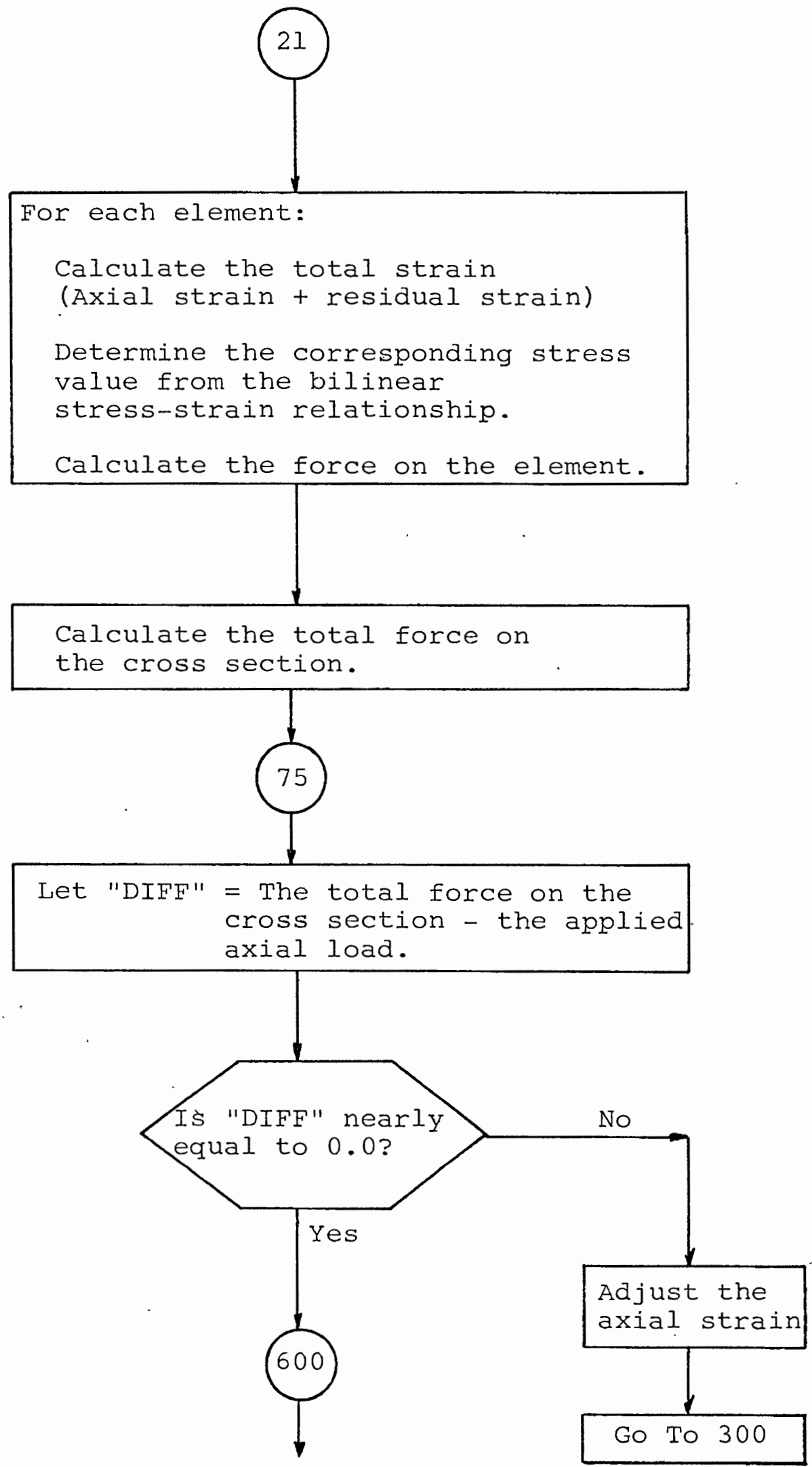




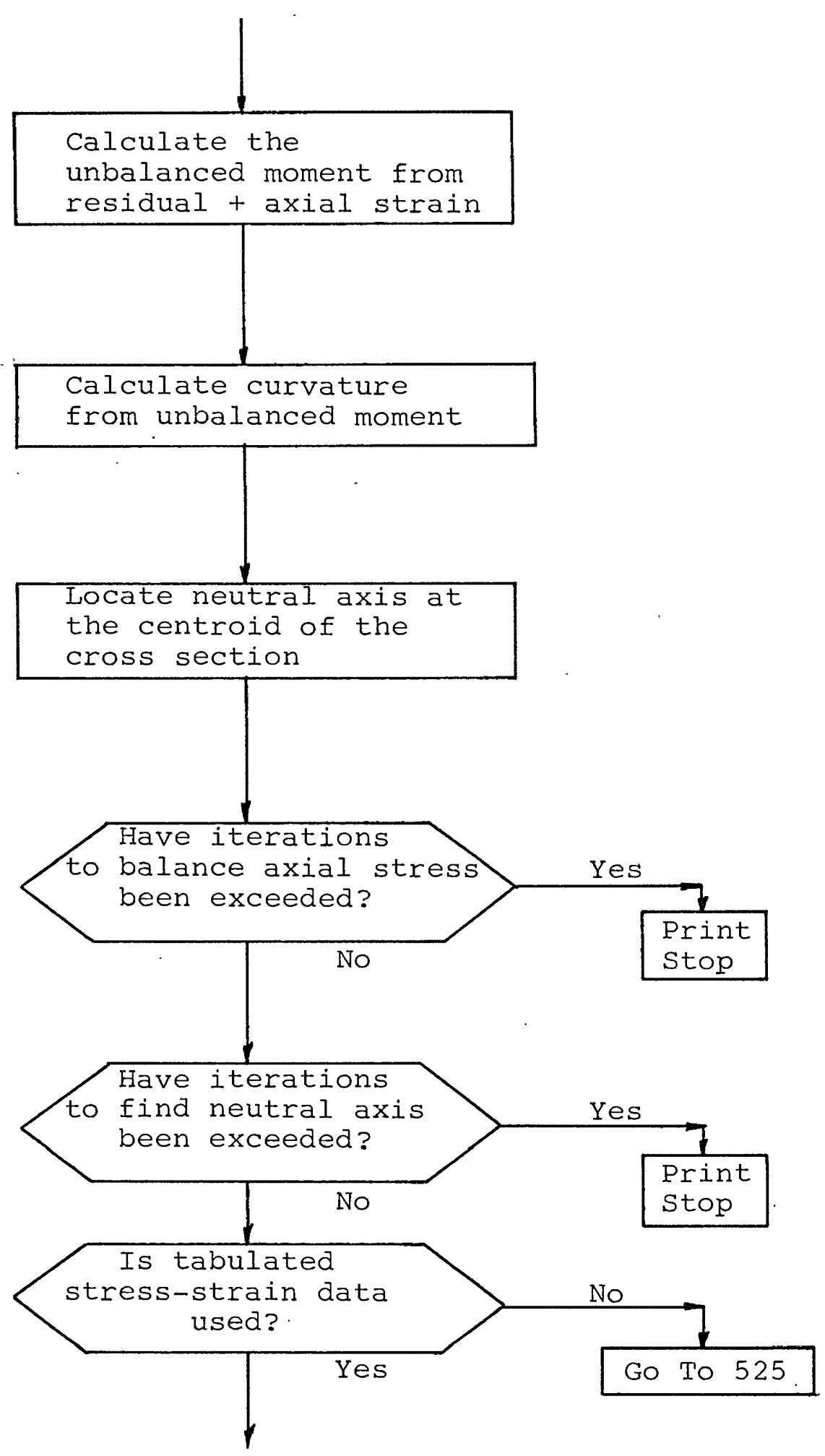




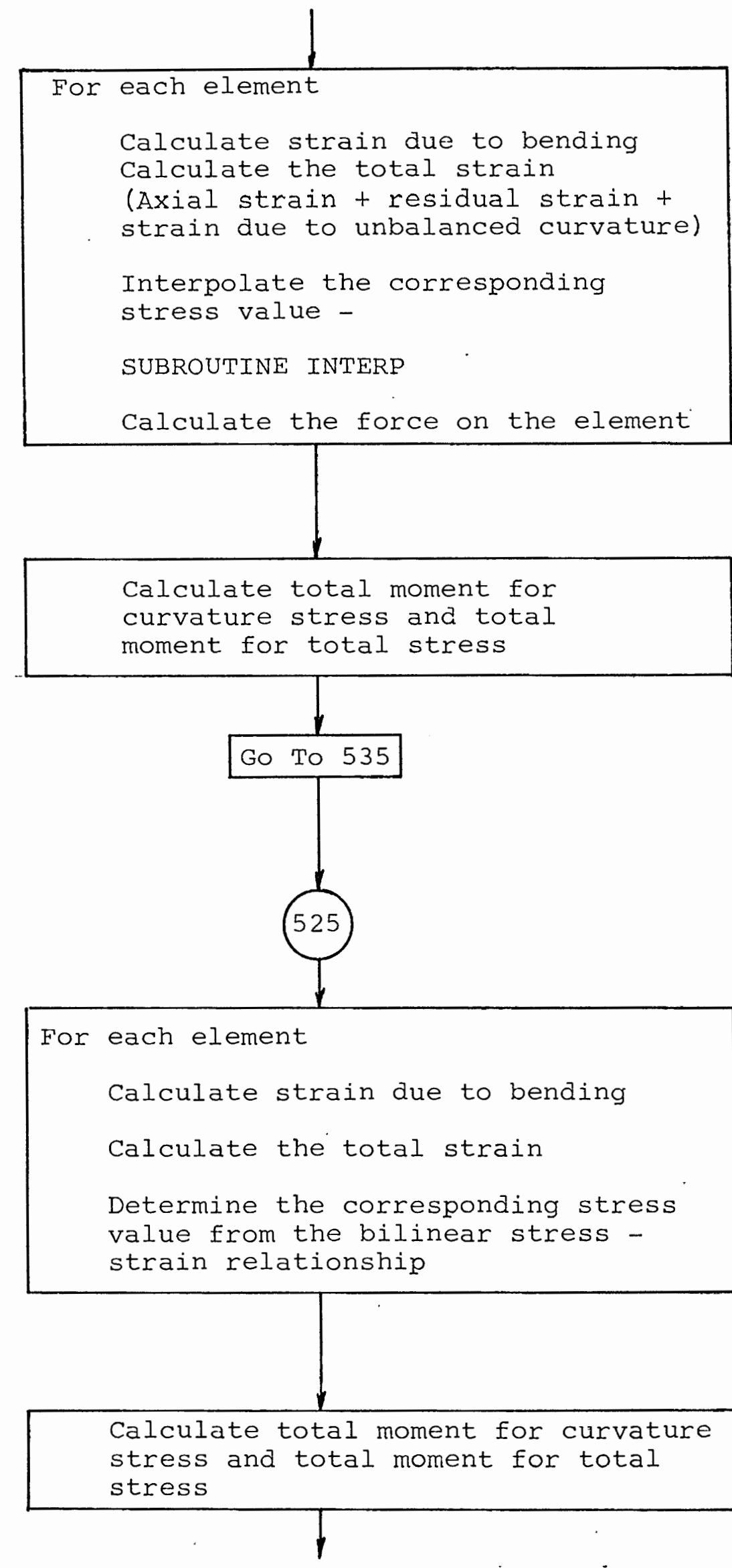



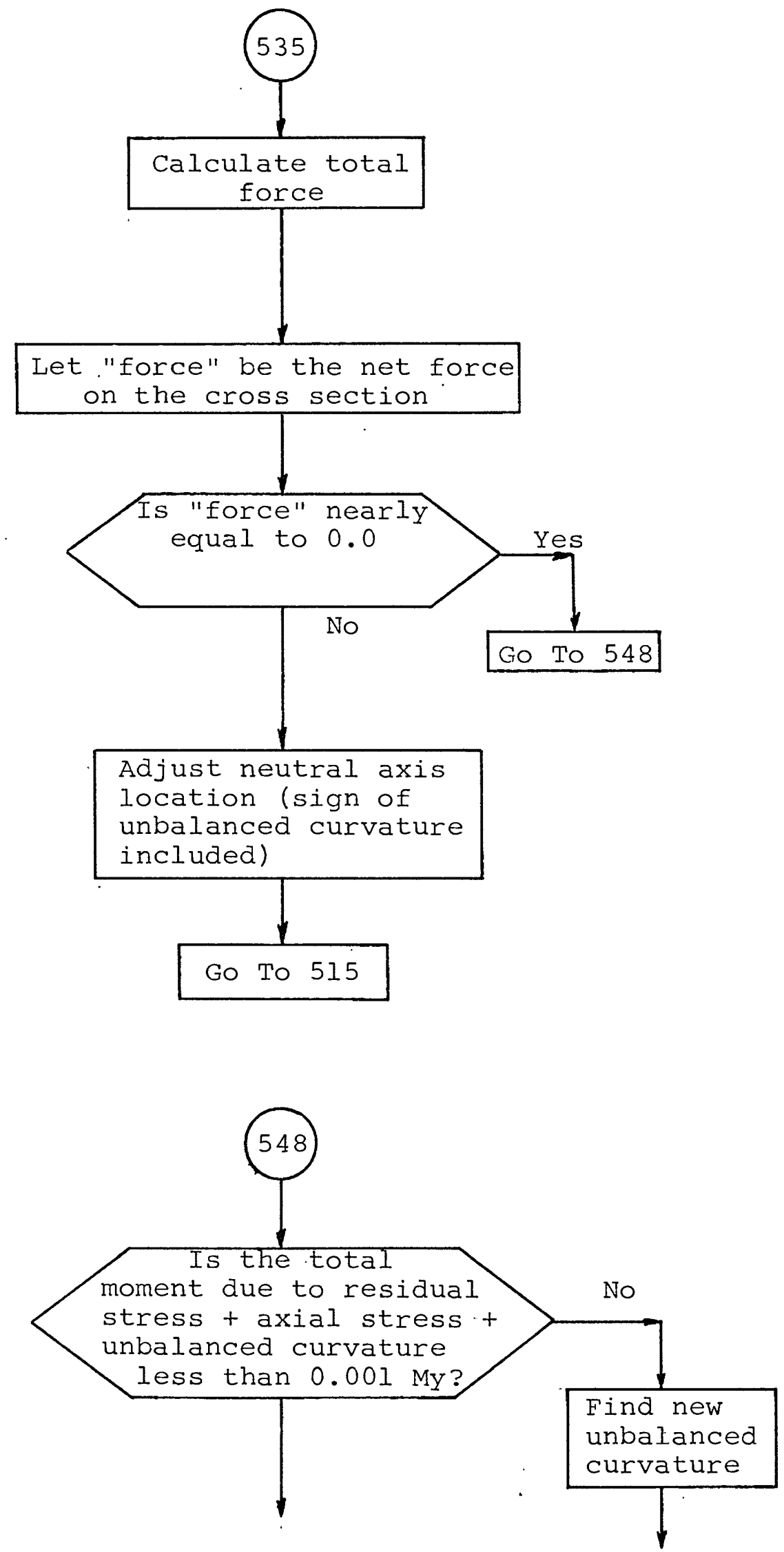


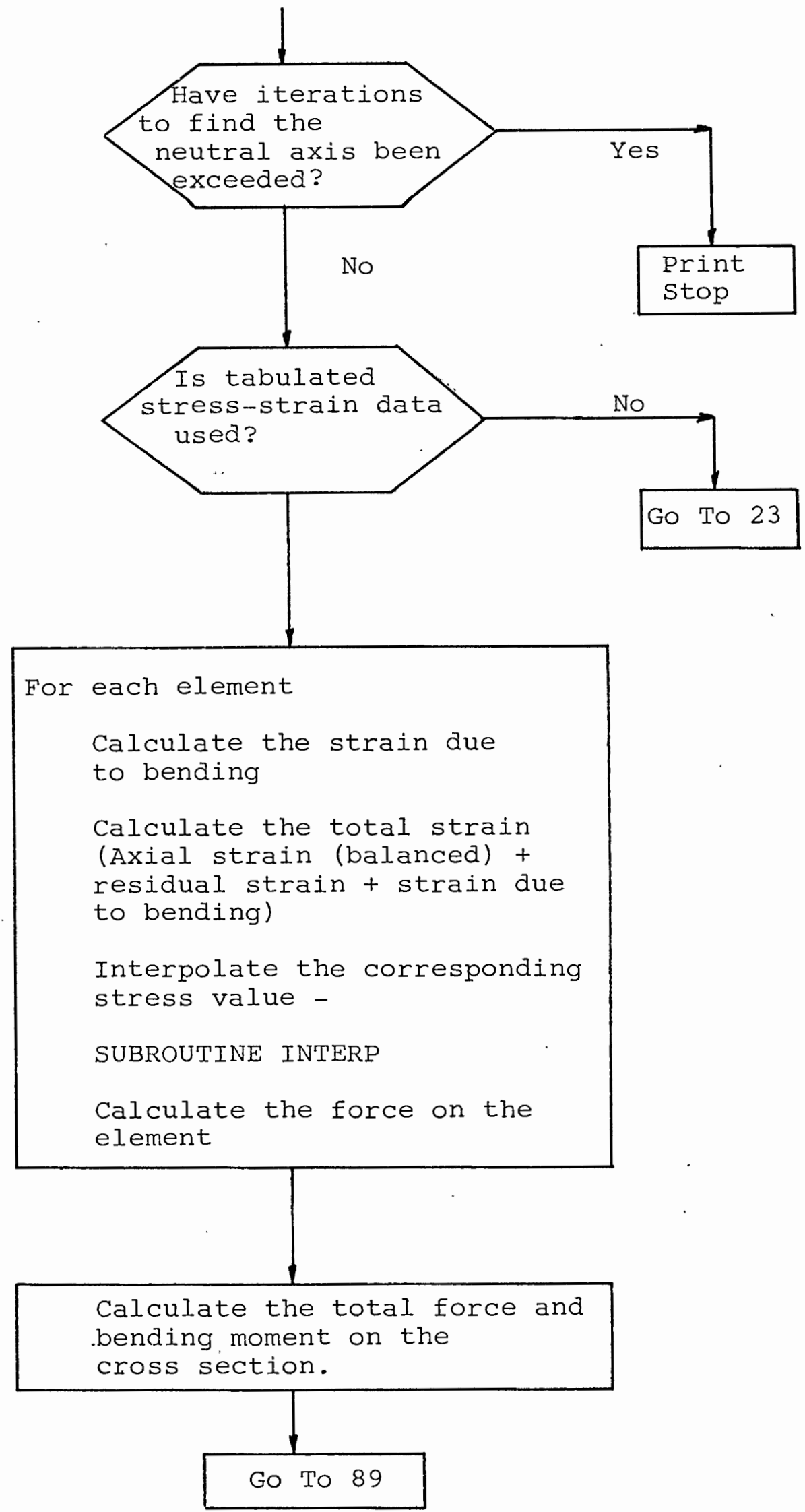




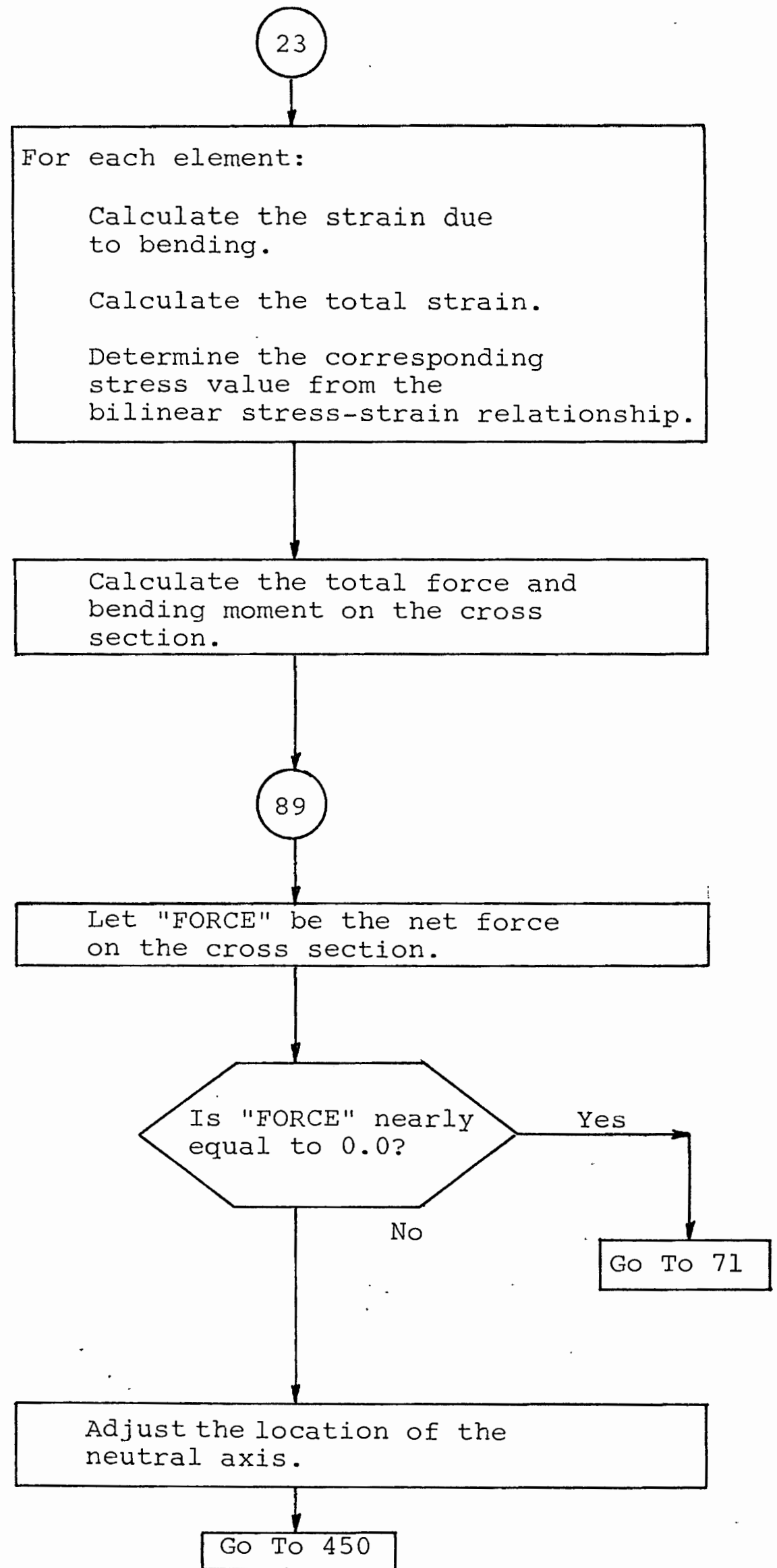




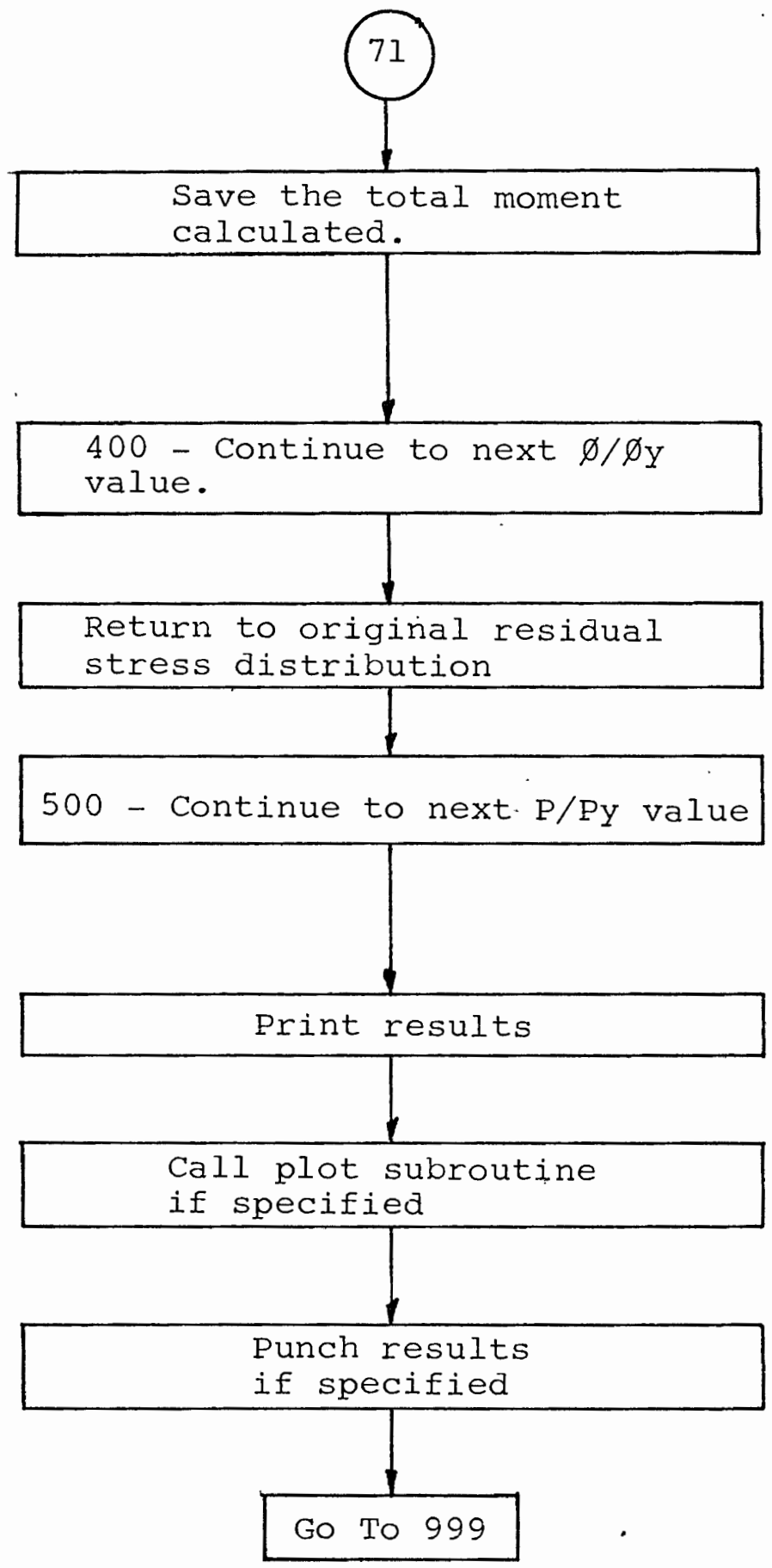


is

$c$

넌:

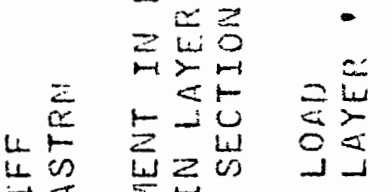

$\overrightarrow{0}$

0

$\mu \geqq$

on a

i.

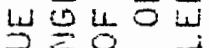

- $\sum^{\circ}=$ 虫

IU

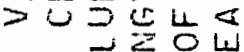

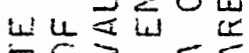

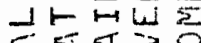

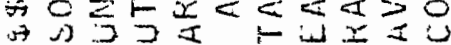

moj, 10 至

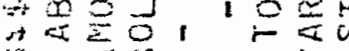

if

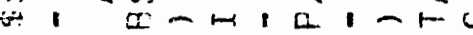

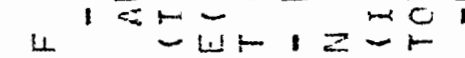

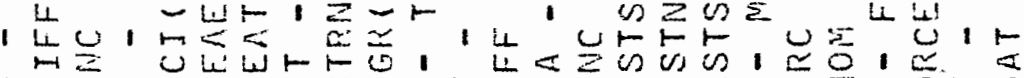

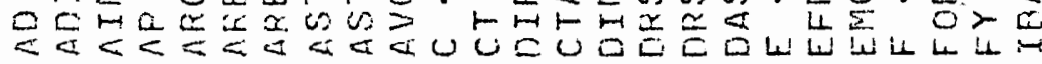

$\rightarrow \underset{2}{2}$

$\Xi$

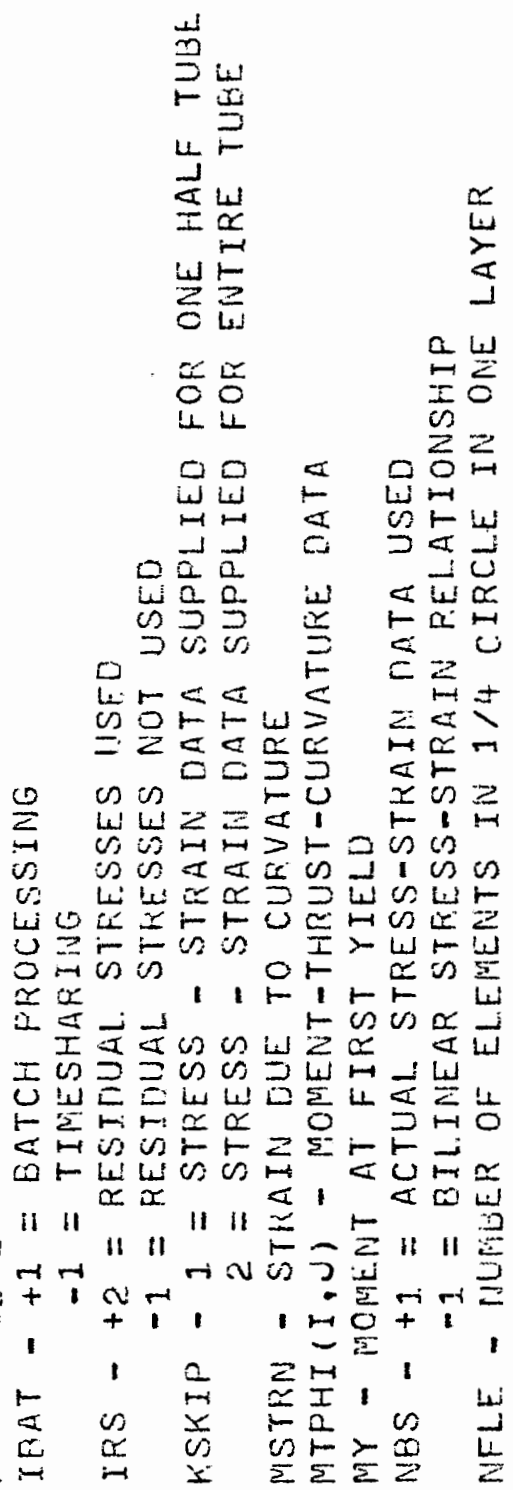

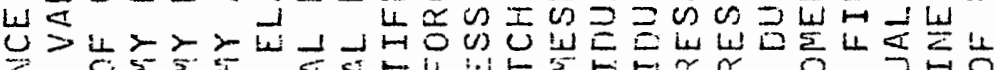

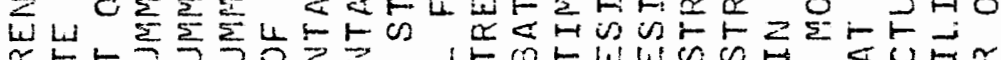

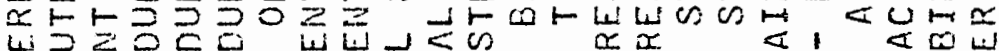

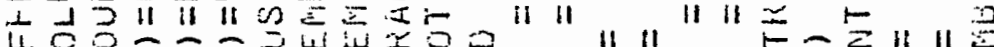

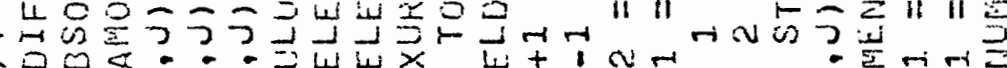

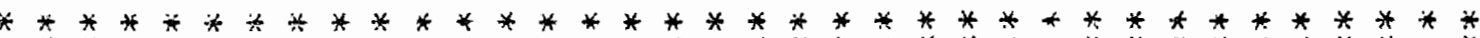

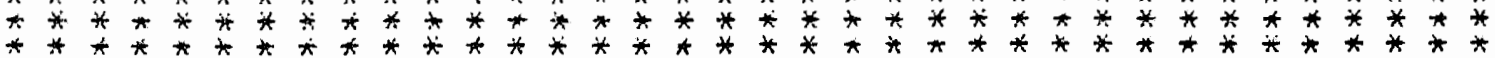

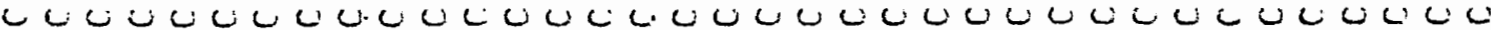


$\frac{x}{4}$

$\stackrel{4}{2}$

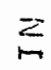

$\underset{U}{U}$

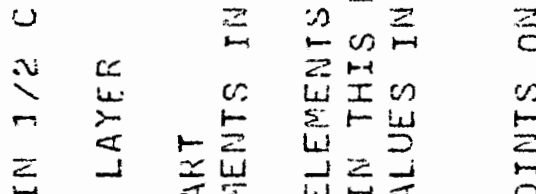

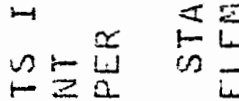

$=\geq$

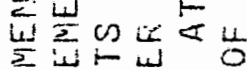

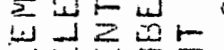
山出官管 $\omega$

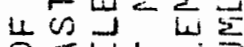
○<山上$4 n>$ a is

$\begin{array}{r}\text { mi } \\ -a \\ \hline\end{array}$ $\infty \equiv$ $\infty \geq \frac{r}{2}$ is $>2$ <i $>$ $-2 \frac{1}{a}$

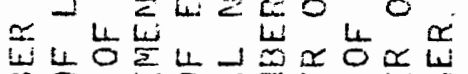

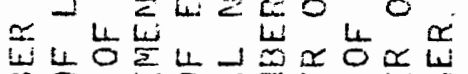

$\cong=$

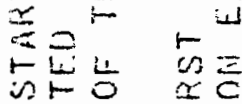
$r \leq x \leq ⿻$ $\varepsilon=x<i<$

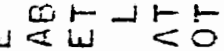

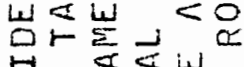
v

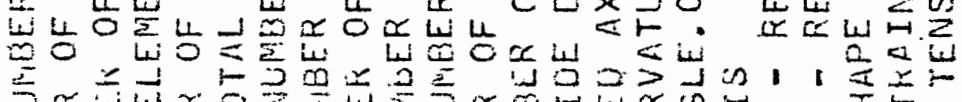

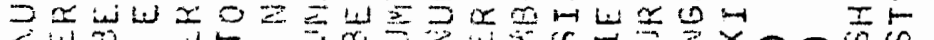

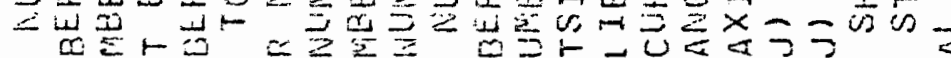

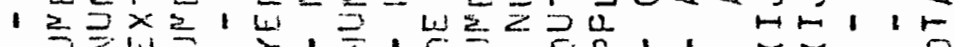

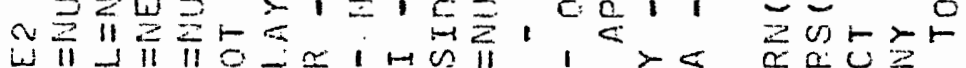

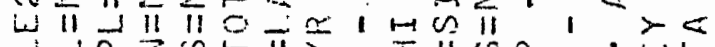

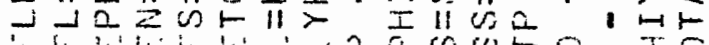

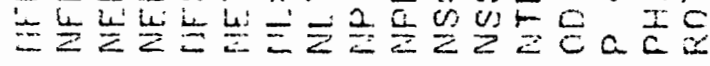
E ELL 단ㄹㄴ

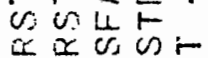

$\frac{1}{6}$
$\frac{\pi}{0}$
0
$\frac{0 x}{a}$

$\cos$

$x$

$\varangle$ L!

لن

ro is

$5>2$

足范

$\rightleftarrows \pm$

岁这

is

जu

$\leftarrow \leftarrow$

$\sum_{i=1}^{\infty}$

\&ए $>$

r $\frac{x}{\infty}$ is

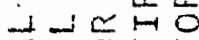
$2 \supseteq$

$\Omega_{n \rightarrow b} \cup$ () V: L $0 \lim _{0} \frac{1}{\mathrm{U}}$ t Cs L L $\sum_{4} \omega u$ $\sum \omega \approx \infty$

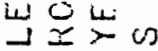

$\vec{u}=0$

- 10

它员它F

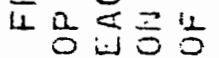

L!

$\omega-4-\omega$

एक

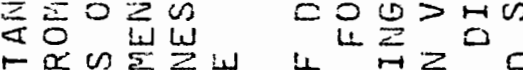

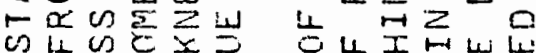

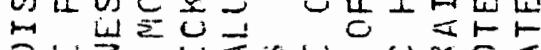
$=\omega \geq \mapsto$ व

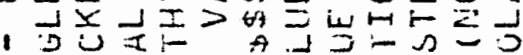

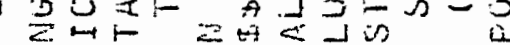

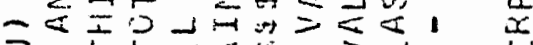

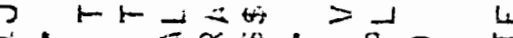

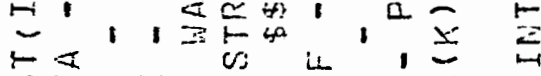
$n+2 \sum 1,140,1$

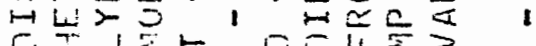

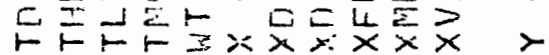




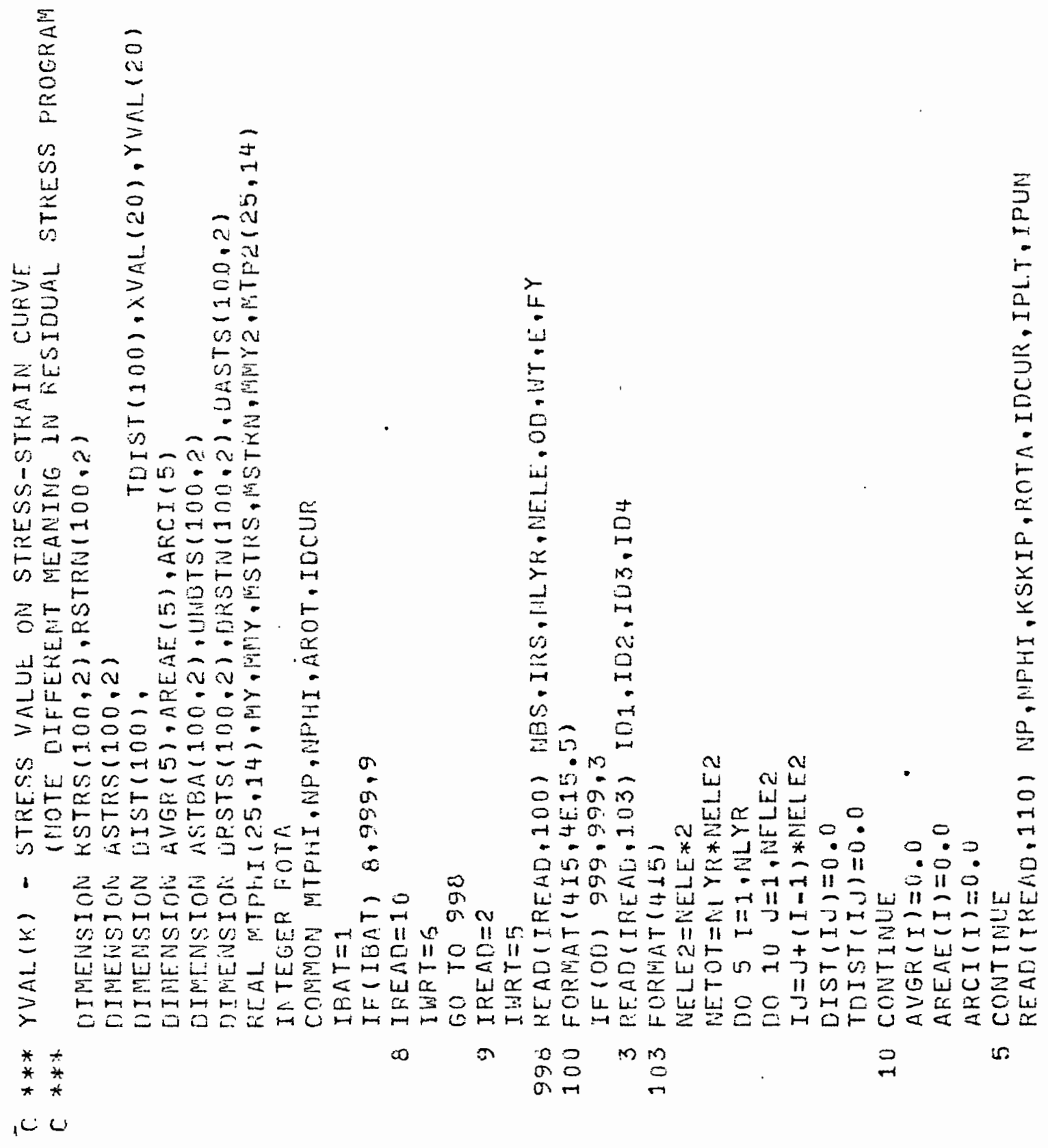

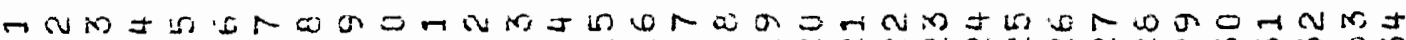

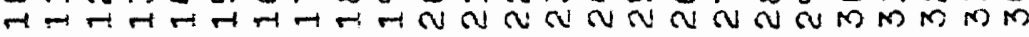




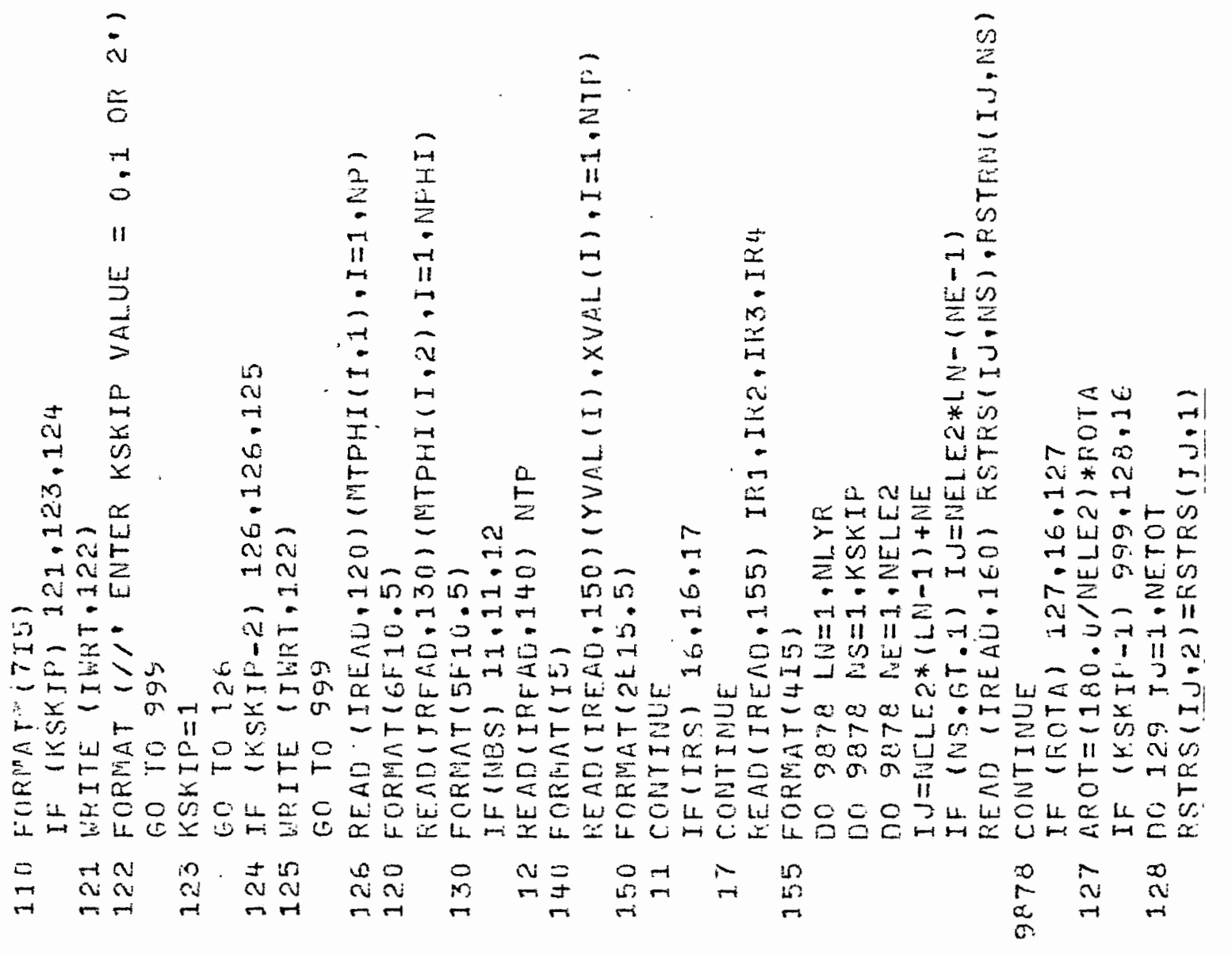

15.

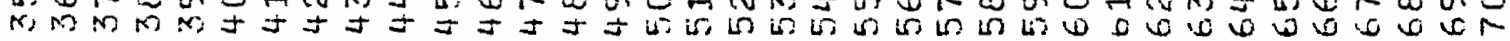




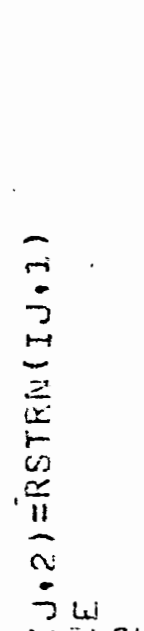

is: $\alpha$

ن

$\stackrel{4}{2}$

12

$>2$

Fin

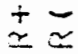

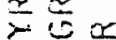

$-\sum>$

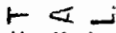

$* * i$

$\rightarrow \infty *$

* $0=$

- $x=$

(1) $>0 \%$

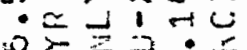

$\rightarrow=0$

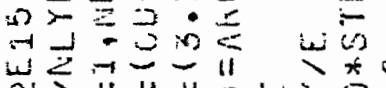

a $a-1111$ - $12>0$.

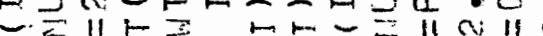

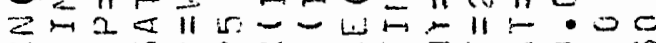

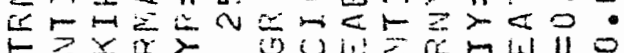

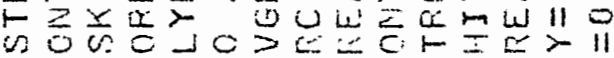

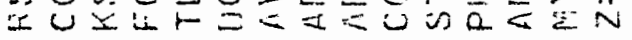

$\begin{array}{llll}0 & 0 & n \\ \pi & 0 & n\end{array}$

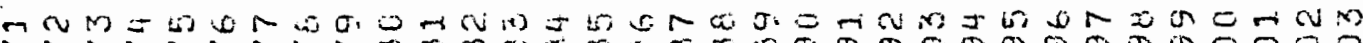
NARTRNAR 


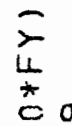

i

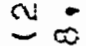

$>\infty$

כ)

인

N山二a c

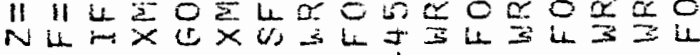

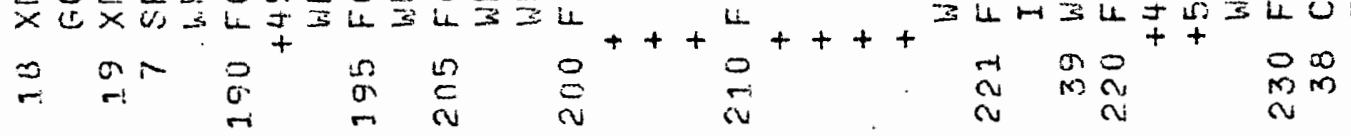

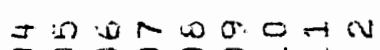

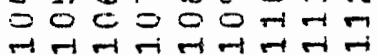

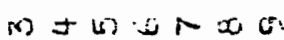
नमनन नमनन नून $\infty_{01}^{-1}$

$\rightarrow N m \pm n$

UN $\mathrm{N} N$

$\mathrm{H}-\mathrm{H}-1$ or 00 N $N$ N 


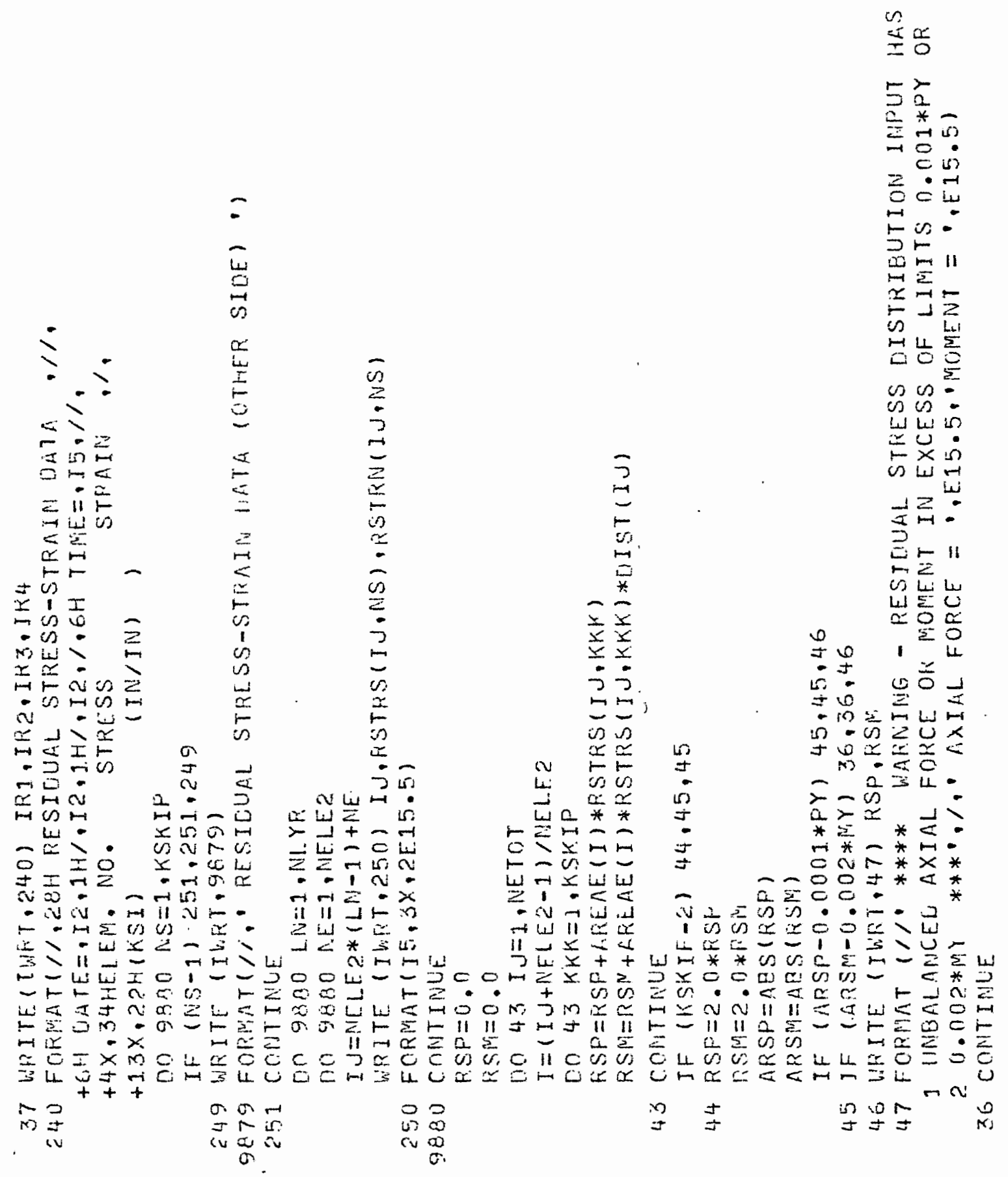




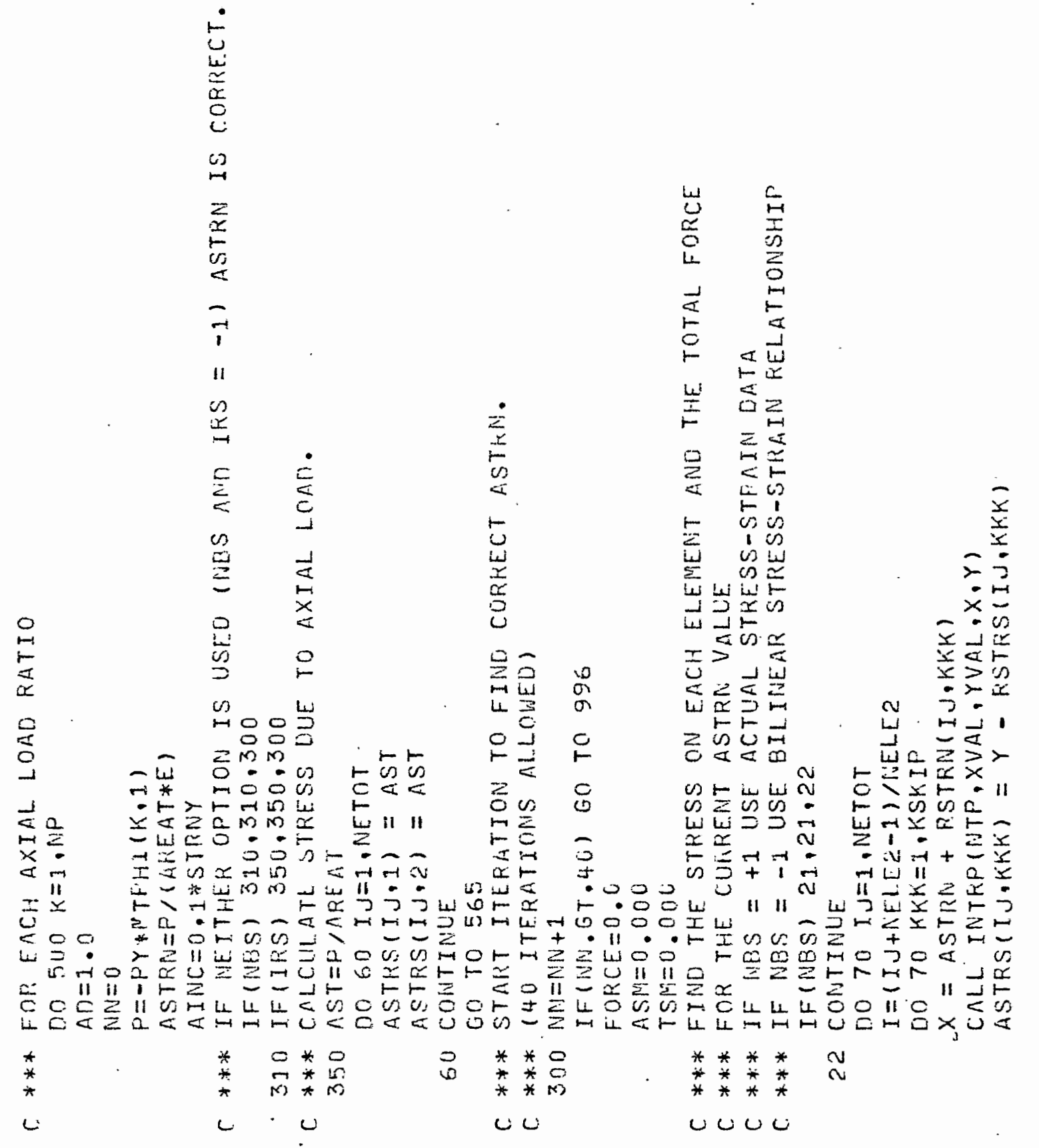




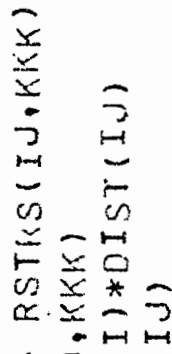

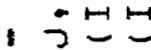
MW $a \times$ is os $x M$

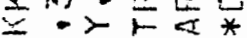
$x$ or $u$ cos $*$ $\therefore N=x-F$ N $\quad-z+x-$ I

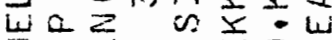
$t a r c-1 x$ $F=\frac{1}{\omega} \bar{x} \div-5$

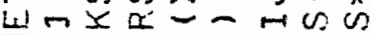
$z 1-\omega x-c x$

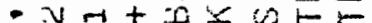
in in

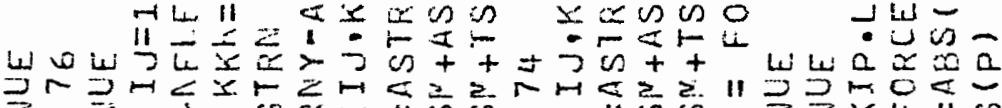

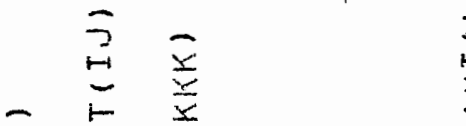

$\overline{2}$

II

is

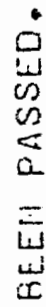

11

is

$\stackrel{0}{0}$

$\stackrel{0}{0}$

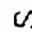

I

0

2
0
5
5
0
0

$\underset{y}{x}$

$y-m$

$\leq x E_{*}-$

$3 x=50$

$-\div-\tilde{F}$

ज)

$\therefore-\frac{1}{4}$

- C L H

$\Leftrightarrow \propto \simeq 0-$

$\approx \sigma_{*} * \cong$

$x \leqslant m$

$+x-\infty$

W-YUW

* $x$ x

$\frac{x}{x} \div \frac{1}{x}$

II $\div-\frac{1}{2}+$

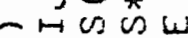

$x-\approx a u$

$x$ s

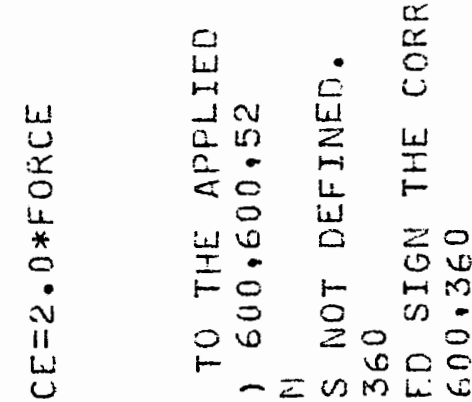

- EnmLE

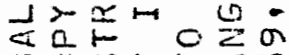

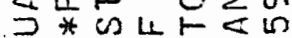

0

U - 4 uO 0300 L 24 Li $c=x$ us $\underset{x}{0}-\hat{0} \leq$ I L

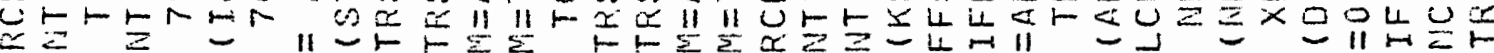

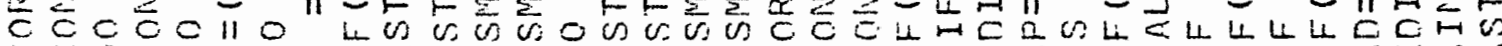

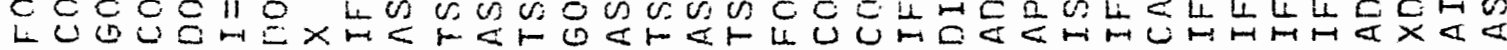

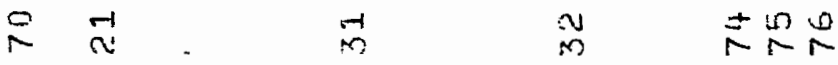

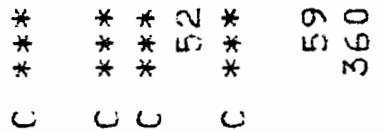

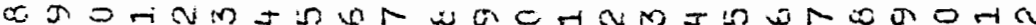

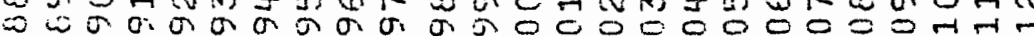

$m$

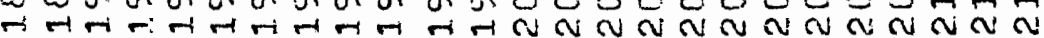
$\underset{4}{ \pm}$

is $w \wedge$ a N $\vec{N}$ त 


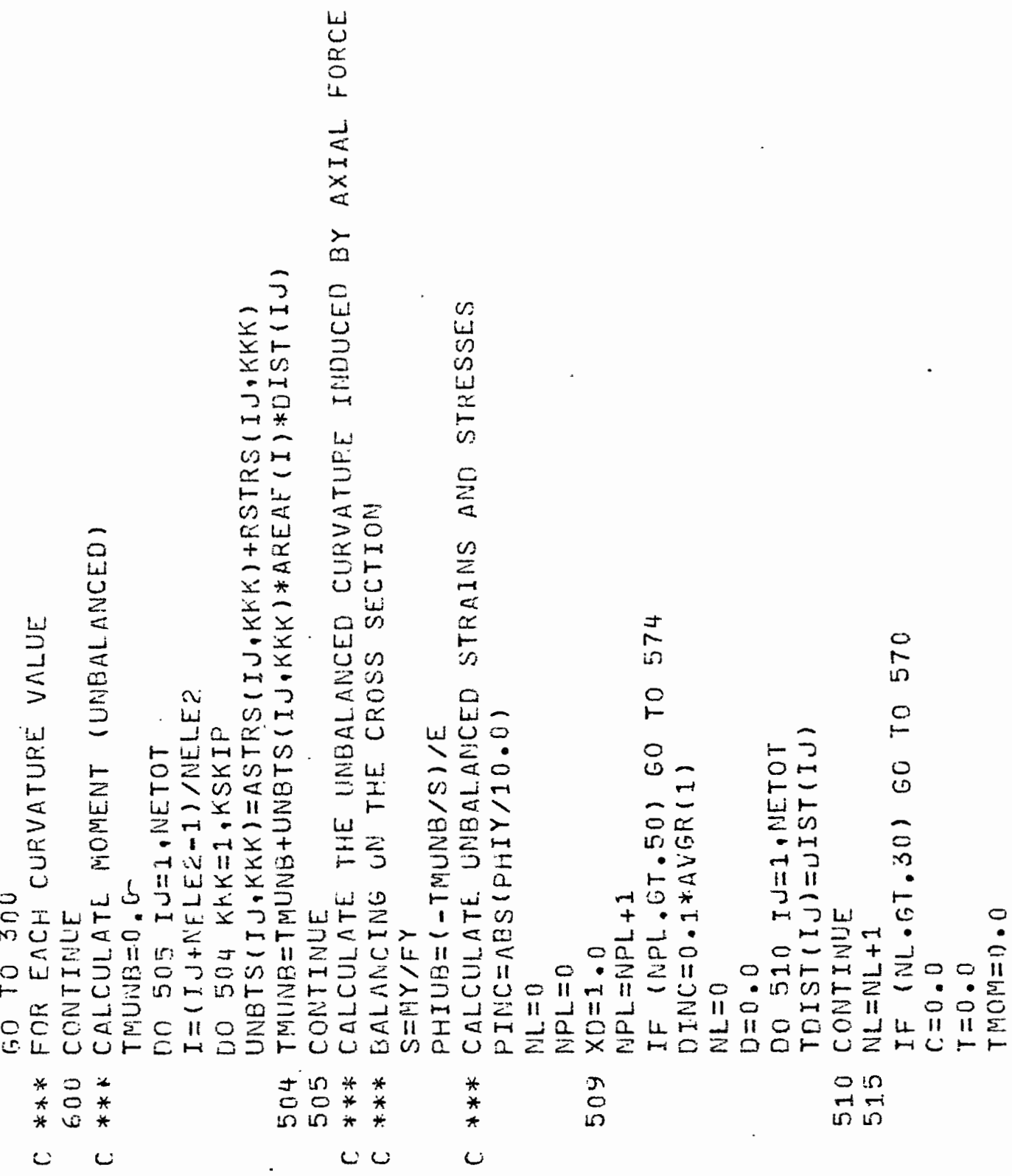

G

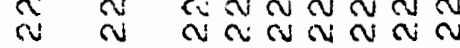

ก $\cos 20$ N
MNM $M$ N

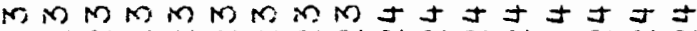
N N N N N N N N N N N 


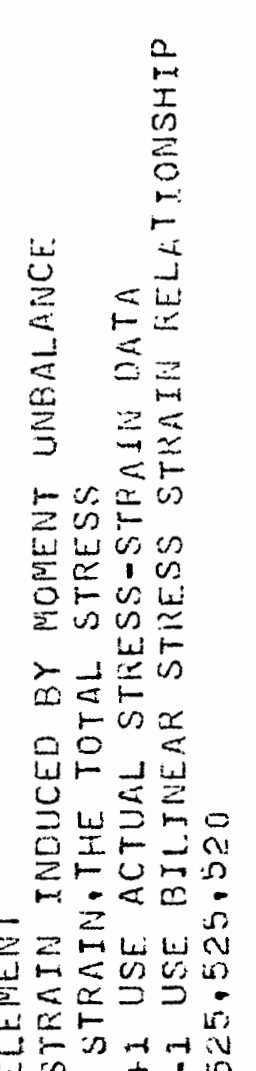

$c^{\omega \infty}+1$ in

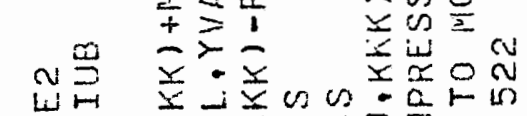

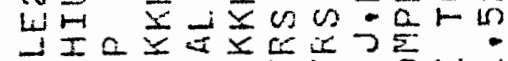

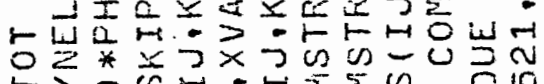

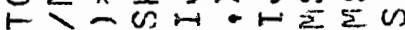

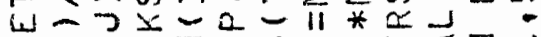

¿

-

il

II
足

vi

$\stackrel{\leftarrow}{\sim}$

L.

卡

$z$

$\underset{\pi}{\alpha}$

$\underset{1}{\leftarrow}$

$\stackrel{4}{5}$

5

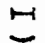

to

要

כ音

$\mapsto-$

$F \stackrel{i}{<}$

绎

$\leftrightarrow 2$

운

**艹心

统

$\approx$

L.

$+$ 11

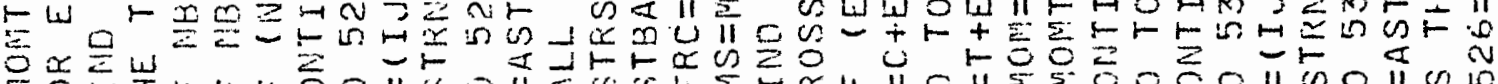

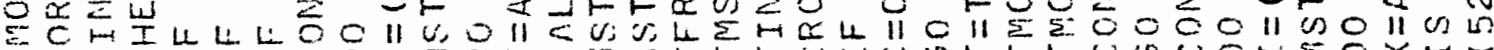

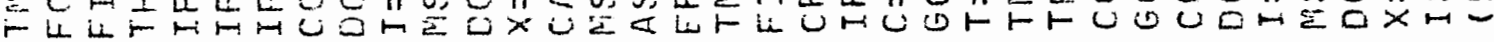

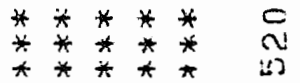

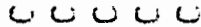

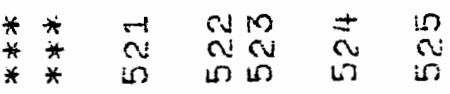

ᄂ U

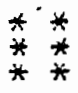

$\cup \cup$

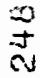

a

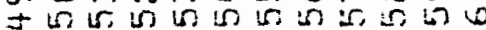

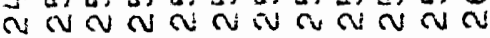

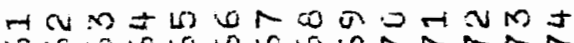

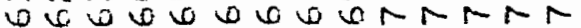
N G N N N N N N N N W N 


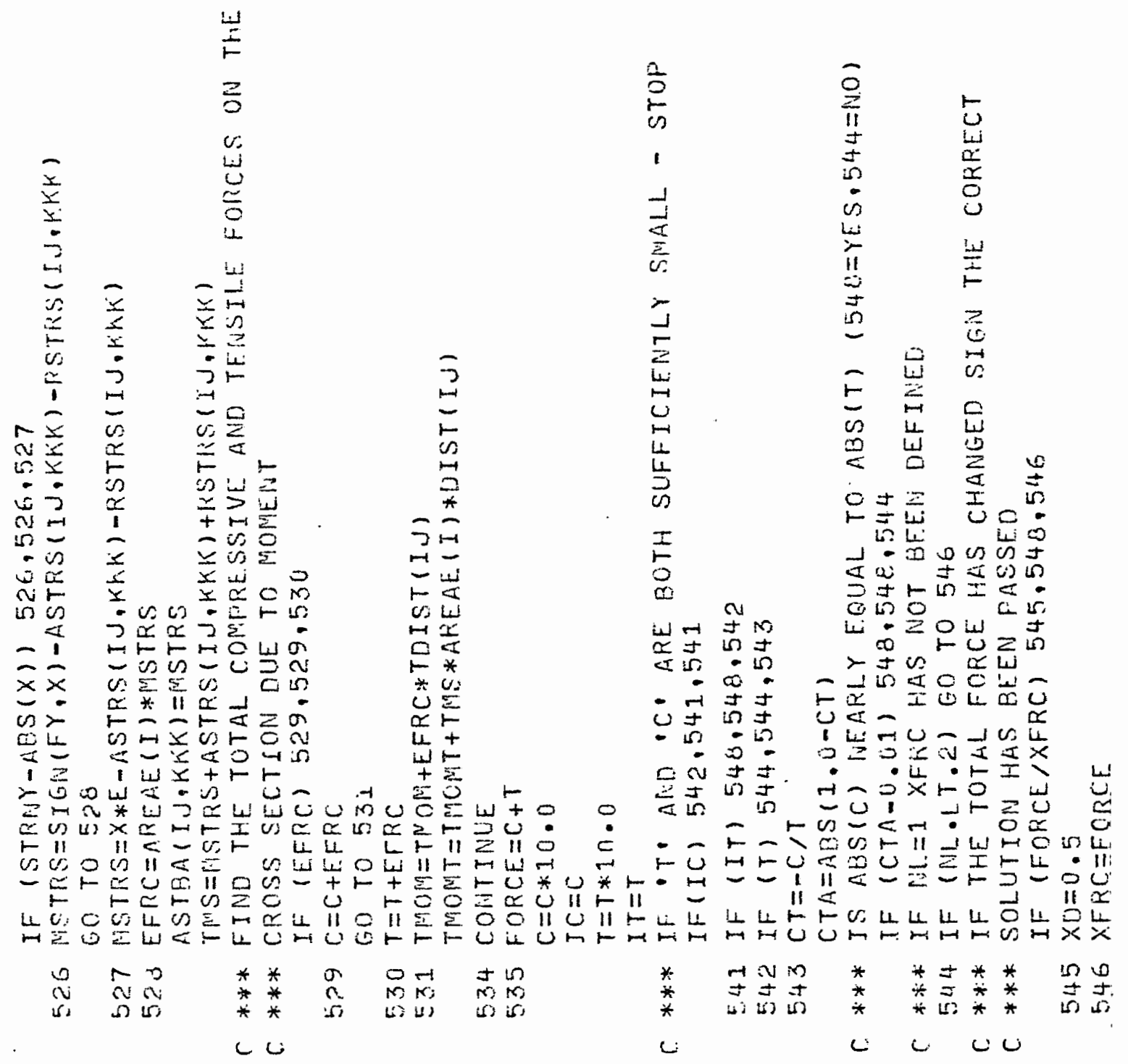

is)

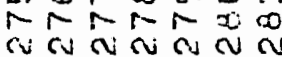

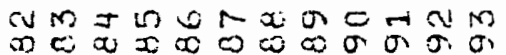

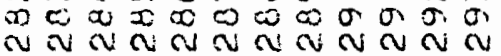

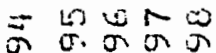

$n$
0
$N$

$\stackrel{0}{0}$ ris $\mathrm{r}_{3}$

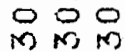


$=$
0
0
0

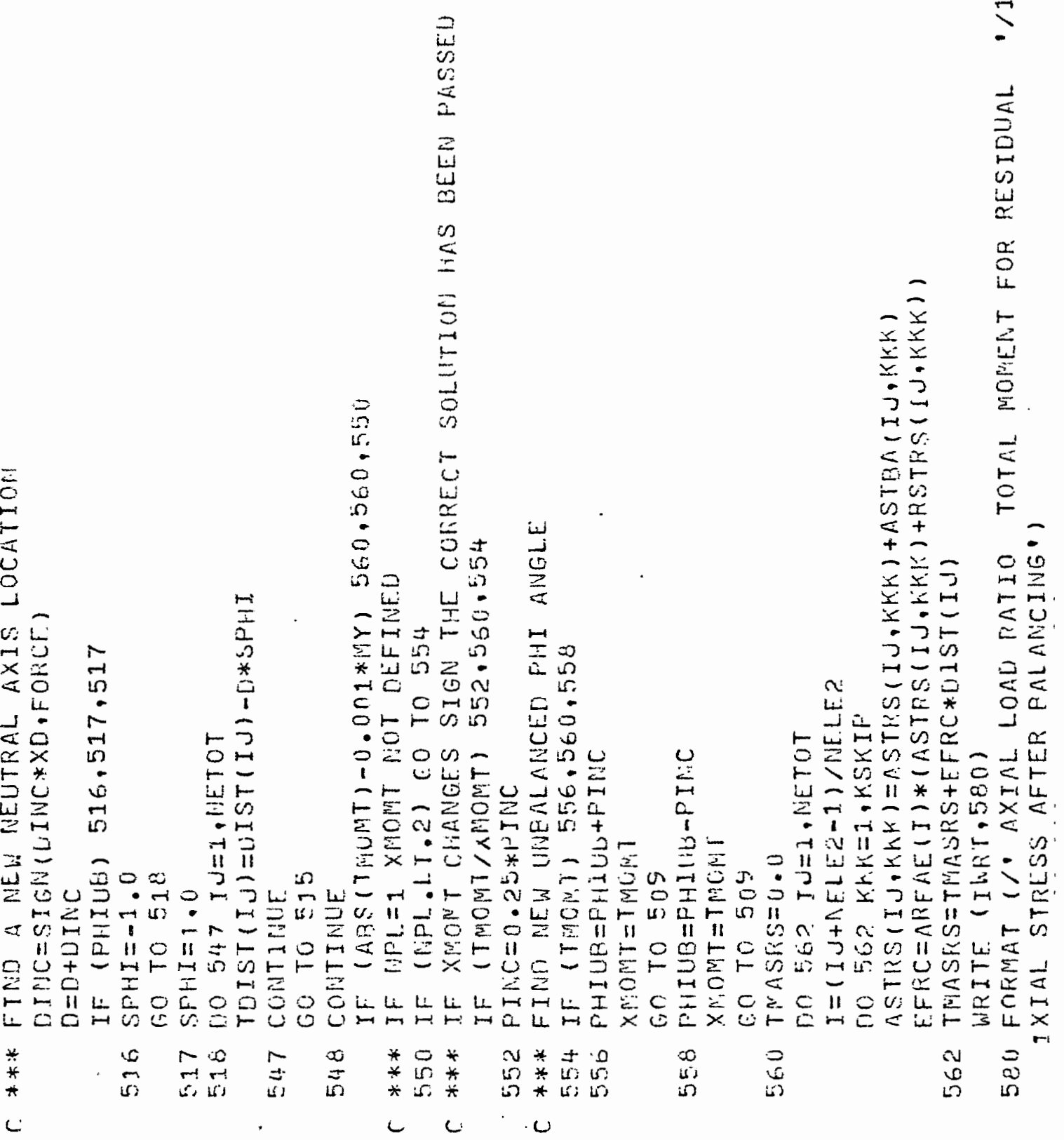

J

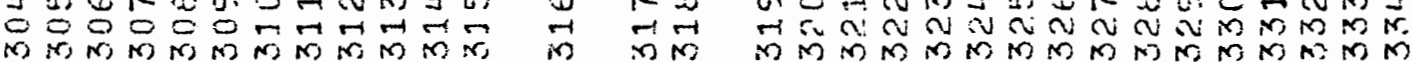




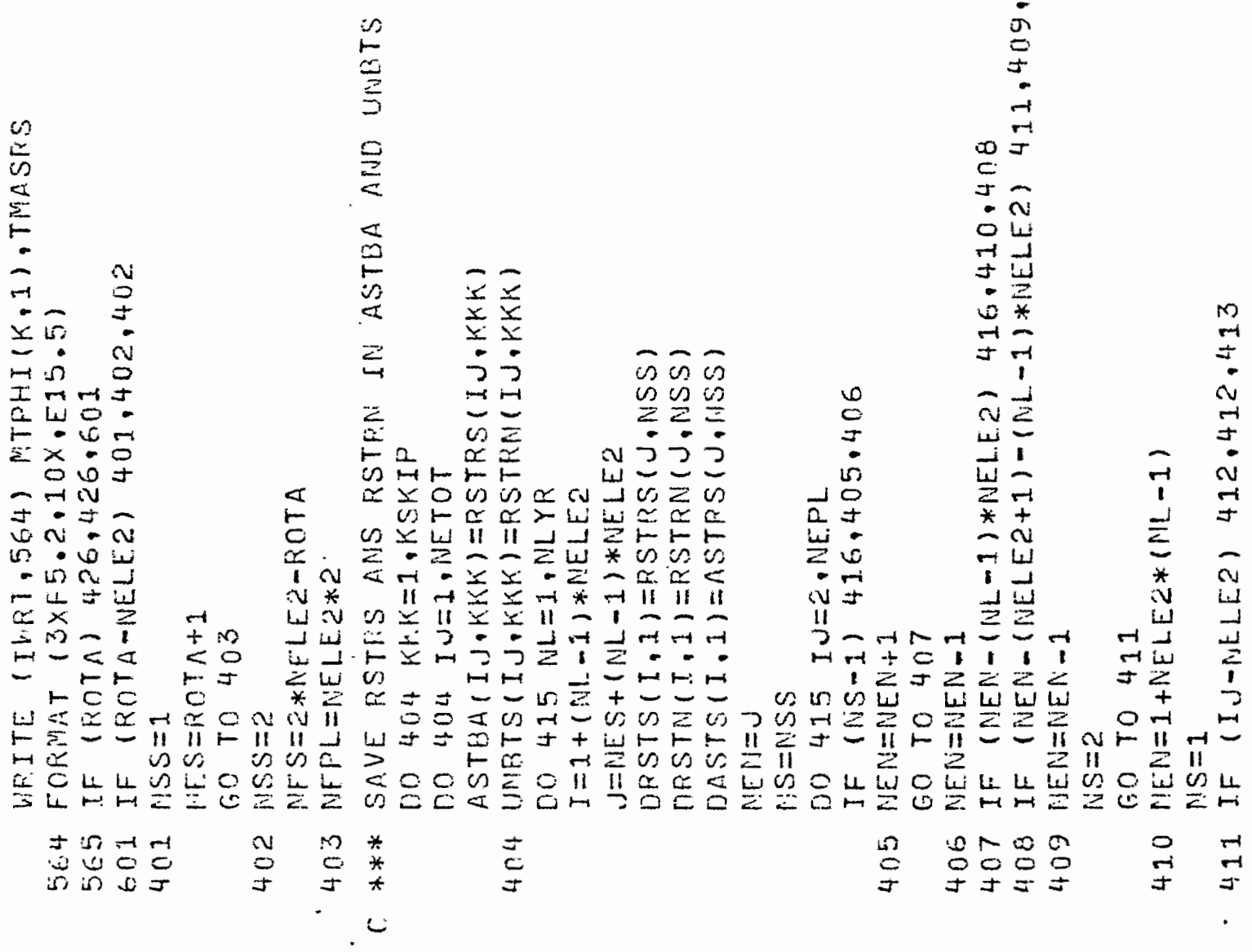




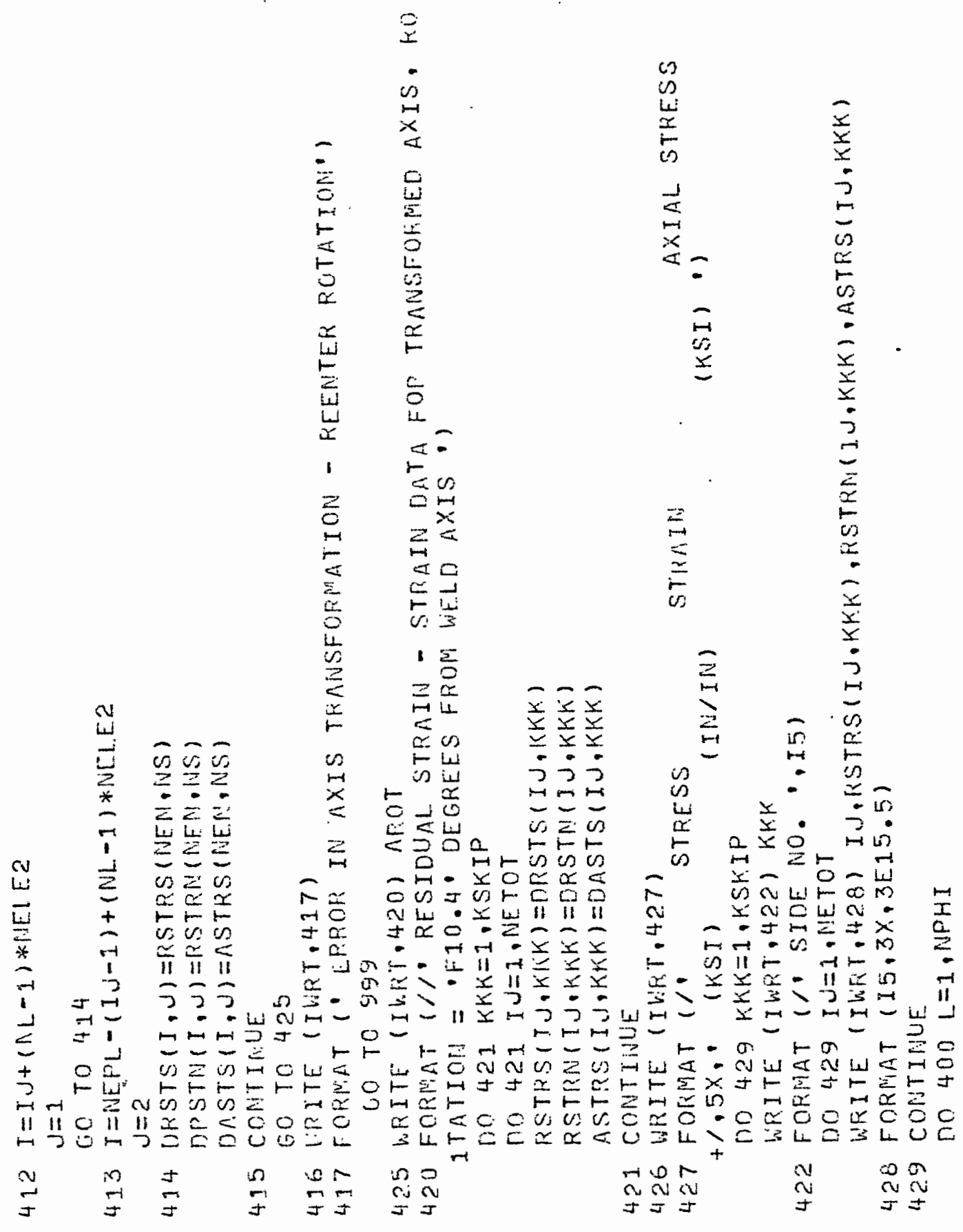

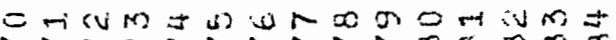

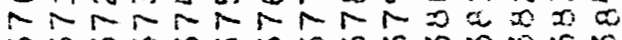

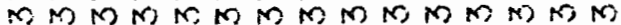

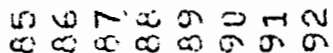
$M M M M M M M$ $n \neq$ in

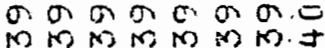




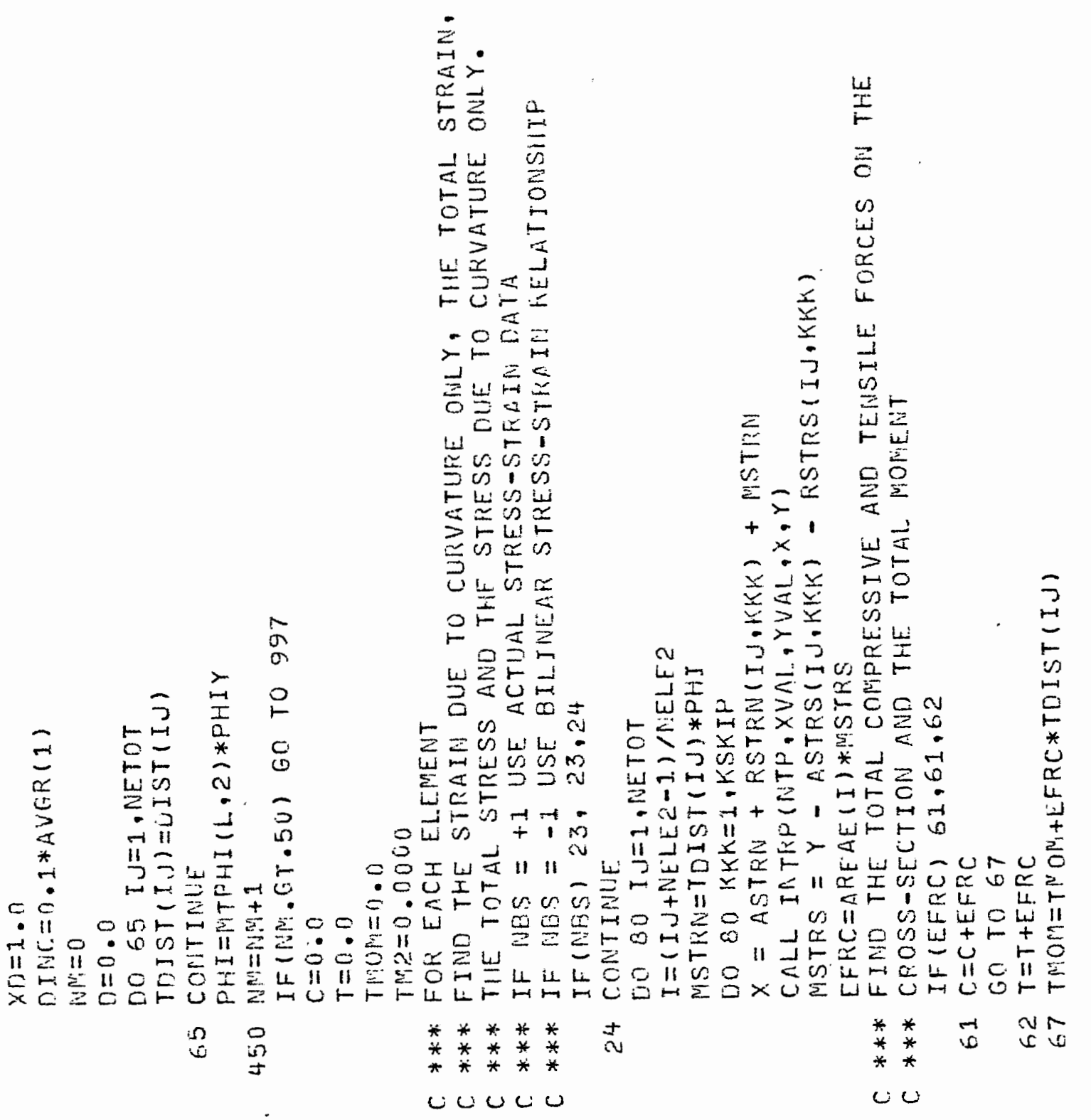

m N

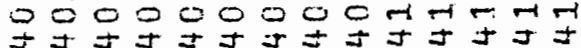

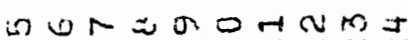

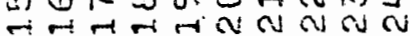
$\exists \pm= \pm \pm \pm J \pm \pm=$

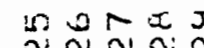
N $N$ N I $千$ I 


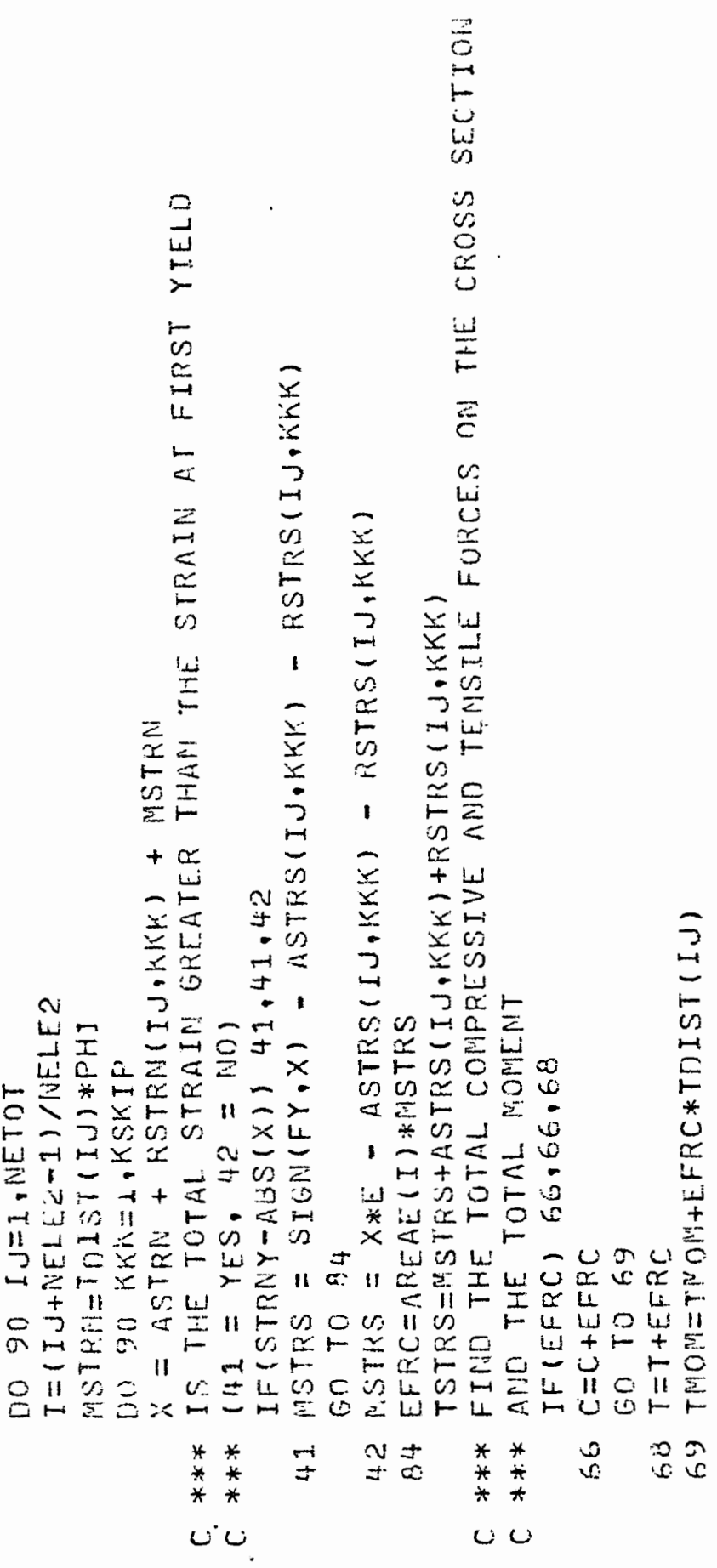

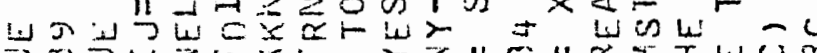

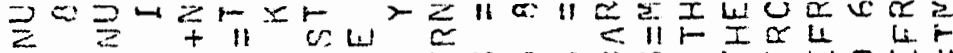

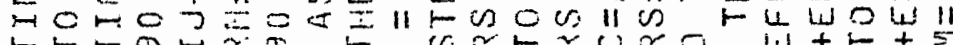

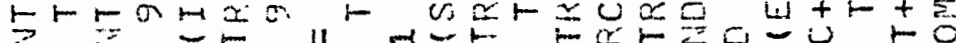
C

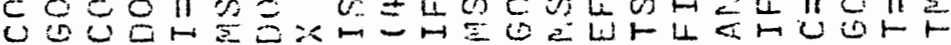
$\vec{a} \quad N$

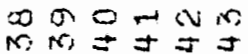

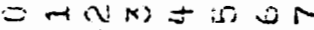

\pm m
+ \pm \pm
$+ \pm \pm \pm J$

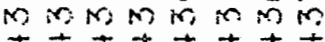

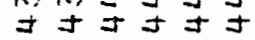



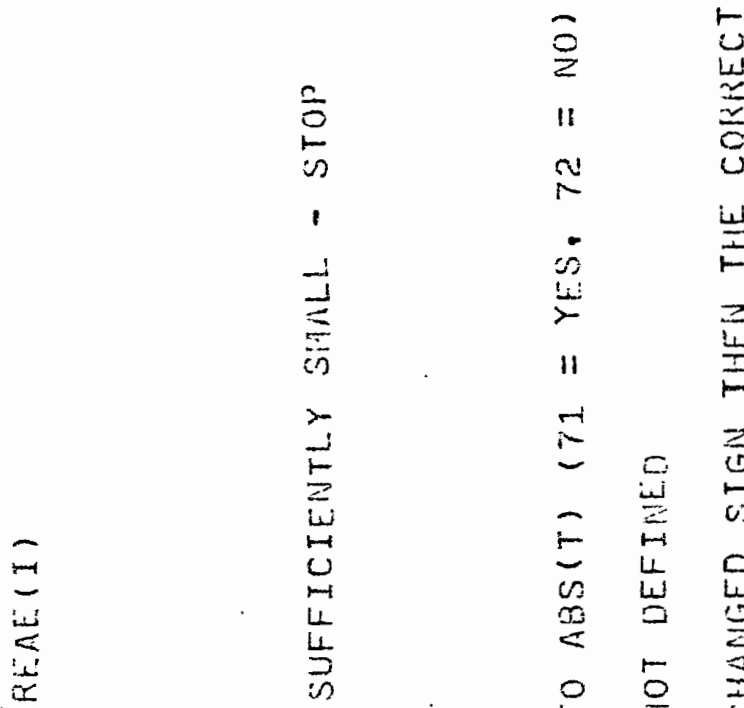

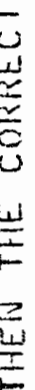

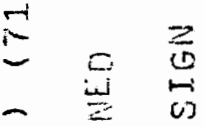

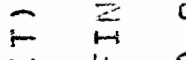

ज L ए

$\underset{0}{\infty}$

$0 \quad 5$

in

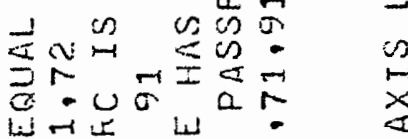

$\frac{1}{\alpha}$

i $\frac{1}{x} 00=\pi$

Nur $-\pi$ ou

- or ar

$-\alpha-i$

oriv

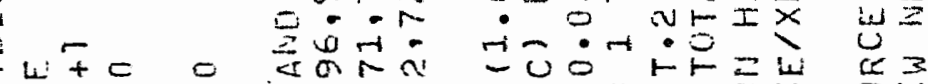

$1 \mathrm{Li}-\underline{L}$

$\frac{1}{2} \frac{2}{1} \frac{\alpha}{1}$

- 0 ov

w

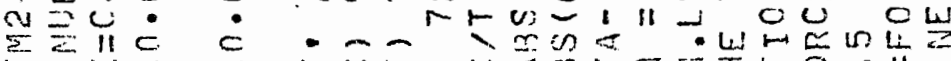
他

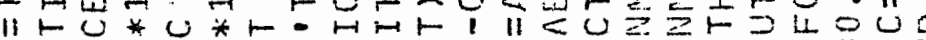

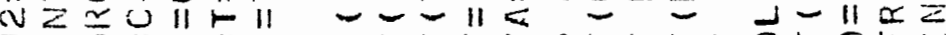
EOC

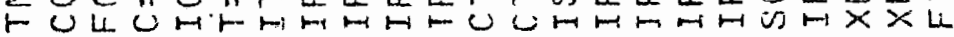
$\sigma_{0} \sigma$
*

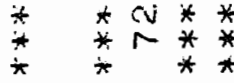
त)
⿶

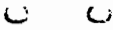
ic

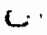

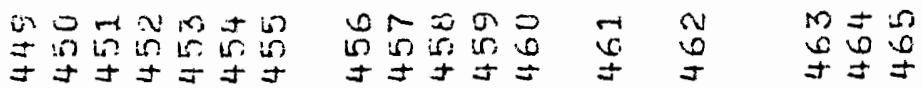




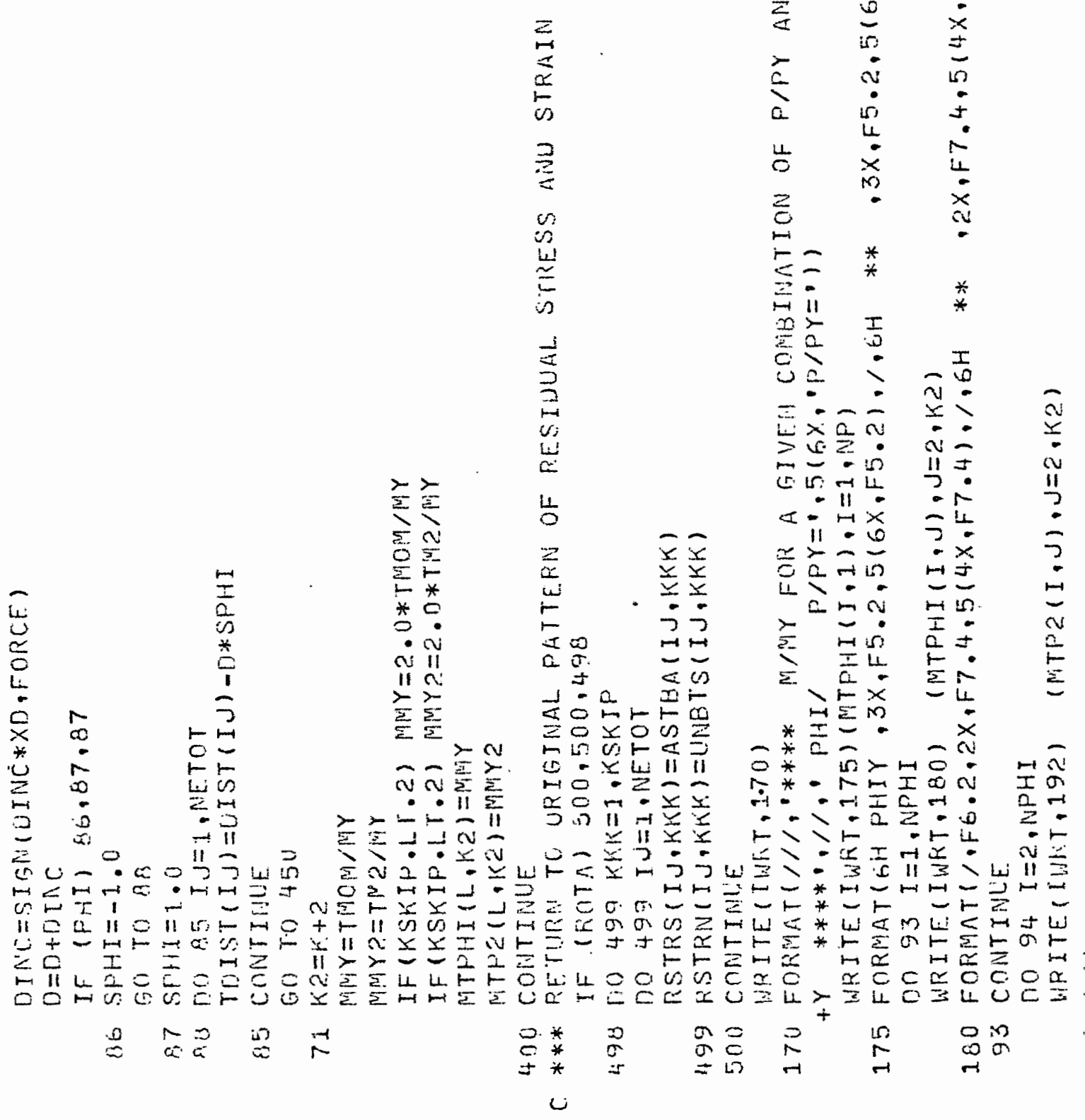


2

$\div 0$

$x+2 \div \infty$

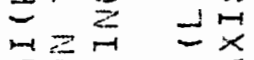

I $20 \quad \mapsto<$ EF

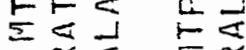

сभH W W

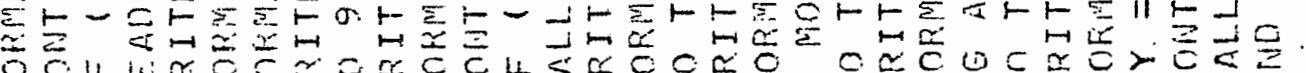
бо山 L

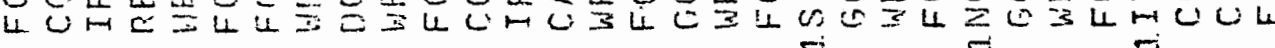

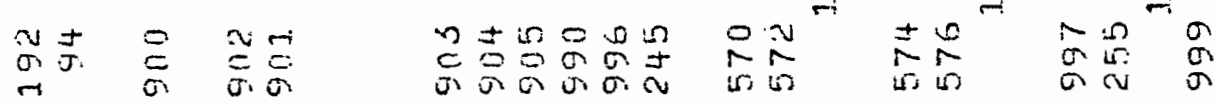




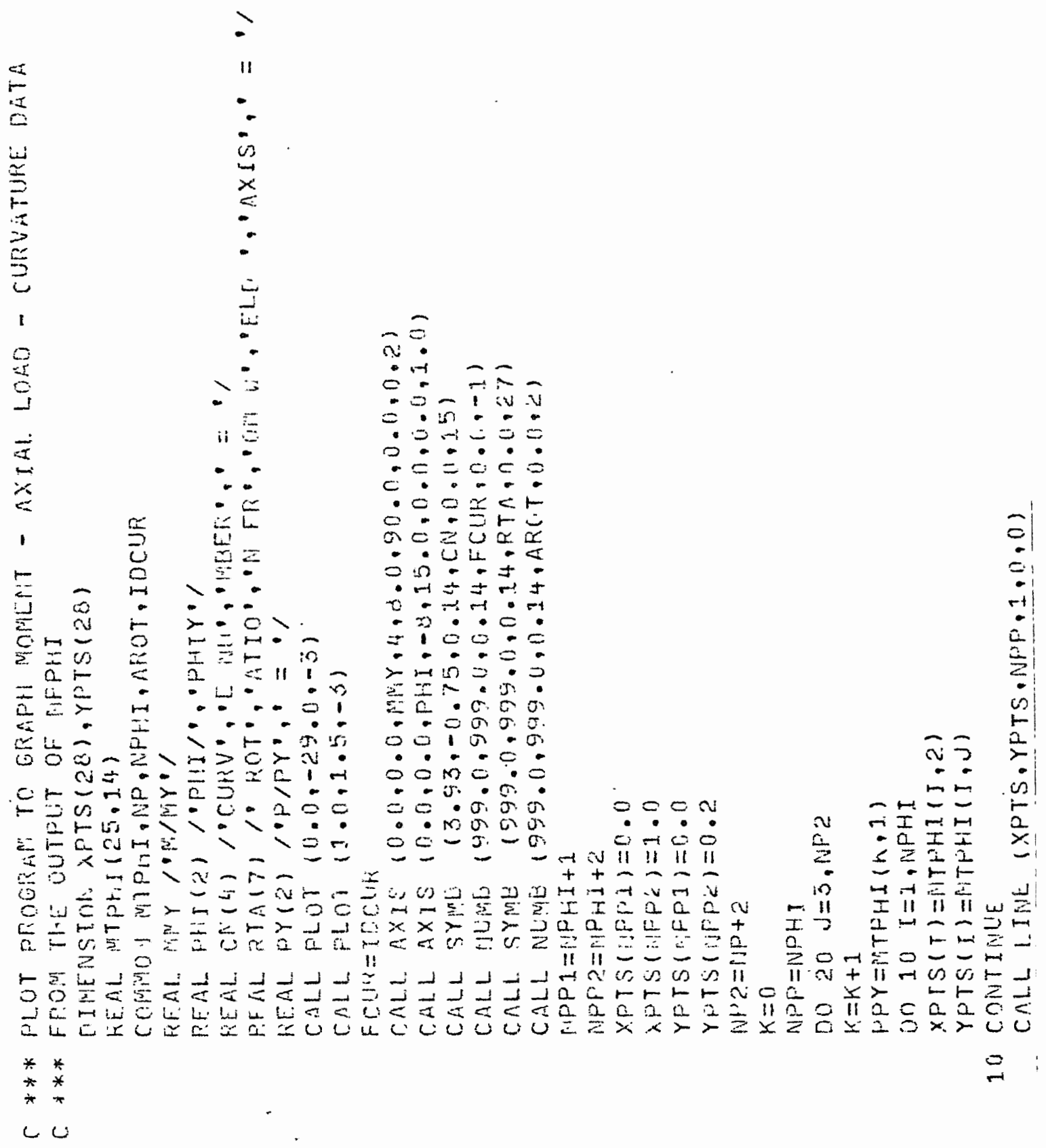

$m$ m 


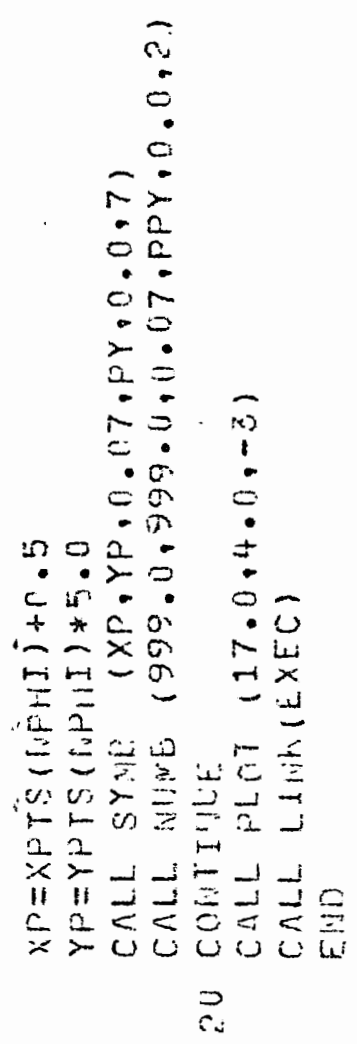

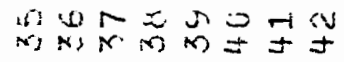

